\title{
The Nature and Evolution of Classical Double Radio Sources from Complete Samples
}

\author{
Katherine M. Blundell, Steve Rawlings and Chris J. Willott \\ University of Oxford, Astrophysics, Keble Road, Oxford, OX1 3RH, U.K. \\ To appear in AJ
}

\begin{abstract}
We present a study of the trends in luminosity, linear size, spectral index, and redshift of classical double radio sources from three complete samples selected at successively fainter low radio-frequency flux-limits. We have been able to decouple the effects of the tight correlation between redshift and luminosity (inherent in any single flux-limited sample) which have hitherto hindered interpretation of the relationships between these four source properties. The major trends found are that (i) spectral indices increase with linear size, (ii) rest-frame spectral indices have a stronger dependence on luminosity than on redshift except at high $(\mathrm{GHz})$ frequencies, and that (iii) the linear sizes are smaller at higher redshifts. We reproduce the observed dependences in a model for radio sources (born throughout cosmic time according to a radio-source birth function) whose lobes are fed with a synchrotron-emitting population from compact hotspots, and which suffer inverse Compton, synchrotron and adiabatic expansion losses. The magnetic energy density within each hotspot is proportional to the jet-power, and synchrotron losses suffered in the hotspot mean that the energy spectrum of the emitting particles fed to the lobes is governed by the jet-power. The axial ratios of radio sources in our model increase as the sources age, and axial ratios are higher in sources with higher jet-power. In simulating the basic observed dependences, we find that there is no need to invoke any systematic change in the environments of these objects with redshift if the consequences of imposing a survey flux-limit on our simulated datasets are properly included in the model. It is also necessary to include appropriate energy loss mechanisms (such as the effects of the cosmic microwave background and feeding the lobes from a compact hotspot) which cause decreasing luminosity through the life of a source. Although our study has broken the luminosity-redshift degeneracy, we present evidence that for such studies there is an unavoidable "youth-redshift degeneracy", even though radio sources are short-lived relative to the age of the Universe; it is imperative to take this into account in studies which seemingly reveal correlations of source properties with redshift such as the "alignment effect".
\end{abstract}

Subject headings: radio continuum: galaxies — galaxies: evolution — galaxies: jets — galaxies: active — quasars: general 


\section{Introduction and scope}

In order to explore the physical mechanisms by which classical double radio sources are governed, observations of samples of such objects are needed to discern the salient relationships between different source properties, such as spectral index or luminosity.

Even observations of a complete sample will inevitably be convolved with the cosmology of the Universe as well as with the consequences of the sample selection process. We first describe (in \$2) how to avoid compounding the latter problem and giving rise to spurious, or hard to interpret, results.

In $\S 3$ we briefly describe the samples we have used in this new analysis, including the new 7C Redshift Survey, which allow us to avoid problems inherent in the sample selection processes. In $\$$ - we describe the principles of how we use these data, the types of objects included in the analyses, and how these principles were put into practice. We then examine in $\$ 5$ the dominant trends to emerge in the various source properties. In $\$ 6$ we present a parameterisation of our results for ease of comparison with previous results. In $\oint \sqrt{7}$ we then review and briefly discuss results from the past which have hinted at the results we find. In order to interpret these results, we develop our model of radio sources in $\S 8$; within this section, we summarise in $\$ 8.1$ basic properties of radio sources which are widely believed to be correct and discuss what may be gleaned about the environments in which radio sources reside in 8.2 . We then consider the expansion of the source and observational constraints on expansion speeds in $\$ 8.3$. A model for the luminosity evolution for a given source is considered in $\$ 8.4$; in this section we present a model for the rôle played by the hotspot in governing the luminosity of radio source lobes. After considering in $\$ .5$ the development of individual radio sources we describe in $\$ 8.6$ how to correctly sample radio sources born at successive cosmic times in order to reproduce simulated versions of the data from our complete samples. In $\$ 8.7$ we present simulated versions of our complete samples using the modelling described in previous sections. In $\S 9$ we discuss what has been learned about the physical mechanisms by which radio sources are governed from our data and simulations, and briefly consider some implications for cosmological studies. We summarise the salient conclusions of our study in $\S 10$.

We assume that $H_{\circ}=50 \mathrm{~km} \mathrm{~s}^{-1} \mathrm{Mpc}^{-1}$ and, except where specifically stated otherwise, that $\Omega_{\mathrm{M}}=$ 1 and $\Omega_{\Lambda}=0$. We use the convention for spectral index $(\alpha)$ that $S_{\nu} \propto \nu^{-\alpha}$, where $S_{\nu}$ is the flux density at frequency $\nu$.

\section{Sampling and selection}

\subsection{The ineluctable biases in complete sam- ples}

The simplest sample-type, referred to here and in companion papers as a 'complete sample' has as its selection criteria only a single flux-limit (determined ultimately by the finite sensitivity of the survey telescope) in a chosen observing band and a chosen sky area. However, for any single flux-limited sample chosen in this way there will be an inevitable and tight correlation between luminosity and redshift. This arises because for a fixed flux-limit, only powerful sources can be detected out to great distances. It is therefore impossible to determine for a single fluxlimited sample whether the dependence of a given source property, such as linear size, is primarily on redshift or on luminosity. Figure 11 shows the coverage of the luminosity-redshift $(P-z)$ plane by particular complete samples selected at different flux limits.

It is clear that just one such sample neither achieves a good range in luminosity out to high redshift nor a good range in redshift at low luminosity. The use of only one sample severely undermines any study pertaining to the evolution of radio sources. Extensive coverage of the luminosity-redshift plane is essential if we are to decouple the luminosity/redshift dependence of a given source property and this is only achievable, given restricted telescope time, using a number of complete samples selected at increasingly fainter flux-limits.

\subsection{The choice of selection frequency}

Samples selected at high frequency $(\gtrsim 1 \mathrm{GHz})$ come with complications: at these frequencies the cores of radio sources can be a significant contribution to the total flux density. Such cores are thought to be increasingly Doppler boosted as the angle between the jet axis and the line-of-sight is decreased (see e.g. Vermeulen \& Cohen 1994). This means that objects at a small viewing angle are preferentially included in the sample, since their total flux measure-

\footnotetext{
${ }^{1}$ For further discussion of the details and subtleties which contribute to this close relationship see $\S 9.3$.
} 


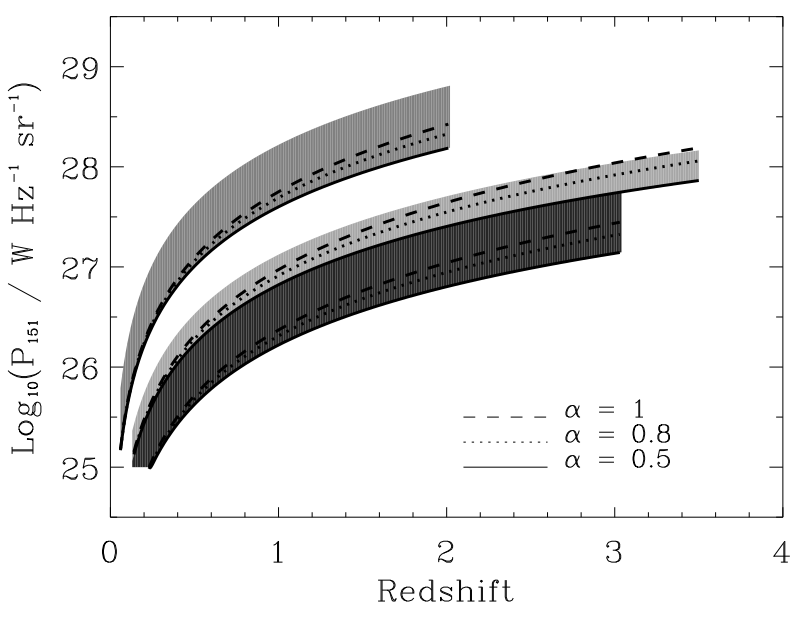

Fig. 1.- A schematic illustration of the coverage of the luminosity (at $151 \mathrm{MHz}$ rest-frame) versus redshift $(P-z)$ plane from a single sample selecting all sources brighter than $12 \mathrm{Jy}$ in a certain sky area (coloured mid-grey), a single sample of sources with flux densities between 2 and 4 Jy (coloured lightgrey) and a single sample which has selected sources brighter than $0.5 \mathrm{Jy}$ in a much smaller area of sky (coloured dark-grey). Each coloured region contains a similar number of sources. The solid black lines in each case correspond to the minimum luminosity which a source at that redshift may have, and yet still be above the minimum flux-limit of the sample assuming source spectral indices are 0.5 ; other linestyles indicate the influence of other spectral indices on the shape of the flux-limit on the $P-z$ plane. The upper edge of the mid-grey region arises because of a maximum observed luminosity in radio sources. The upper-edge of the dark-grey region arises because in its small sky area the probability of finding more luminous sources is low.

ment is promoted over the sample flux-limit because of their orientation, rather than their intrinsic power. In addition to having lower power in extended components, these objects will have shorter projected angular sizes. Moreover, the $\mathrm{GHz}$ part of the spectrum of radio sources is reduced due to synchrotron losses as the source radiates, adiabatic expansion losses as the source grows and expands and, with increasing importance with increasing redshift, due to inverse Compton losses from the equivalent magnetic field of the cosmic microwave background radiation. This is further compounded because of the increasing difference between emitted frequency and observed frequency as redshift increases. Thus observing at a high frequency $(\gtrsim 1 \mathrm{GHz})$ will result in a vast loss in the number of high redshift sources which make it above the sample limit, since inverse Compton losses increase dramatically with redshift [the equivalent magnetic field due to the cosmic microwave background (CMB) is given by $0.318(1+z)^{2} \mathrm{nT}$ ]. Inverse Compton losses have been invoked to explain the X-ray flux from the lobes of the nearby radio galaxy Fornax A (Kaneda et al. 1995) and also as an explanation of the high Xray luminosity of at least some distant powerful radio galaxies (Brunetti, Setti and Comastri 1997). Sources with large linear sizes at intermediate and high redshifts are most unlikely to be found in high frequency surveys since larger sources tend to have steeper spectral indices (see $\$ 9.4$ ). [Thus high frequency surveys are a poor probe of evolution in the high redshift universe, except for the Doppler boosted population.]

Surveys carried out at lower frequencies (a decade lower $\sim 0.1 \mathrm{GHz}$ ) detect emission largely from that part of the spectrum which is least subject to synchrotron/inverse Compton losses and orientation biases. They thus inform on the intrinsic energy spectrum injected into the radio lobes in a way which is less affected by the history of that particular source. There is thus a strong argument for using complete samples detected at such frequencies. However, in making a radio survey at arbitrarily low frequency one encounters different problems: there are three mechanisms by which a survey conducted at $\sim 10$ $\mathrm{MHz}$ would fail to detect sources which surveys at higher frequency would detect. One of these mechanisms is synchrotron self-absorption; this arises when the intensity of synchrotron radiation within a source becomes sufficiently high that re-absorption of the radiation by the synchrotron emitting particles is important. Another mechanism is free-free absorption: absorption of the synchrotron radiation by thermal material intervening along the line-of-sight, possibly in the immediate vicinity of the synchrotron source - e.g. by its own cluster environment; an example of this mechanism in 3C 295 is discussed by Taylor \& Perley (1992).

The third mechanism arises because of a lowenergy cut-off to the relativistic particles which give rise to the synchrotron emission. Arguments that there is a departure from a power-law in the electron- 
energy distribution at low energies were advanced by Leahy, Muxlow \& Stephens (1989) who made observations of $3 \mathrm{C}$ radio sources at $151 \mathrm{MHz}$ with MERLIN and at $1.5 \mathrm{GHz}$ with the VLA and found spectral flattening in the hotspots of three of the powerful sources at low frequency.

An extreme example of sources which are rarely detected in low-frequency surveys are the Giga Hertz Peaked Spectrum (GPS) population [O'Dea, Baum \& Stanghellini (1991)]. It is the turnover in the spectrum of these sources which gives rise to the absence of these sources in low-frequency surveys, and their presence in high-frequency surveys.

The lower the frequency of the detection survey, the greater is the chance of losing small sources via one of these mechanisms. However, for a given turnover frequency, this chance decreases with increasing redshift. A sample selected at a fairly low frequency (such as tens of $\mathrm{MHz}$ ) might miss small low redshift sources which would be detected at higher redshift because the observing frequency is likely to be less than the turnover frequency. In order to quantify the significance of this effect in samples selected at a frequency of $38 \mathrm{MHz}$, we considered that complete sample which is a subset of the $3 \mathrm{C}$ sample (described in $\$ 3.1)$ defined by declination, $\delta>60^{\circ}$. We then considered which of the sources in this sample of 29 objects would not have been included in a survey selected in the same sky area at the lower frequency of $38 \mathrm{MHz}$, due to spectral turnover.

The limiting flux density of the $8 \mathrm{C}$ survey carried out at $38 \mathrm{MHz}$ Rees (1990); catalogue revised by Hales et al. (1995) is 1 Jy over most of the survey area. We set a threshold of $25 \mathrm{Jy}$ for our $38-\mathrm{MHz}$ selected sample since this is the flux density which an object on the $3 \mathrm{C}$ flux-limit at $178 \mathrm{MHz}$ would have at $38 \mathrm{MHz}$ if it had a spectral index of 0.6 - i.e. a spectral index which can plausibly arise without any recourse to one of the spectral turnover mechanisms discussed above.

Three bona fide sources from a sample of 29 objects would be lost because of spectral turnover; relevant properties of these objects are shown in Table 1. Thus the deficit of small objects (none of which are at high redshift) missing from a sample selected at 38 $\mathrm{MHz}$ rather than a somewhat higher frequency e.g. $178 \mathrm{MHz}$ is at the $\sim 10 \%$ level. We therefore note that stronger linear size evolution might in principle be found from surveys at $38 \mathrm{MHz}$ because of the absence of small (low redshift) sources.
Observing at a higher frequency will tend to detect a higher number of small low-redshift sources which have a spectral turnover, since a higher observing frequency is more likely to be above typical turnover frequencies. Even with this, we suggest that a high frequency like $408 \mathrm{MHz}$ might measure spuriously high linear size evolution, [since a survey at $408 \mathrm{MHz}$ selects objects at $z \sim 2$ at rest-frame $1 \mathrm{GHz}]$, since large and small low redshift sources will still make it into the sample but the only high redshift sources which make it into the sample are very short ones which are too young to have suffered catastrophic inverse Compton losses to their lobes.

To investigate the effects of increasing the survey frequency on the selection process, we considered the differences between the $6 \mathrm{C}$ sample selected at 151 $\mathrm{MHz}$ (described in \$3.2) and a sample selected over the same region of sky but at $408 \mathrm{MHz}$. We were able to do this using the sample of Allington-Smith (1982) which is roughly the same region of sky as the $6 \mathrm{C}$ sample but selected at $408 \mathrm{MHz}$ to be brighter than 1 Jy as measured in the Bologna (B2) survey. Those sources which are only selected at $408 \mathrm{MHz}$ in this way are listed in Table 2. We have redshift information for all but one $(\mathrm{B} 20927+35)$ of these objects hence we could overlay the points from this sample on our various plots of the four different source properties (luminosity, spectral index, redshift and linear size; an example is presented in Figure 8). We found that while selection of this sample at the higher frequency does result in the inclusion of more objects which tend to have fairly small projected linear sizes, these are over a range in redshift and we discern no redshift systematic bias implied by the properties of the sources included in the sample selected at 408 $\mathrm{MHz}$ rather than $151 \mathrm{MHz}$, at these flux-limits.

\subsection{The use of one selection frequency}

Neeser et al. (1995) pointed out that comparing samples with very different selection frequencies will find a spurious evolution in linear size, if a faint high frequency (e.g. $\sim 1 \mathrm{GHz}$ ) sample with the biases described in $\$ 2.2$ were to be compared with a bright low frequency (e.g. $0.1 \mathrm{GHz}$ ) sample without these biases. A study of the evolution of radio sources based on samples selected at different frequencies is not helpful because it amounts to a comparison between the same types of objects at different parts of their radio spectra. 


\subsection{Source type}

It should be clear that samples which are incomplete or inhomogeneous should not be used for a study pertaining to evolutionary properties because of the subtle and unsubtle biases which will be present. Even "well-defined" samples are not without their problems. For example, past studies [e.g., Barthe] $\&$ Tiley (1988) have used samples comprised only of steep spectrum quasars. The motivation for such an approach is understandable: the spectroscopy is relatively easy hence good coverage out to high redshift may be achieved. However, if we believe the unification by orientation model [Scheuer (1987)]; Barthe] (1989)] at some level (and thus that for quasars the jet axis is at a small angle to the line of sight), then the projected angular sizes which we measure will be heavily foreshortened. One cannot begin to deproject the sizes unless one has very large numbers of sources and a good idea what the mean and spread in the transition angle are. This problem is potentially compounded if quasars are used which are not from complete samples but have optical selection criteria as well, since this might preferentially bias towards boosted objects which are pointing towards us (see Willott et al. 1998a).

In principle therefore, studies which have used only radio galaxies, which are presumed to lie closer to the plane of the sky and therefore will have a smaller difference between the projected size we measure and the deprojected size we would wish to measure, might be a more reliable indicator of the true dependences. In fact we chose to use all radio galaxies and quasars in our samples which fulfilled the criteria discussed in $\oint$. Such a selection criterion should ensure a random source orientation, allowing statistical deprojection of the linear size information.

\subsection{Spectroscopy}

Many previous studies of samples of radio sources have required the use of redshift estimators for a significant fraction of the sample (e.g. Dunlop \& Peacock 1990). The most common estimator employs the $K$-band $(2.2 \mu \mathrm{m})$ magnitude. Use of this estimator is motivated by the small spread in the near-infrared Hubble Diagram ( $K-z$ relation) for radio galaxies in the 3C sample (Lilly \& Longair 1984; Best, Longair and Röttgering 1998). It is now known that simple extrapolation of the $3 \mathrm{C}$ relation to higher redshifts and/or to fainter radio samples (which contain fewer radio luminous sources at any given redshift) leads to both large uncertainties in the estimated redshifts, and to systematic biases. At redshifts of $z>2$ the $K$-magnitude becomes contaminated by luminous emission lines, and other sources of non-stellar radiation [Eales \& Rawlings (1993, 1996); Simpson, Rawlings \& Lacy (1998)]. This is at least partially responsible for a scatter of 3 magnitudes in the $K$ $z$ diagram at $z>2$, and the little, if any, correlation between the variables (Eales et al. 1993). This large scatter may also be influenced by a genuine increase in the spread of stellar luminosities at early cosmic epochs. Comparison of the $K-z$ relations of $3 \mathrm{C}$ and $6 \mathrm{C}$ samples (Eales et al. 1997) reveals a significant difference in the sense that $3 \mathrm{C}$ radio galaxies are about one magnitude brighter than the $6 \mathrm{C}$ galaxies at $z \approx 1$. Further studies (Best et al. 1998; Roche, Eales \& Rawlings 1998) suggest that this effect is caused by a (positive) correlation between radio luminosity and the stellar luminosity of the host galaxy which is not yet well understood. To summarise, although it may now be possible to construct more reliable singlecolour redshift estimators using $K$-band imaging of the combined $3 \mathrm{C} / 6 \mathrm{C} / 7 \mathrm{C}$ dataset, the use of almost exclusively spectroscopic redshifts has been an essential ingredient of the work described in this paper. In the very few cases where we have been unable to secure spectroscopic redshifts $(\S 3.2$ and $\S 3.3)$, we have estimated redshifts by obtaining multi-colour (optical and near-infrared) information and fitting spectral energy distributions (SEDs) with template galaxy spectra (e.g. Dunlop \& Peacock 1993). This method is likely to be robust whenever non-stellar contributions to the SED can be safely neglected. Evidence that this is the case comes from the lack of any detectable emission lines in both optical and near-infrared spectroscopy of the relevant objects.

\section{Samples used}

\section{1. $3 \mathrm{C}$}

The complete sample is that of the revised 3CR catalogue of Laing, Riley \& Longair (1983), which contains 173 objects in an area of sky of $4.239 \mathrm{sr}$ with $\delta \geq 10^{\circ}$ and $|b| \geq 10^{\circ}$ each with a flux den- 
sity 2 greater than 10.9 Jy at $178 \mathrm{MHz}$. We excluded 3C 231 (M 82) since its radio emission is due to starformation (Condon et al. 1990) rather than an active galactic nucleus. We also excluded another two objects, 3C 345 and 3C 454.3, because their emission at $178 \mathrm{MHz}$ only exceeds the flux-limit because of Doppler boosting of their core emission. The final redshift of this sample, for 4C 13.66, was obtained by Ra vlings et al. (1996b); the penultimate redshift, for $4 \mathrm{C} 74.16$, is 0.568 (R.A. Laing, priv. comm.). The spectroscopic redshift completeness for this sample is thus $100 \%$.

\section{2. $6 \mathrm{C}$}

The complete sample we used is based on Eales' (1985) sample, but re-selected from the final versions of parts II and VI of the $6 \mathrm{C}$ survey at $151 \mathrm{MHz}$ [Hales et al. (1988) and Hales et al. (1993) respectively]. This revised sample is for objects with $2.0 \leq S_{151}<3.93$ Jy (where $S_{151}$ is the flux density in Jy at $151 \mathrm{MHz}$ ), with $082030<\mathrm{RA}(\mathrm{B} 1950)<130130$ and also with $+340100<\delta(\mathrm{B} 1950)<+400000$. The sky coverage of this sample is thus $0.102 \mathrm{sr}$. Details of the revised sample are in Blundell et al. (1998b) and redshifts are in Rawlings et al. (in prep.). Structural classifications and angular sizes were re-measured from maps by Law-Green et al. (1995), Naundorf et al. (1992), Naundorf (1992) and Tansley (1997). The revised complete sample would consist of 59 objects but one $(6 \mathrm{C} 1036+3616)$ is formally excluded for a reason which is not statistically biassed, namely because a bright star lies too close to its optical identification to measure the redshift of the radio source. Only one redshift for this final sample of 58 sources is not spectroscopic giving $98 \%$ spectroscopic redshift completeness for the $6 \mathrm{C}$ sample.

\section{3. $\quad 7 \mathrm{C}$}

The $7 \mathrm{C}$ survey was carried out with the Cambridge Low Frequency Synthesis Telescope (CLFST) at 151 $\mathrm{MHz}$ McGilchrist et al. (1990)] at a resolution of $70 \times 70 \operatorname{cosec}(D e c) \operatorname{arcsec}^{2}$. In this paper we use the $7 \mathrm{C}-\mathrm{I}$ and $7 \mathrm{C}-\mathrm{II}$ parts of the $7 \mathrm{C}$ Redshift Survey, which were chosen by Rossitter (1987) to over-

\footnotetext{
${ }^{2}$ For the analysis described in this paper, flux densities quoted by Laing et al. (1983) for all objects in this sample were multiplied by 1.09 to put them on the same flux scale as Baars et al. (1977)

${ }^{3}$ This roughly corresponds to a flux-limit of $12 \mathrm{Jy}$ at $151 \mathrm{MHz}$.
}

lap with the 5C6 and 5C7 fields of Pearson \& Kus (1978), respectively. The 7C 151-MHz data were subsequently re-analysed (and in the case of the $5 \mathrm{C} 6$ field, re-observed) by Julia Riley. VLA data, where available, were retrieved from the archive (by kind permission of Barry Clark), or were recently obtained by us: full details of all these radio observations and the sample selection are described by Blundell et al. (1998b). The $7 \mathrm{C}-\mathrm{I}$ region covers an area of 0.0061 sr and contains 37 sources with $S_{151} \geq 0.51 \mathrm{Jy}$. The $7 \mathrm{C}-\mathrm{II}$ region covers an area of $0.0069 \mathrm{sr}$ and contains 40 sources with $S_{151} \geq 0.48 \mathrm{Jy}$. All the sources in the final sample have now been mapped with the VLA giving high-resolution radio maps. We used these maps to deduce the structural information and angular size of each object in the 7C sample. Spectroscopic redshifts have been obtained for 71 members of the $7 \mathrm{C}$ complete sample, thus spectroscopic redshift completeness is $92 \%$ (Willott et al. in prep.). For the remaining objects, their redshifts are estimated from 4 or 5 optical/near-infra red magnitudes as described in Rawlings et al. (in prep.). Only one source in the small sky area of the $7 \mathrm{C}$ sample $(3 \mathrm{C} 200)$ is common to the $3 \mathrm{C}$ sample, and this source is just included as a member of $3 \mathrm{C}$ for the purposes of the analysis presented in this paper.

We excluded one object, 5C7.230 (also known as $7 \mathrm{CB} 082134.3+244829$ ), from the sample because its emission at $151 \mathrm{MHz}$ only exceeds the flux-limit because of Doppler boosting of its core emission (Blundell et al. 1998b).

\section{Classifications \& measurement techniques}

\subsection{Source structure...}

Neeser et al. (1995) demonstrated the perils of trying to analyse the changes in maximum angular extent of the 'edge-darkened' FR I-class Fanaroff \& Riley (1974) radio sources with increasing redshift and attributed the inclusion of such objects to the extremely strong linear size evolution with redshift which has been reported in the past (e.g. Oort, Katgert \& Windhorst (1987)]). To avoid spurious correlations and to focus our analysis on the evolution of one physically distinct class of object out to very high redshift, i.e., to study the same physical phenomenon at different redshifts in order to compare like with like, we investigated the radio spectra and structure of the objects in our samples. One analysis which is part of this paper - the linear size evolution of classical double 
radio sources - amounts to considering how the separation of working surfaces at the ends of oppositely directed radio jets depends on luminosity and/or redshift. For this reason we excluded from our analysis radio sources of the FR I structural type. We regard objects of this morphological type as the inevitable manifestation of low jet-power objects, where the jetpower is insufficient to form a supersonic shock, given its environment, where the jet impinges on the intergalactic medium [see e.g. Falle (1991)]. The 6C sample and $7 \mathrm{C}$ sample selected to be a factor of 6 and a factor of nearly 25 respectively fainter than $3 \mathrm{C}$, in fact contain relatively few objects of FR I structure. This arises because in the relatively small sky areas of $6 \mathrm{C}$ and 7C few nearby bright but low-luminosity objects are found. Note that luminosity is not a fundamental quantity in these objects in the way that the power transported by the jet is. Distant low-luminosity objects which are found will not be the exact counterparts of low-redshift objects of the same luminosity: they must have higher jet power to compensate for the fact that for an object of redshift $z$, inverse Compton losses increase as $(1+z)^{4}$. Put another way, an object with a given jet power observed in an environment with a given density profile at high redshift will have a much lower luminosity at, say, $80 \%$ of the way through its life than a lower redshift object with otherwise the same parameters at the same point. [Note that this is not an observational error: the selection is made at a sufficiently low frequency and resolution that there is no question of missing sources because of undersampling smooth extended flux.]

For this reason we do not base the criteria of which objects should be included in our analysis on their luminosity, but on their structure.

How then to identify whether a given source is type FR I or FR II? We deemed that an object was a classical double (FR II) if at least one hotspot was at the leading edge of a working surface. In practice this meant a bright, compact feature with a sharp discontinuity in surface brightness was at the leading edge of emission expanding outwards from the core or location of the optical identification. We did not require that this was the case on both sides of the core [see e.g., 5C6.62 also known as 7C B021029.7+325402 (Blundell et al. 1998b)] to allow for the fact that although a working surface can exist, it can have a slightly weird appearance due to e.g., Doppler suppression, but the presence of one such hotspot is a good indicator that in the environment in which a given source is located, the jet-power is sufficiently high that a classical double radio source will be manifested.

In some objects there was clear evidence of emission beyond a possible working surface, which had decreasing surface brightness with increasing distance from the core. We considered these to be a somewhat different physical phenomenon from the 'working surface + backflow towards the core' type object, and called them FR Is and excluded them from our analyses. For those intermediate cases where we could not be totally sure that an object should be assigned to the FR II category, we considered by examination of their integrated radio spectra (see \$4.2) whether there was any evidence of trailing outflow with increasing distance from the core, as might be evidenced by a greater flux from a survey measurement (e.g., from VLA D-array or single dish measurements) than from our higher resolution (VLA A- or B-array) measurements. Thus, objects which do not apparently contain any clearly defined working surface, but in which we believe all the flux is contained within a well-defined measureable extent, are classed as having defined angular sizes (DAs). We believe that most, if not all, the angular sizes measured for these objects do correspond to the separation of working surfaces at the ends of oppositely directed radio jets, though we cannot eliminate the possibility that some might be FR Is.

Our angular size measurements were our best attempt at summing the separations of the two working surfaces from the core; in the absence of a radio core we took our best estimate of the separations of the working surfaces from one another.

\section{2. $\quad \ldots$ and Spectra}

Fitting of the radio spectra for the members of all three samples was made possible by consideration of data from many radio surveys 7 , and in the case of the 7C sample in combination with data from our own VLA maps as tabulated in Blundell et al. (1998b).

We fitted the flux density data using a Bayesian polynomial regression analysis which assessed the posterior probability density function (PDF) for the required order of a polynomial fit (e.g. Sivia 1996); we

\footnotetext{
${ }^{4}$ In addition to the original survey data at 151 or $178 \mathrm{MHz}$, we obtained from the literature flux densities at $365 \mathrm{MHz}, 408$ $\mathrm{MHz}, 1.4 \mathrm{GHz}$ and $4.86 \mathrm{GHz}$, and in the case of $3 \mathrm{C}$ we also obtained flux densities at 10,22, 38 and $85 \mathrm{MHz}$, chiefly from Laing \& Peacock (1980).
} 
chose $x=\log _{10}(\nu / \mathrm{MHz})$ as the independent variable and expanded the dependent variable $y=\log _{10} S_{\nu}=$ $\sum_{r=0}^{N} a_{r} x^{r}$. In a number of cases the peak in the PDF led us to prefer a first-order polynomial, and the gradient of the fit $\left(a_{1}\right)$ was multiplied by -1 to give the spectral index. In many cases the PDF led us to prefer a second-order fit, implying significant curvature in the radio spectrum: in these cases we calculated the curvature $\beta=-2 a_{2}$, and hence the spectral index at (rest frame) $151 \mathrm{MHz}$ which equals $-a_{1}+\beta \log _{10}(151 /(1 .+z))$. In no case was a higher order fit preferred.

With a fitted functional form for the spectrum of each object, we interpolated (in the case of 3C) or extrapolated (in the case of $6 \mathrm{C}$ and $7 \mathrm{C}$ ) to derive the flux densities at rest-frame $151 \mathrm{MHz}$ and together with the redshifts we derived the luminosities in $\mathrm{W} \mathrm{Hz}^{-1} \mathrm{sr}^{-1}$ in a number of different assumed cosmologies, according to the formulae presented in Carroll, Press \& Turner (1992).

\section{Results}

We derived all plots in the following three assumed cosmologies: (1) $\Omega_{\mathrm{M}}=0$ and $\Omega_{\Lambda}=0$, (2) $\Omega_{\mathrm{M}}=1$ and $\Omega_{\Lambda}=0$ and (3) $\Omega_{\mathrm{M}}=0.1$ and $\Omega_{\Lambda}=0.9$; similar dependences were seen in each case. We also considered separately just the class of certain FR IIs and also the combined set of FR IIs and DAs (see \$4.1). No significant difference was found. The plots we present here are for the combined set of FR IIs and DAs and, except for Figure 3, an assumed cosmology with $\Omega_{\mathrm{M}}=1$ and $\Omega_{\Lambda}=0$ but the statistical analysis presents the numbers for all three cosmologies we considered (see \$5.2).

\subsection{Plots of source dependences}

Figure 2 shows the luminosities vs projected linear sizes (the $P-D$ plane) of the radio sources. No striking dependence of the luminosity of an object on its linear size is seen, though there is a deficit of small, low-power objects in the lower-left quadrant of the $P-D$ plane, and also of large, high-power objects in the upper-right quadrant of the $P-D$ plane. It can be seen that the region most densely occupied by the solid circles (corresponding to objects with $z \gtrsim 2$ ) is displaced to the left [smaller projected linear sizes] of the region occupied by the main density of plus signs (corresponding to objects with $z<0.8$ ), indicating a distinct anti-correlation of linear-size with redshift.

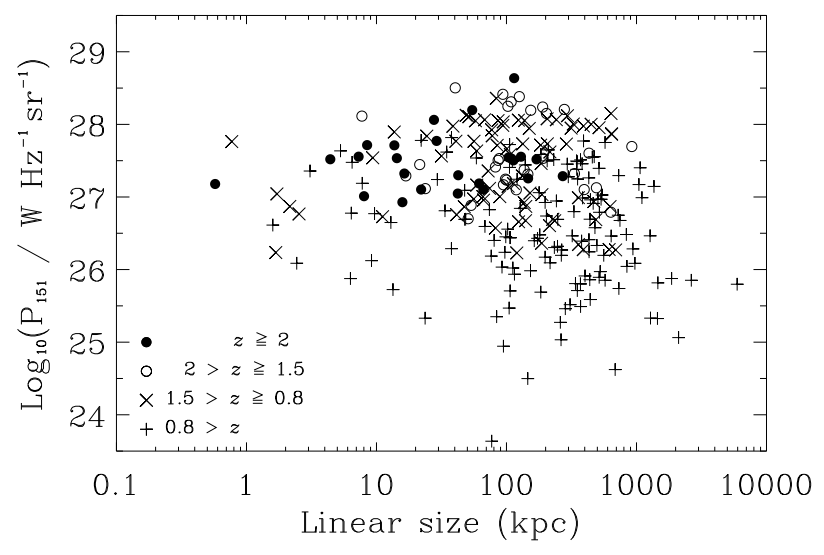

Fig. 2.- This graph shows for all members of the complete samples included in our analysis their luminosities plotted against their projected linear sizes. These properties are calculated assuming $\Omega_{\mathrm{M}}=1$ and $\Omega_{\Lambda}=0$. Four different redshift bins are indicated by symbol type.

Figure 3 shows more directly that sources at higher redshift tend to have shorter projected linear sizes. This trend is seen throughout the redshift range, and is seen in each of our three considered cosmologies. The symbol type in this figure delimits the luminosity bin an object is in. In the middle plot where the assumed cosmology is $\Omega_{\mathrm{M}}=1$ and $\Omega_{\Lambda}=0$, the most powerful sources are not as powerful as in the cosmologies with low $\Omega_{\mathrm{M}}$. This plot demonstrates that in breaking the degeneracy between luminosity and redshift, above a redshift of 2 the tendency for the sources to be smaller seems to be independent of the fact that the majority of these sources are not in the highest power bin.

The operational definition of spectral index used in the past has been to approximate the radio spectrum of a source by a single power law and to derive $\alpha$ as $-\partial \log S_{\nu} / \partial \log \nu$, invariably in the observed frame, using flux density measurements at two frequencies. When this usage of spectral index is employed, there is a significant spectral index dependence on redshift (see Figure 4) which has been exploited (Chambers, Miley \& van Breugel 1990; Blundell et al 1998a) to find the most distant radio galaxies. This precise technique was used to find the most distant radio galaxy currently known (Rawlings et al. 1996a). Once the curvature and redshifting of the spectra (" $K$ - 

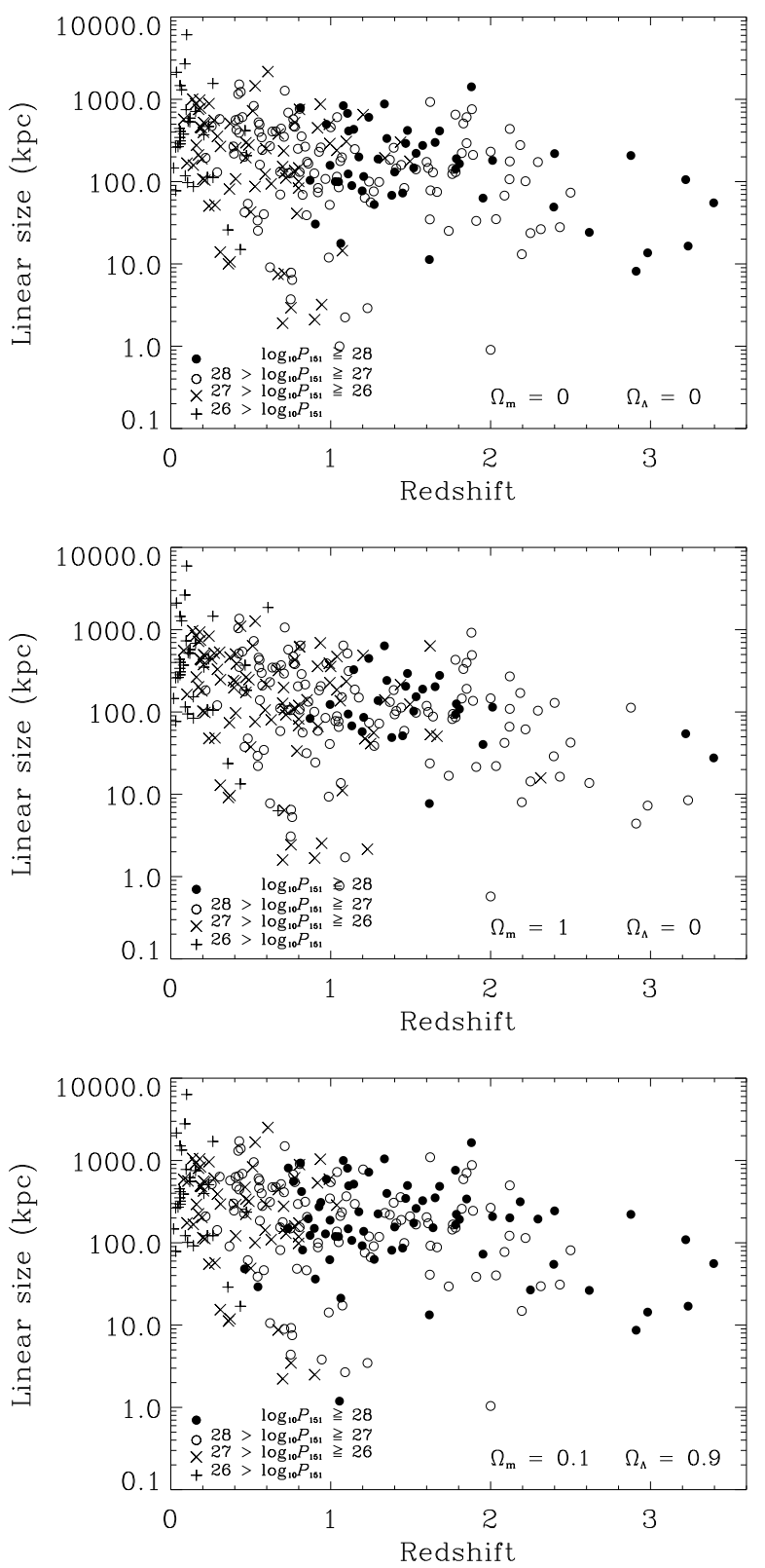

Fig. 3.- These graphs show for all members of the complete samples included in our analysis their projected linear sizes against their redshifts, with these properties calculated for three different assumed cosmologies. The luminosity bin an object falls into is denoted by symbol, with the same threshold values in each case.

correction") are taken account of, this dependence of
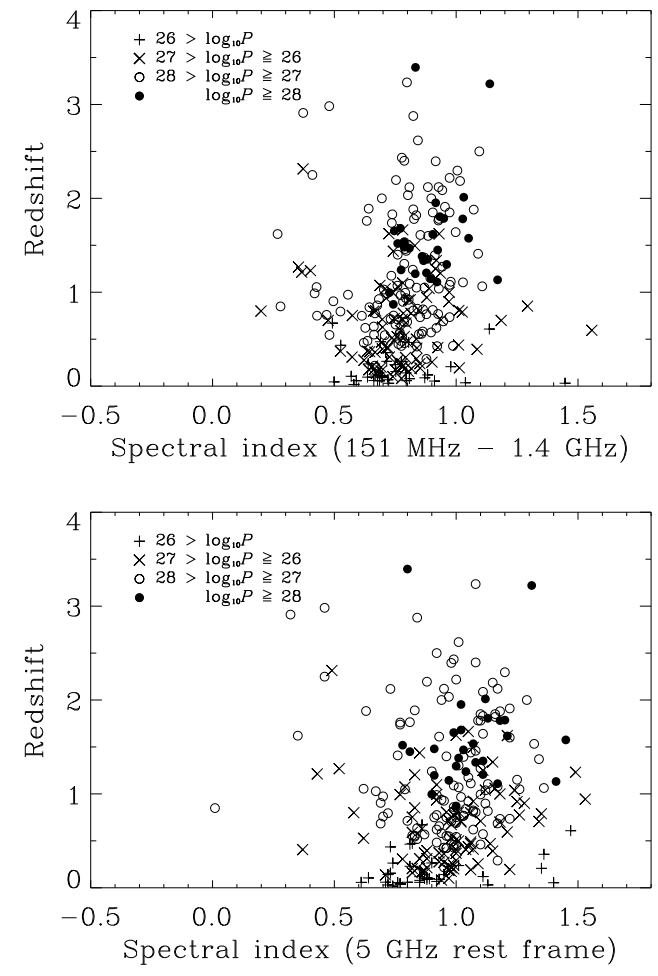

Fig. 4.- These graphs show for all members of the complete samples included in our analysis redshift against spectral index measured in two different ways. The top panel shows the spectral indices before fitting for spectral curvature and $K$-correction and assuming a single power-law between $151 \mathrm{MHz}$ and $1.4 \mathrm{GHz}$ while the lower panel shows spectral indices evaluated at $5 \mathrm{GHz}$ in the rest frame of each source. Source luminosities are indicated by symbol-type.

spectral index on redshift is dramatically different, in a way which depends on the frequency at which the spectral index is evaluated. When the spectral index is evaluated at $5 \mathrm{GHz}$ rest-frame, then a rather strong dependence on redshift is seen (see Figure (1). This arises because the $\mathrm{GHz}$ part of the spectrum is where inverse Compton losses (as well as other loss mechanisms) are most manifest. If spectral indices are evaluated in the rest frame at a frequency of a few hundred $\mathrm{MHz}$, this strong dependence on redshift diminishes (see Figure 5).

Figure 6 shows a plot of the luminosity of each object against its spectral index, each evaluated at rest-frame $151 \mathrm{MHz}$. There is a clear trend for the 

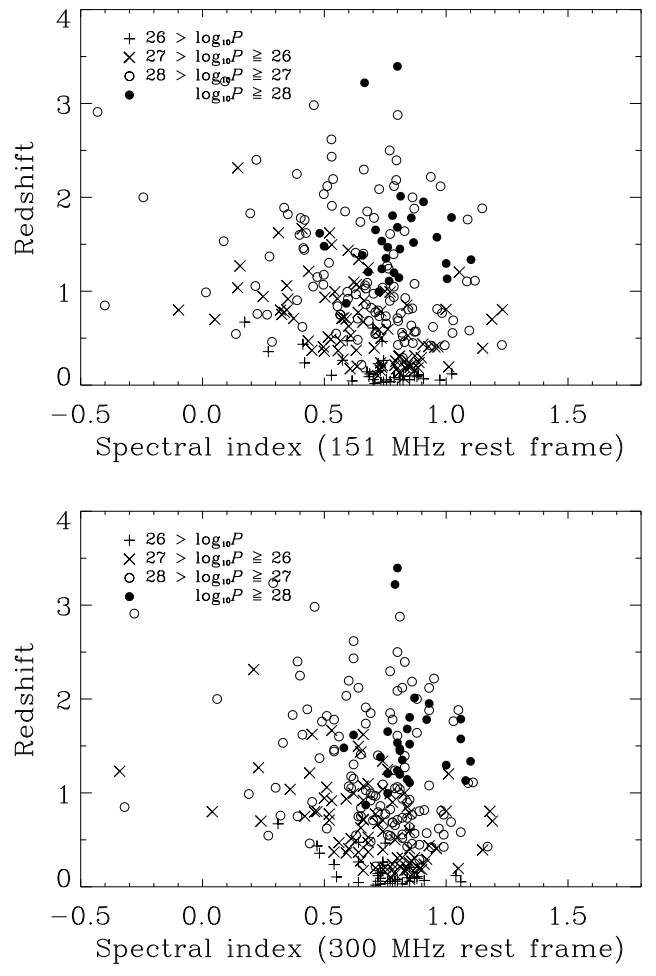

Fig. 5.- These graphs show for all members of the complete samples included in our analysis their redshifts against spectral indices measured in two different ways, in both cases fitting for spectral curvature and $K$-correction. The upper panel has the spectral indices evaluated at $151 \mathrm{MHz}$ in the rest frame while the lower panel shows the spectral indices evaluated at $300 \mathrm{MHz}$ in the rest frame of each source. Source luminosities are indicated by symbol-type.

most powerful objects to have the highest spectral indices.

At a frequency as low as rest-frame $151 \mathrm{MHz}$, the spectral index is informing us on the energy index of the synchrotron particles as injected into the lobes. We hereafter refer to this power-law exponent of the energy distribution of particles injected into the lobes as the 'injection index', reserving the term 'acceleration index' for the exponent of the energy distribution to which particles in the hotspot may accelerated [and described by a model such as diffusive shock acceleration e.g. Bell (1987)]. We explore in $\$ 9.2$ how this injection index may depend in a simple way on the intrinsic jet-power of an object and hence how such

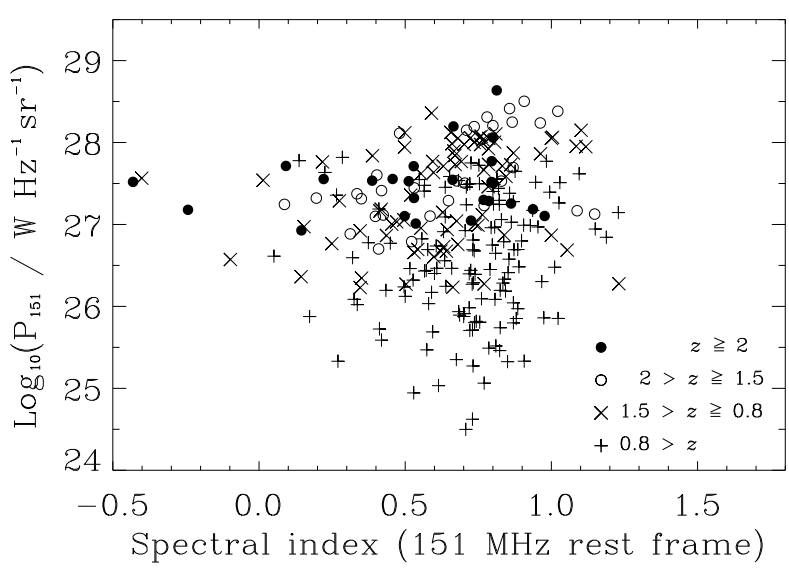

Fig. 6.- This graph shows for all members of the complete samples included in our analysis their luminosities plotted against their spectral indices each evaluated at rest-frame $151 \mathrm{MHz}$. The redshift bins are indicated by symbol type.

an injection-index-luminosity dependence can arise.

A neat manifestation of the $P-\alpha$ correlation is seen in the luminosity-redshift plane. Figure 7 shows lines which trace the flux-limits for $3 \mathrm{C}, 6 \mathrm{C}$ and $7 \mathrm{C}$, i.e. 12, 2 and 0.5 Jy respectively. The solid black lines in each case correspond to the minimum luminosity which a source at a given redshift may have, and yet still be included in a given sample if its spectral index is 0.5 ; other line-styles indicate the influence of other spectral indices on the shape of the flux-limit on the $P-z$ plane. It can be seen that those $3 \mathrm{C}$ sources with luminosities above $\sim 10^{27.5} \mathrm{~W} \mathrm{~Hz}^{-1} \mathrm{sr}^{-1}$ all avoid the region between the flux-limit line for a spectral index of 0.5 and that for a spectral index of 0.8 . For those $3 \mathrm{C}$ sources which have rather lower luminosities, it is impossible to distinguish any preferred flux-limit line. In this lower luminosity regime the fainter samples do not show any such avoidance of the flux-limit line given by a spectral index of 0.5 .

Inspection of the $D-\alpha$ plane depicted in Figure 8 shows a correlation of projected linear size with spectral index, although it also suggests that sources with even quite large linear sizes may not necessarily have very steep spectra at rest-frame $151 \mathrm{MHz}$. It is possible that some of these spectra are flattened by freefree absorption from intervening material. Taylor \& Perley (1992) present a study of 3C295 which has a 

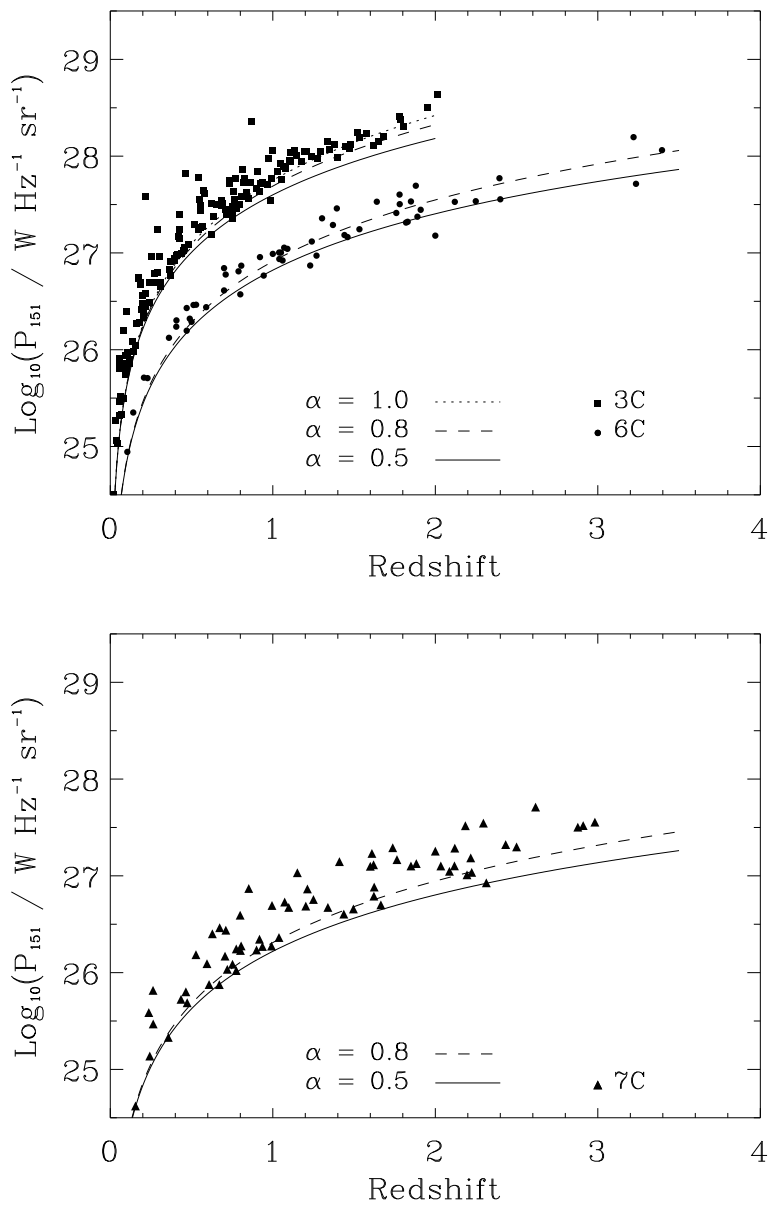

Fig. 7.- These graphs show for all members of the complete samples included in our analysis their luminosities at rest-frame $151 \mathrm{MHz}$ against redshifts. The top panel shows the $P-z$ plane for the $3 \mathrm{C}$ and $6 \mathrm{C}$ samples; the lower panel shows the $P-z$ plane for the $7 \mathrm{C}$ sample.

(complex) turnover in the spectrum below $100 \mathrm{MHz}$; they attribute this to thermal absorption from multicomponent ISM gas at the cluster.

\subsection{Disentangling the different dependences: statistics}

We display in Tables 3 and 7 the results of the statistical analysis using the 4-way Spearman partialrank correlation coefficient described by Macklin (1982), to indicate the true dependences of the different source properties. In these Tables, $r_{a b \mid c d}$ is the corre-

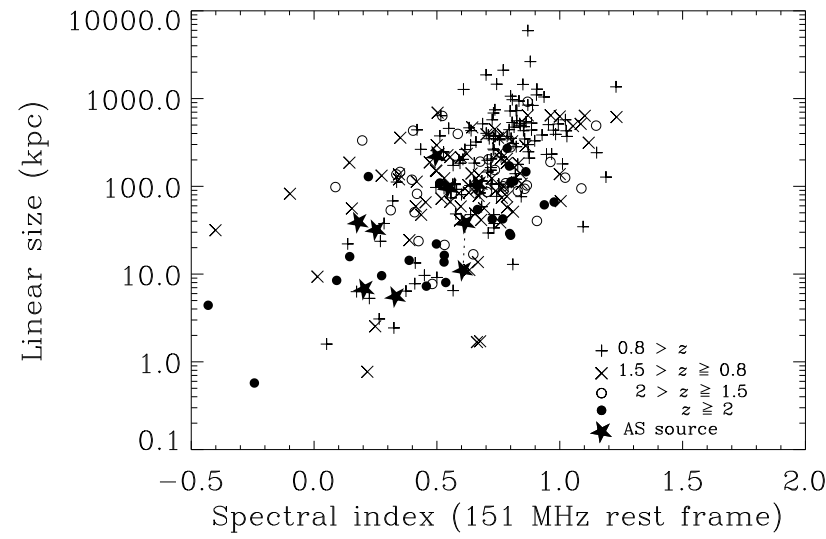

Fig. 8.- This is a plot of projected linear size against spectral index evaluated at $151 \mathrm{MHz}$ rest-frame for all members of the complete samples. We also overlay those sources which would have been members of the $6 \mathrm{C}$ complete sample had the selection frequency been $408 \mathrm{MHz}$ [taken from Allington-Smith (1982); see $\$ 2.2]$. The redshifts of the other sources are indicated by symbol type.

lation coefficient between $a$ and $b$ when $c$ and $d$ are held constant. We repeat this analysis for the same three assumed cosmologies, (i) $\Omega_{\mathrm{M}}=0$ and $\Omega_{\Lambda}=0$, (ii) $\Omega_{\mathrm{M}}=1$ and $\Omega_{\Lambda}=0$ and (iii) $\Omega_{\mathrm{M}}=0.1$ and $\Omega_{\Lambda}=0.9$. The dependences largely persist in all the cosmologies; we quantify and discuss the meaning of the dependence on cosmology of the strength of the anti-correlation of linear size with redshift in a forthcoming paper.

\subsection{A cautionary tale: using just one flux- limited sample}

To demonstrate how the tight correlation between luminosity and redshift in a single flux-limited sample can obfuscate the true dependences between these source properties and linear size and spectral index, we show in Table 5 the results of the statistical analysis carried out above on only the $3 \mathrm{C}$ sample (\$3.1). Although the relationship between linear size and spectral index is still discernible, (indicating that it is a real effect independent of luminosity or redshift) the extremely strong correlation between luminosity and redshift swamps the other dependences which do emerge when a number of complete samples with different flux-limits are taken together. 


\section{Parameterisation of the linear sizes}

Although we later demonstrate that there is no direct single physical mechanism by which linear size depends on redshift, for comparison of our data with work previously done by others we calculate the parametric dependence of the linear size on redshift and on luminosity with the form:

$$
D \propto(1+z)^{-n} P^{m} .
$$

Following Neeser et al. (1995), values of the exponent $n$ are found by multiplying individual source sizqs by $(1+z)^{n}$ until the conrelationcoefficient (hentce its associated significance) goes to zero. A formal error-bar is derived by considering the values of $n$ at which the significance of the correlation coefficient equals unity. The exponent $m$ was found in a similar manner. In Table 6 we list the various exponents thus derived. The notation we use is that $n\left[r_{a b \mid c d}\right]$ (or $\left.m\left[r_{a b \mid c d}\right]\right)$ is the exponent required to obtain a correlation coefficient of zero between quantities $a$ and $b$ when $c$ and $d$ are held constant. For comparison with previous studies, which have not included the spectral index dependence, we also present $n\left[r_{a b \mid c}\right]$ and $m\left[r_{a b l c}\right]$ which are the exponents required in equation 11 to make the 3 -way correlation coefficient between $a$ and $b$ equal zero, when $c$ is held constant.

\section{Comparison with previous work}

\subsection{The variation of linear size}

It has never been in dispute that the linear sizes of classical double radio sources are larger in the local Universe, although this has often come from studies restricted to very low redshifts. Indeed, this together with the fact that the angular sizes of classical double radio sources are relatively easy to measure led some to hope that it might be possible to use these objects as 'standard rods' to constrain cosmic geometry (see e.g. Ubachukwu (1995)), if it could be demonstrated that the linear size of an object is not influenced by its redshift. However, past reports of the true dependence of the linear sizes of radio sources have been equivocal as a brief survey of the literature indicates:

- Kapahi (1987) found a strong evolution in linear sizes of the form $D \propto(1+z)^{-3 \pm 0.5}$ for a cosmology with $\Omega_{\mathrm{M}}=1$ and $\Omega_{\Lambda}=0$, but at constant redshift he found that $D \propto P^{0.3 \pm 0.1}$. This was for a set of FR II radio galaxies from four samples: (i) The BDFL sample (Bridle et al. 1972) selected at $1.4 \mathrm{GHz}$; these galaxies had almost complete spectroscopic information and have $0.08<z<0.2$. (ii) The MC-I and MC-II samples (Machalski \& Condon 1983; Machalski \& Condon 1985) selected at $1.4 \mathrm{GHz}$. Out of these two samples only 4 galaxies had spectroscopic redshifts; redshift estimates from $R$ band magnitudes were used and redshift limits inferred for the two samples were 0.4 and 0.6 respectively. (iii) The LBDS sample which was selected at $1.4 \mathrm{GHz}$ by Windhorst, van Heerde, \& Katgert (1984). For the analysis Kapahi presented, all sources in this sample were deemed to have a redshift of 1.25. (iv) Galaxies from the revised 3CR sample which was selected at $178 \mathrm{MHz}$ by Laing et al. (1983).

- Barthel \& Miley (1988) found a weaker, and more explicitly uncertain, dependence of linear size on redshift and essentially no dependence on luminosity $\left(D \propto(1+z)^{-1.5 \pm 1.4} P^{-0.03 \pm 0.3}\right)$ from a sample of 134 steep spectrum radio quasars from Hewitt \& Burbidge (1980) known to exhibit radio emission. In the context of finding for their sample that distant quasars have a more bent and distorted appearance than nearby ones, they postulated that the ambient medium, interacting with the radio jets, played an important rôle in governing linear size evolution.

- Singal (1993) found for radio galaxies in his chosen sample that there was a strong anticorrelation of linear size with redshift but a correlation with luminosity $\left[D \propto(1+z)^{-3.0} P^{+0.35}\right]$, while for quasars an anti-correlation of linear size and luminosity was found as was marginal evidence for a dependence of linear size on redshift. His sample consisted of 789 radio sources, taken from 3CRR selected at $178 \mathrm{MHz}$ by Laing et al. (1983), the 1-Jy sample selected at 408 MHz (Allington-Smith et al. 1988; Lilly 1989), and the Molonglo (McCarthy et al. 1991) and B3 samples (Vigotti et al. 1989; McCarthy 1991) which were selected at $408 \mathrm{MHz}$.

- Oort, Katgert \& Windhorst (1987) found that the dependence of linear size on luminosity and on redshift, assuming that $\Omega_{\mathrm{M}}=0$, was described by: $D \propto(1+z)^{-3.3 \pm 0.5} P^{0.3 \pm 0.05}$. Their 
analysis was based on 163 radio galaxies from a number of different samples which included FR I type radio galaxies.

- Nilsson et al. (1993) found from a sample of 540 FR II double sources taken from the literature that for (high power) radio galaxies and quasars linear size and power are anti-correlated; they did not find any evidence for linear size evolution.

- The first study to use only complete samples, and using almost entirely spectroscopic redshifts, was that by Neeser et al. (1995). Using the revised $3 \mathrm{CR}$ and $6 \mathrm{C}$ samples [Laing et al. (1983); Eales (1985)], described in $\$ 3.1$ and $\$ 3.2$ respectively, they found $D \propto(1+z)^{-1.2 \pm 0.5}$ for $\Omega_{\mathrm{M}}=0$ and $\Omega_{\Lambda}=0$ and $D \propto(1+z)^{-1.7 \pm 0.5}$ for $\Omega_{\mathrm{M}}=1$ and $\Omega_{\Lambda}=0$. They found no intrinsic correlation between size and radio luminosity. Neeser et al. (1995) suggested the presence of dense line-emitting gas as being responsible for the observed linear size evolution. They also pointed out that such an effect could be achieved if radio jets were switched on for shorter periods of time at high redshift than at low redshift.

- A recent study (Buchalter et al. 1998) was based on the $1.4 \mathrm{GHz}$ FIRST survey; this study focussed on a sample of 103 quasars from the quasar catalogue of Hewitt \& Burbidge (1980) which had radio counterparts in the FIRST survey. In complete contrast with Neeser et al. (1995), they found no evidence for a dependence of linear size on redshift, other than that which arose from an anti-correlation of linear size with luminosity.

It is clear that sample inhomogeneity and redshift estimates rather than measurements, as examined in detail by Neeser et al. (1995), have played a significant rôle in giving rise to this plethora of contradictory results.

\subsection{Spectral index dependences}

Almost 40 years ago, evidence for a relationship between spectral index and luminosity was suggested by Heeschen (1960). Since that time other studies have confirmed his initial findings, e.g. Blumenthal \& Miley (1979) studied 3C and 4C sources, though they were unable to identify whether the dominant dependence was on redshift or on radio power.

Laing \& Peacock (1980) reported that for sources with hotspots, spectral index and the 'degree of spectral curvature' were correlated with luminosity. Their study was based on two complete samples of radio sources: one was 3CR (Bennett 1962) selected at 178 $\mathrm{MHz}$ and the other was selected at $2.7 \mathrm{GHz}$ [later published by Peacock and Wall (1985)]. They were similarly unable to say whether the dependence of spectral index was predominantly with luminosity or with redshift. Krolik \& Chen (1991) discussed a number of causes of these results: (i) since a typical radio galaxy spectrum steepens to higher frequency, in part a simple redshifting effect will give rise to the observed correlation between redshift and spectral index. They also pointed out (ii) the importance of inverse Compton losses in higher redshift sources which will give rise to steeper spectrum sources and (iii) the fact that an increased magnetic field which will increase the synchrotron emission (and hence increase the likelihood of a source being above a survey flux-limit) will also increase the rate at which spectral steepening occurs. No $K$-corrections were made to Laing \& Peacock's (1980) spectra so the correlation they found probably arose from some combination of all three of the causes discussed by Krolik \& Chen (1991). Employing (a large proportion of) redshift estimates for a faint (8C-selected) sample, Lacy et al. (1993) showed that high-frequency $(2 \mathrm{GHz})$ spectral index correlated more closely with redshift than with luminosity pointing towards the dominant importance of inverse Compton losses on high-frequency spectra.

\section{Modelling the complete samples}

In order to gain insight into the distribution of objects in the six planes $[P-D, P-z, P-\alpha, D-z, D-\alpha$ and $z-\alpha]$ of the $[P D z \alpha]$ parameter space occupied by the radio sources which are members of our complete samples, we performed Monte-Carlo simulations which we describe later in this section. We begin in $\$ 8.1$ with a précis of assumptions about the basic properties of radio sources which bear the test of observations and numerical simulations with particular reference in $\$ 8.1 .3$ to the distinct rôles of the hotspot and the head in a radio source. In $\$ 8.3 .2$ we discuss the constraints we have on the way in which radio sources expand with time giving us the formalism for their linear sizes. We describe in 8.4 the change of 
the luminosity (and the radio spectra) with time, of classical double radio sources and produce tracks for individual objects in $\$ 8.5$. We then explain in $\$ 8.6$ our formalism for obtaining the redshift distribution, and then how the model properly takes into account the

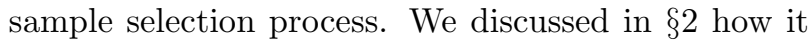
was imperative that a combination of complete samples should be used to infer the true dependences of radio source properties discussed in $\$$. A necessary condition for a satisfactory model of radio sources to explain the dependences from the combined samples is that it can reproduce the $[P D z \alpha]$ parameter space for each single flux-limited sample individually. We therefore conclude this section with a presentation of the six planes from the $[P D z \alpha]$ parameter space for each of a simulated and real dataset for the $3 \mathrm{C}$ and 7C complete samples.

\subsection{Assumptions about radio sources}

\subsubsection{Basic formalism for synchrotron radiation}

The synchrotron luminosity of an emitting region with volume $V$, threaded by magnetic field $B$ is given, in units of $\mathrm{W} \mathrm{Hz}^{-1} \mathrm{sr}^{-1}$, after averaging over pitch angles by:

$$
P_{\nu}=\frac{1}{6 \pi} \sigma_{\mathrm{e}} c \frac{B^{2}}{2 \mu_{\circ}} \frac{\gamma^{3}}{\nu} n(\gamma) V
$$

where $\gamma$ is the Lorentz factor of the electrons radiating at frequency $\nu, n(\gamma)$ is defined in terms of $n(\gamma) d \gamma$ as the number of electrons per unit volume per unit Lorentz factor between $\gamma$ and $\gamma+d \gamma, \sigma_{\mathrm{e}}$ is the Thomson scattering cross-section, $\mu_{\circ}$ is the permeability of free-space and $c$ is the speed of light. Adopting a delta-function approximation for the spectral emission from a given relativistic electron, a given frequency of emission $\nu$ is related to the Lorentz factor of the radiating electron by:

$$
\gamma=\left(\frac{m_{\mathrm{e}}}{e B} 2 \pi \nu\right)^{\frac{1}{2}}
$$

where $m_{\mathrm{e}}$ is the rest mass of an electron and $e$ is the charge on an electron. Although it is the case that a delta function profile is not a good representation of the power spectrum radiated by a synchrotronemitting particle (particularly in the high- $\gamma$ regime), as long as the underlying energy distribution of the radiating particles is smooth, then the energy emitted by the population at a particular frequency can be reasonably calculated using this approximation. The approximation will be particularly poor close to energy cutoffs.

\subsubsection{Jet behaviour}

We assume throughout a model in which a jet emanating from the central engine of an Active Galactic Nucleus (AGN), which transports a total power $Q_{\mathrm{o}}$, remains relativistic all the way from the central engine to the jet-shock (see Wardle \& Aaron 1997) and that the mechanism for confining the jet is via pressure balance with the lobe. We further assume that the jet has a density much lower than the external density [supporting evidence for this assumption comes from the simulations of Lind et al. (1989) and Clarke, Norman, \& Burns (1989)]. In the case of a low density jet, simple balancing of the momentum flux enabled Begelman, Blandford \& Rees (1984) to argue that the jet-shock advances at much less than the speed of light and consequently a large fraction of the bulk kinetic energy must be converted to thermal energy in the post-jet shock. We assume as indicated by the simulations of Lind et al. (1989) that the jet thrust is applied (even instantaneously) over an area significantly larger than the cross-sectional area of the jet itself and we identify this region with the hotspot, as discussed in the next section.

\subsubsection{Heads and hotspots}

We now carefully describe our usage of the term hotspot: we do not use the term hotspot merely to mean the bright emission in the outermost parts (the 'heads') of the lobes. We wish to refine the definition of hotspot from that which arises purely empirically (see e.g., Bridle et al. 1994). We use the term to refer to that region in which a distinct physical phenomenon occurs, namely emission associated with the post-jet shock structure (i.e. the extremely high magnetic field region within and just beyond the shock structure where a very large fraction of the bulk kinetic energy from the jet is thermalised and where particle acceleration may occur). A cartoon illustrating this is shown in Figure 9 .

Although many high-resolution high-fidelity images of hotspots reveal complex structure (Leahy et al. 1997, Hardcastle et al. 1997 and Black et al. 1992), we find no evidence in a comparison of the highluminosity quasars studied by Bridle et al (1994) with the lower-luminosity objects studied by Leahy et al. 


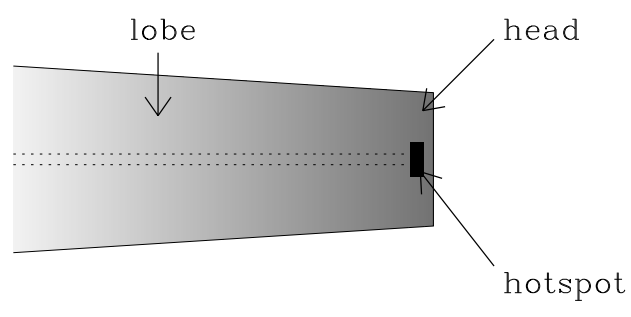

Fig. 9.- A schematic illustration of the basic features of a radio source which we wish to model: one half of a radio source is shown. Close to the outermost edge of the radio source is the hotspot where the bulk kinetic energy of the jet is thermalised and where particle acceleration may take place; the hotspot feeds the 'head' of the source, and it is the head pressure which governs the rate at which the source grows into its environment. The decreasing depth of the grey colour in the lobe represents the decrease in pressure between the head of the source back towards the active nucleus - the pressure gradient which drives backflow of plasma from the head. Note that depending on the variation of ambient density with radius, that the pressure may increase once more as the host galaxy of the AGN is approached.

(1997) of any very strong dependence of hotspot size on lobe pressure; the hotspots are of order a few kpc in diameter in all known FR II sources.

We also note that whatever the complexities of the physics by which hotspots are governed, hotspots do not appear to grow self-similarly with the radio source: i.e. the hotspot sizes do not scale strongly with source length. We note that what we perceive hotspot sizes to be will depend on the extent to which the emission from each hotspot is Doppler boosted.

\subsubsection{Hotspot observables}

The properties of hotspots which we are able to measure from high resolution maps made with MERLIN or the VLA include the flux densities and the break frequencies in hotspots at $\mathrm{GHz}$ frequencies, angular sizes subtended by hotspots (which in reality are probably lower limits because Doppler boosting of the plasma emerging through the hotspots will tend to make hotspots appear smaller). From these properties we are able to estimate the magnetic field strengths and pressures. (Estimates of hotspot magnetic fields may be made using the assumption of equipartition in the hotspots. X-ray detections of hotspots now give considerable confidence in the validity of the assumption of equipartition magnetic field strengths in hotspots (values which are in the region of tens of $\mathrm{nT}$ ) [see Harris, Leighly \& Leahy 1998].)

We describe in 88.1 .5 our justification for neglecting the direct contribution of the hotspots' luminosity to the overall luminosity measured from a radio source at a survey frequency like $151 \mathrm{MHz}$. Nonetheless the hotspot and its magnetic field play a vital role in governing the energy spectra of particles injected into the lobe, and hence the spectrum of the total radio luminosity we observe.

\subsubsection{The contribution of hotspots to the total lumi- nosity}

We contend that at low frequencies like $151 \mathrm{MHz}$, the luminosity from a hotspot never dominates over the luminosity from the head and the lobe. Naïve application of equation 2, using typical magnetic fields inferred for hotspots (e.g. as derived from equation 11), for an energy distribution which extends down to particles with Lorentz factors of $\sim 1$, for typical hotspot volumes ( $\sim$ a few $\mathrm{kpc}$ in diameter) predicts for the highest jet-power objects hotspot luminosities which are higher than the observed total luminosities of radio sources. However, for frequencies of a few $\mathrm{GHz}$, the predicted luminosities appear to be much more closely matched with the luminosities measured for resolved hotspots in observations. Leahy et al. (1989) found for a sample of 3C sources that the highest power sources had hotspots whose spectra became flatter between $151 \mathrm{MHz}$ and $1.5 \mathrm{GHz}$. (For the calculated magnetic field strengths the synchrotron self-absorption turnover frequencies for emitting volumes with sizes of a few $\mathrm{kpc}$ are in the region of tens of $\mathrm{MHz}$.) They interpreted this as the manifestation of a low-energy cutoff in the distribution of the radiating particles giving a deficit of hotspot emission at $151 \mathrm{MHz}$. In the context of an electron-proton jet where particle acceleration occurs at the hotspot such a cutoff is likely to occur when the Lorentz factor is of the order of the ratio of the proton mass to the electron mass, since for a particle to be efficiently accelerated, its gyro-radius must be large compared with the shock thickness (which is of the order of the gyro-radius of the thermal material, i.e. the protons, present). Although the precise nature of the low-energy cutoff depends on details such as the 
incoming jet velocity, it is likely that the value of the minimum Lorentz factor does not change greatly from hotspot to hotspot. However, the cutoff in frequency at which this is manifested does vary from hotspot to hotspot since for a given minimum Lorentz factor the minimum emitted frequency is proportional to the magnetic field in the hotspot (equation 3). For those sources whose hotspots have higher magnetic fields (which we will argue via equation 11 are those with higher jet-powers), the minimum frequency at which significant radiation will be observed will be higher than for sources with lower hotspot magnetic fields (and lower jet-powers). Such reasoning is consistent with the low contribution $(0.04 \%)$ the hotspots make to the total $327-\mathrm{MHz}$ luminosity in the archetypically high- $Q_{\text {o }}$ object, Cygnus A (Carilli et al. 1991).

\subsubsection{Inverse Compton losses arising from the AGN's own radiation field}

We now consider whether, for simulating low radio frequency surveys of the type described earlier in this paper, it is acceptable to neglect energy losses due to inverse Compton scattering off the AGN's own radiation field. This mechanism was invoked by Blundell \& Lacy (1995) to explain the steep spectrum in the $\mathrm{GHz}$ regime of the extended radio emission around the radio-quiet quasar E $1821+643$, and by Brunetti et al. (1997) to explain the soft X-ray emission detected in six 3C FRII radio galaxies. An 'equivalent magnetic field' may be derived from the energy density in the radiation field; this equivalent magnetic field falls off as $1 / r$ where $r$ is the distance out from the AGN. Significant losses arising from this mechanism will therefore be confined to some specific spatial extent. If this mechanism causes spectral steepening to be manifested at frequencies which may be redshifted to be close to the survey frequency, then its contribution cannot be neglected; we now investigate whether this could be the case.

The energy density of the AGN radiation field $U_{\text {rad }}$ is given by: $U_{\text {rad }}=Q_{\text {phot }} /\left(4 \pi c r^{2}\right)$ where $Q_{\text {phot }}$ is the photo-ionising luminosity. We assume that $Q_{\text {phot }} \sim \phi Q_{0}$ where $Q_{\mathrm{o}}$ is the total kinetic energy transported by one jet and $\phi$ is a factor which Willott et al. (1998b) suggest might be as high as 20. Equating the $U_{\text {rad }}$ with $B_{\text {eq }}^{2} / 2 \mu_{0}$ we find,

$$
B_{\text {eq }}(r)=\left[\frac{\phi Q_{\mathrm{o}}}{c} \mu_{0} \frac{1}{2 \pi r^{2}}\right]^{\frac{1}{2}} .
$$

We are now in a position to identify the timescale over which this magnetic field will impact on the flux which may be detectable by a survey, at a given distance from the AGN. We plot in Figure 10 a thick solid line indicating the trajectory of a point moving out from the centre of the AGN at which the break frequency would lower to $1 \mathrm{GHz}$ (this will not directly impact on a survey at a frequency as low as $151 \mathrm{MHz}$, until the source redshifts are greater than 5 ) if the plasma bathed in this radiation field had resided there since the radiation field was switched on. [This therefore over-estimates the rate at which the break in the spectrum will advance out from the centre of the AGN.] This trajectory is calculated using equations 12 and 4 at each position $r$. We also plot a thin solid line which is the trajectory of one head advancing away from the AGN (as described by halving the total linear size expressed in equation (6) while the dashed line is the trajectory of the midpoint of one arm of the radio source. It is seen that the spatial point where the break frequency becomes as low as $1 \mathrm{GHz}$ is only reached after the midpoint of the lobe has advanced beyond this. Comparison of the Inter-Planetary Scintillation measurements of the low-frequency $(81.5 \mathrm{MHz})$ measurements of $3 \mathrm{C}$ objects by Purvis et al. (1987) with the flux densities of these objects at $86 \mathrm{MHz}$ of Laing \& Peacock (1980) suggest that roughly half of the low-frequency emission comes from the head of the source. For the other half of the emission coming from the lobe, a decreasing proportion comes from plasma with increasing proximity to the core. Thus, because of the specificity of the spatial extent of the equivalent magnetic field arising from the quasar radiation field, those regions which suffer the most from this loss mechanism are those which barely make a significant contribution to the overall low-frequency flux. The importance of this effect cannot be so simply analysed for sources which are smaller in extent than e.g. 10 kpc. However, such sources are known to expand very quickly [Owsianik \& Conway (1998)] and the radiating plasma in them is continually replenished by freshly injected synchrotron emitting particles. Thus we conclude that the effects of inverse Compton losses off the AGN's own radiation field should not be significant in the selection of sources detected in surveys conducted in these frequency regimes, although this mechanism may be relevant in the question of the 'docked tails' of radio sources — which we discuss in $\$ 9.2$. 


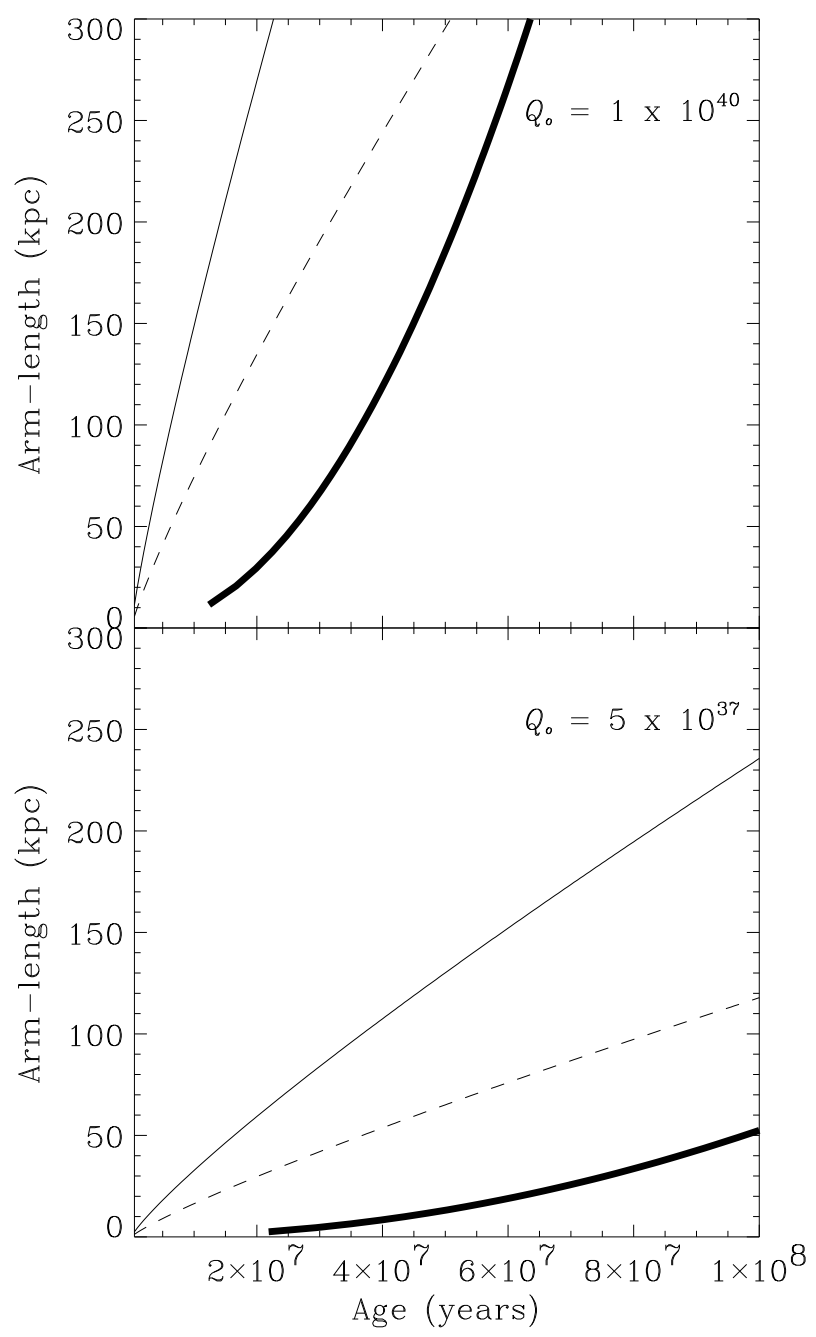

Fig. 10. - Thick solid line: the trajectory of a point moving out from the centre of the AGN at which the break frequency has lowered to $1 \mathrm{GHz}$; thin solid line: the trajectory of one hotspot advancing away from the AGN; dashed line: the trajectory of the mid-point of the arm of a source (see text for more details). Upper plot: trajectories for a source whose jets each carry total kinetic energy $Q_{\mathrm{o}}=10^{40} \mathrm{~W}$ and lower plot: with $Q_{\mathrm{o}}=5 \times 10^{37} \mathrm{~W}$; the value of $\phi$ was taken to be 5 .

\subsection{Environments}

An important probe of the densities of the environments of radio sources comes from studies of the depolarisation of the polarised intensity from their synchrotron emitting lobes. Such a study by Garrington \& Conway (1991) inferred the densities of particles responsible for the depolarisation to be consistent with the notion that radio galaxies are in poor group environments, with a typical value of the density given by $\rho_{\circ}=1.67 \times 10^{-23} \mathrm{~kg} \mathrm{~m}^{-3}$. We infer from Mulchaey \& Zabludoff (1998) for density profiles of such groups which are parameterised as follows:

$$
\rho(r)=\rho_{\circ}\left(\frac{r}{a_{\circ}}\right)^{-\beta}
$$

that suitable values of $\beta$ and $a_{\circ}$ are 1.5 and $10 \mathrm{kpc}$ respectively (see also Willott et al. 1998b).

Many authors have used various observational data as evidence for systematic changes in radio-source environments with redshift. Examples include: (i) the $\mathrm{X}$-ray detection of a number of high- $z 3 \mathrm{C}$ sources interpreted as evidence that a larger proportion of these lie in rich (X-ray emitting) clusters (Crawford \& Fabian 1996); (ii) a seeming increase in the optical richness of group/cluster environments with redshift (Hill \& Lilly 1991, Yates, Miller \& Peacock 1989); (iii) the identification of 3C host galaxies at $z \sim 1$ with the brightest cluster galaxies (Best et al. 1998, Eales et al. 1997, Roche et al. 1998) and (iv) an increase in the radio depolarisation of radio sources with either increasing redshift or luminosity (Garrington \& Conway 1991).

We have attempted, and failed, to synthesise these observations into a consensus picture for the evolution of radio-source environments. For example, the interpretation of X-ray detections associated with distant powerful radio sources as emission from hot cluster gas by Crawford \& Fabian (1996) is at odds with the interpretation of Brunetti et al. (1997) that inverse Compton scattering from either the AGN radiation field or from the cosmic microwave background is responsible for these X-rays. While the finding of Garrington \& Conway (1991) - that more distant sources show higher depolarisation - might appear to suggest that environments of more distant sources are more dense, it should be borne in mind that the more distant sources in their study, being shorter than lower- $z$ sources, inform on the depolarisation of lobe emission at spatial points much closer in the AGN, where inhomogenities due to the host galaxy and the possible recent merger products which led to the formation of the AGN will increase the measured depolarisation. In fact the interpretation of this very measurement is exacerbated by the "youth-redshift" degeneracy, a point to which we return in 9.5 .

In the absence of any clear consensus on this question we have preferred to adopt the simple assump- 
tion of no systematic change of radio-source environment with redshift and to see whether, when implemented in our model for radio sources, this necessarily presents contradictions with observed properties of radio-source samples. One might expect information on radio-source environments to improve rapidly with the advent of facilities such as AXAF.

\subsection{Source sizes}

\subsubsection{Source expansion}

The first essential step in modelling the time evolution of the properties of an FR II radio source is to determine how the size of the source depends on its age t. Falle (1991) and Komissarov \& Falle (1998) have shown that this dynamical problem can be solved via dimensional analysis, and since the mass involved is dominated by the ambient material swept-up within the bow-shock of the radio source the only characteristic quantities required are the kinetic power transported by one jet $Q_{\mathrm{o}}$, and $\rho_{0} \times a_{0}^{\beta}$. This gives:

$$
D(t)=2 c_{1} a_{\circ}\left(\frac{t^{3} Q_{\circ}}{a_{\circ}^{5} \rho_{\circ}}\right), \frac{1}{{ }^{5-\beta}}
$$

where $D(t)$ is the total length of a source, i.e. the hotspot to hotspot separation; see also Kaiser \& Alexander (1997). Assumptions about the radio source environment are therefore necessary (as summarised in $\$ 8.2$ ), but adopting these assumptions it is possible to constrain $c_{1}$ in three ways: (i) for those powerful FR II sources with measured expansion speeds $(v=0.5 d D / d t \approx 0.03 c$; Scheuer 1995, see $\$$ 8.3.2 an upper limit may be obtained, similarly from (ii) the distribution of radio sources in the $P-D$ plane of simulated samples (see Figures 17 and 19) and (iii) obtaining the axial ratios consistent with those observed. We take a value of 1.8 for the dimensionless constant $c_{1}$. Comparison of the observational and simulated data presented later in this paper (Figures $16 \& 17$ for the $3 \mathrm{C}$ data and Figures 18 \& 19 for the $7 \mathrm{C}$ data) will be used to show that $c_{1}$ must be similar to this value (see also Willott et al. 1998b). Falle's (1991) analysis also shows that the bow-shock must expand in a homologous (self-similar) manner.

We wish to re-emphasize the distinction between the hotspot and the head region of the expanding radio source (see $\$ 8.1 .3$ and Figure 9). The hotspot, also sometimes referred to as the 'working surface', may well move around the head of the lobe as in the "Dentist's Drill" model of Scheuer (1982) so that it is very dangerous to use the (instantaneous) properties of the compact hotspot to determine how the (much larger) head region of the source expands, e.g. by equating the pressure of the hotspot to the 'ram pressure' of the external medium [e.g. Readhead et al. (1996)]. It is better to consider the expansion of the whole head region, and its associated bow shock. Similar points have been made by Begelman \& Cioffi (1989).

From the jump conditions at a strong shock, (see e.g. Landau \& Lifshitz 1959) the pressure across the head $\left(p_{\text {head }}\right)$ is related to the pressure in the external medium $\left(p_{\text {ext }}\right)$ (whose adiabatic index is $\Gamma_{\mathrm{x}}=5 / 3$ ) in terms of the Mach number of the bow shock $\left(M_{\mathrm{b}}\right)$ by:

$$
\begin{aligned}
p_{\text {head }} & =p_{\text {ext }}\left(\frac{2 \Gamma_{\mathrm{x}} M_{\mathrm{b}}^{2}}{\Gamma_{\mathrm{x}}+1}-\frac{\Gamma_{\mathrm{x}}-1}{\Gamma_{\mathrm{x}}+1}\right) \\
& \approx p_{\text {ext }}\left(\frac{2 \Gamma_{\mathrm{x}} M_{\mathrm{b}}^{2}}{\Gamma_{\mathrm{x}}+1}\right) \quad \text { for } M_{\mathrm{b}} \gg 1
\end{aligned}
$$

In terms of the dimensional argument of Falle (1991) this may be expressed as:

$$
p_{\text {head }}=\frac{2}{\Gamma_{\mathrm{x}}+1} \rho_{\mathrm{ext}}\left(\frac{1}{2} \frac{d D}{d t}\right),^{2}
$$

where $D$ is given by equation 6 and $\rho_{\text {ext }}$ is the density of the external medium (identified with $\rho(r)$ and given by equation 5). This, together with equation 6, gives an expression for the pressure in the head at time $t$ :

$$
p_{\text {head }}=\frac{18}{\Gamma_{\mathrm{x}}+1} \frac{\rho_{\circ}}{(5-\beta)^{2}} c_{1}^{2-\beta} a_{\circ}^{2}\left(\frac{Q_{\circ}}{a_{\circ}^{5} \rho_{\circ}}\right)^{\frac{2-\beta}{5-\beta}} t^{\frac{-4-\beta}{5-\beta}} .
$$

Note that this is identical to equation 12 of Kaiser \& Alexander (1997), but that we are making a clear association between environmental ram pressure and the average value of internal pressure across the entire head of the source, and not just the compact hotspot. It is significant in this regard that high resolution radio imaging (Leahy et al. 1997, Hardcastle et al. 1997 and Black et al. 1992) suggests that, ignoring the possibly ephemeral hotspot emission, there is very little pressure variation across the head region of a given FR II radio source. It is also significant that the identification of the characteristic pressure with the whole head of a source, rather than the hotspot, avoids the 'lobe drag' problem posed, for example, by Williams (1991).

We believe that the material backflowing from the hotspot, via the head, inflates a cocoon which we 
identify with the observed radio lobe [an idea which dates back to Longair, Ryle and Scheuer (1973)] and which we assume confines the jet. Leahy \& Perley (1991) made images at $1.4 \mathrm{GHz}$ of 23 extragalactic radio sources with the VLA. In these images they detected the outer perimeter of the radio emission in most sources; at these outer edges the emissivity does not fade slowly into the noise, rather it suggests lobes which are over-pressured with respect to their local environment and will therefore expand outwards as the source ages, and indeed as it expands longitudinally.

Simulations show that jets with a low density ratio and high Mach numbers must produce backflow (Norman et al. 1982). Observational evidence for backflow, and high backflow speeds, comes from the spectral ageing analysis of Liu, Pooley \& Riley (1992) which found lobe-speeds (i.e. the anti-vector sum of the oppositely directed head advance speed and the backflow speed) in some sources to exceed $0.2 c$. When this is taken together with Scheuer's (1995) analysis of arm-length asymmetries arising from light-travel time effects which limit the head advance speed to substantially below this, perhaps as low as $0.03 c$ (see discussion of his method in $\$ 8.3 .2$ ), the lobe-speeds which Liu et al. derive strongly point towards high backflow speeds out of the hotspot. This, in turn, is strong evidence for a pressure gradient from the head of the source back towards the central nucleus.

If one considers a slice in low-frequency surface brightness through a radio lobe, along the jet axis, a gradual fall-off in surface brightness is seen from the head of the source back towards the core [see e.g. the slices shown in Leahy et al. (1989)]. Over a given volume, pressure is related to surface-brightness under equipartition conditions by a simple power-law scaling: $p \propto S^{4 / 7}$. Thus we may infer a gradual decline in pressure from the head towards the core and that the head pressure governs the lobe pressure.

The ratio of the pressure in the head to that in the lobe is not a well-defined quantity, but in the interests of simplicity in the modelling which we now describe, we will take for sources of all jet-powers a constant ratio of six for these pressures.

\subsubsection{Source advance}

A lower limit to the speed at which heads advance away from their AGN, and hence the rate at which radio sources grow, comes from the fact that FR II radio sources end in shocks which mean that the advance speed must exceed the ambient sound speed. The sound speed in an ionized gas at temperature $T$ $\mathrm{K}$ is given by $\approx 5 \times 10^{-7} \times c \sqrt{T}$ where $c$ is the speed of light; for $T=10^{7} \mathrm{~K}$ this gives an advance speed of $0.0015 c$.

Upper limits to the advance speed come from consideration of the asymmetries in arm-lengths of radio sources. This kind of analysis was first done by Longair \& Riley (1979); a subsequent and similar study was done by Best et al. (1995). These studies assumed that all arm-length asymmetries arose from geometrical projection and light-travel time effects. No account was taken of effects arising from environmental asymmetries. The former study obtained an upper limit to the head advance speed of $0.2 c$ while the latter study, which also took into account the effects of possible misalignment in the directions of ejection of the jets, obtained an upper limit of $\sim 0.4 c$. These values should very much be regarded as upper limits; tighter constraints were obtained by Scheuer (1995) who considered a sample of quasars each of which exhibited a considerable asymmetry in the presence of jets. The side of the brighter jet he ascribed as the nearer to us, attributing the jet brightness asymmetry to Doppler boosting. Arm-length asymmetries in these objects could then be more confidently attributed to light-travel time effects since there was a certain marker as to which was the nearer side to Earth. Scheuer's upper limit was somewhat sample dependent hinting at a non-constant hotspot advance speed, but gave a value of $0.03 \mathrm{c}$ for two samples and for all of his samples taken together a certain upper limit of $0.15 c$. This method of considering asymmetries, gives an 'instantaneous' measure of the speed at which the heads were advancing out from the active nucleus at the time when the light we observe was emitted from the radio source. The term 'instantaneous' is used somewhat liberally here, to mean 'within the light-travel time from the further hotspot to the nearer' as distinct from the speed which would be derived by averaging over the life-time of the source by dividing the (projected) length of the radio source

${ }^{5}$ The first direct measurements of source expansion were made by Owsianik \& Conway (1998) from multi-epoch global VLBI observations; they measured an advance speed of the CSO $0710+439$ to be $\gtrsim 0.24 c$. In such a small source (its linear size is $101.5 h^{-1} \mathrm{pc}$ ) it is likely that the expansion is strongly determined by the local density profile within the core radius of its host galaxy and so the environmental assumptions of \$8.2 would be correspondingly poor. 
by its total age.

Speed limits in the same ball-park as Scheuer's smaller limits were obtained by Readhead et al. (1996) who investigated the empirical relationships between the external density and the size, pressure, advance speed and flux densities of compact symmetric objects by assuming the sources to be ram pressure confined. They argued that hotspot pressures adjusted to the ambient density so that head advance speeds could be constant and close to $0.02 c$ (but see comments in $\S 8.3 .1$.

De Young (1997) has pointed out that the energy density at the end of the jet used to determine advance speeds could be the result of cumulative effects of the jet-environment interaction over the jet length. He suggests a preliminary approximation for how the advance speed depends on lossy jet propagation, namely by considering a constant entrainment rate per unit length of jet. He derives from this a roughly constant jet propagation rate.

We chose to adopt the formalism for the linear size growth developed by Falle (1991) on dimensional arguments discussed in $\$ .3 .1$.

The jet-axes of the sources we simulate in our models are deemed to be randomly oriented with respect to our line-of-sight. Each source is allocated an angle to the line of sight which is drawn from a distribution uniform in $(1-\cos \theta)$. The projected linear size of each simulated object is derived by evaluating $D$, the absolute physical size of the radio source, given in equation $\sqrt{6}$ at the time when the source intercepts our light-cone, and multiplying this length by $\sin \theta$.

In our model we deem that all radio sources will have their beams switched on for some maximum length of time $\left(t_{\text {max-age }}\right.$, which has been taken as $5 \times 10^{8}$ years). The time at which we 'make the observation' (i.e., examine whether the source is above the flux-limit of our simulated survey) of a given source is randomly chosen from a distribution described in $\S 8.6$, between 0 years and $t_{\max -\text { age }}$ years. If $t_{\max \text {-age }}$ were chosen to be too short, there would be two erroneous consequences: (i) if the true value of the coefficient of proportionality for the head advance $\left(c_{1}\right)$ is known then sources will never achieve projected linear sizes as large as those which are observed and (ii) a distribution of points would be generated on the $P-D$ plane, with an abrupt maximum size across the whole range in power, strikingly different from that which is observed. This would arise because the sampling of the radio sources would occur at early stages in their lives when very few sources would have fallen below the sample flux-limit. If the sampling of the radio sources broadly occurs over a time when the 'natural selection' process (the catastrophic decrease in luminosity due to synchrotron, adiabatic expansion and inverse Compton losses) governs the distribution of points on the $P-D$ plane, then it much more closely resembles that arising from real data.

If $t_{\max \text {-age }}$ is chosen to be too large, then provided the simulations are allowed to run through the birth of a sufficiently large number of radio sources there will be no consequence on the distribution of points on the $P-D$ plane. This is the case because the sampling of radio sources during the period of their lives when they are sufficiently bright to be detected by a survey on Earth is still random throughout all of this period. That there may be a relatively long period of time when radio sources have fallen below a sample fluxlimit during which our light-cone randomly samples them only has consequences for the number of sources which have to be 'observed' until the simulated survey is complete.

\subsection{Luminosity and spectral index develop- ment}

We took as our basic model for the luminositywith-time profile of a given radio source the model developed by Kaiser \& Alexander (1997) and Kaiser, Dennett-Thorpe \& Alexander (1997); this is the first model of extragalactic radio sources which directly incorporates the effects of inverse Compton losses on the luminosity of a source. This model assumes that source expansion is described by Falle's (1991) model, i.e. equation 6 .

\subsubsection{The rôle of hotspots in governing the lobe lu- minosity}

As described in $\$ 8.7$ each object in our model is assigned two jets which each transport a bulk kinetic power of $Q_{\mathrm{o}}$, assumed to be constant throughout the lifetime of the source.

Under the assumptions of $\$ 8.1 .2$ we can assume that the thrust of the jet is given by $\left(Q_{\circ} / c\right)$. The stagnation pressure $p_{\mathrm{s}}$ in the post-jet shock material will then be approximately given by:

$$
p_{\mathrm{s}}=\frac{Q_{\mathrm{o}}}{c A_{\mathrm{hs}}} \approx p_{\mathrm{hs}}
$$


where $A_{\mathrm{hs}}$ is the area perpendicular to the jet over which the jet thrust acts. We equate this stagnation pressure with $p_{\text {hs }}$, the pressure in the hotspot itself. If we assume that the energetics of the hotspot are described by equipartition, then the total energy density in the hotspot is due to two equal contributions, one from the particles and one from the magnetic field (this latter being equal to $B_{\mathrm{hs}}^{2} / 2 \mu_{\mathrm{o}}$ ). We may then express the magnetic field (assuming it to be tangled) in the hotspot $\left(B_{\mathrm{hs}}\right)$ in terms of the jet power as follows:

$$
\frac{Q_{\mathrm{o}}}{c}=\frac{B_{\mathrm{hs}}^{2}}{3 \mu_{\mathrm{o}}} A_{\mathrm{hs}},
$$

since for a relativistic fluid, and also for a tangled magnetic field, pressure is one-third of the total energy density. The break frequency in the hotspot $\nu_{\mathrm{bh}}$, is inferred from the usual expression for the break frequency in a synchrotron source:

$$
\nu_{\mathrm{bh}}=\frac{(9 / 4) c_{7} B_{\mathrm{hs}}}{\left(B_{\mathrm{hs}}^{2}+B_{\mathrm{CMB}}^{2}\right)^{2} t_{\mathrm{s}}^{2}}
$$

where following Leahy (1991), the coefficient of proportionality $c_{7}$ is $1.12 \times 10^{3} \mathrm{nT}^{3} \mathrm{Myr}^{2} \mathrm{GHz}$ thus the hotspot magnetic field $B_{\mathrm{hs}}$ and equivalent magnetic field due to the cosmic microwave background $B_{\mathrm{CMB}}$ are measured in $\mathrm{nT}, t_{\mathrm{s}}$ the 'synchrotron age' of the distribution is measured in Myr and the break frequency of the hotspot $\nu_{\mathrm{bh}}$ is measured in GHz. In the context of our model, we assume that the synchrotron age of the radiating particles depends on how long the particles are exposed to the magnetic field of the hotspot before they escape into the lobe. Some effect of this type seems inescapable in any model for a hotspot [e.g. Heavens \& Meisenheimer 1987 and Eales, Alexander \& Duncan 1989]. The magnetic field has the dependence on $Q_{\mathrm{o}}$ given by equation 11 giving a value for the break frequency which depends just on $Q_{\mathrm{o}}$, if we make the assumption that hotspot sizes are constant (we assume for all our sources that the radius and depth of each hotspot are $2.5 \mathrm{kpc}$ and $1 \mathrm{kpc}$ respectively).

\subsubsection{Lobe luminosity}

Our formalism essentially follows the model of Kaiser et al. (1997) but with two important differences: (i) instead of assuming a constant injection index [given by $p=-\partial \log n(\gamma) / \partial \log \gamma$ ] of $p=2.14$ we assume that the injection index is governed by the breaks in the energy distribution of the particles injected into the lobe from the hotspot. 5 These are ultimately determined by the maximum and minimum particle dwell-times in the hotspot together with the magnetic field strength in the hotspot, which as seen in equation 11 directly depends on $Q_{\mathrm{o}}$ itself. (ii) We also assume that adiabatic expansion losses out of the hotspot are governed by the hotspot pressure as derived in equation 10, not by the pressure in the head of the source which governs the increase in arm-length of the source.

Note that this process of adiabatic expansion of the particles out of the hotspot into the lobes does not move the energy distribution of the lobes out of equipartition, if the adiabatic indices of the particles and of the fields are both $4 / 3$ (as is the case for a highly relativistic fluid and for the virtual photons of a magnetic field).

A continuum of spectra may be regarded as injected to the lobe as the supply of particles in the hotspot is tapped for those populations of particles which have spent different dwell-times in the hotspot and thus have different break frequencies. Since the magnetic field in the hotspot is governed by the jet power $Q_{\mathrm{o}}$, this ultimately governs the spectrum of particles injected into the lobes [this is the origin of the $P-\alpha$ correlation in our model].

We derive a break in the energy spectrum of synchrotron particles freshly injected into the lobe by consideration of the corresponding break in their frequency spectrum of synchrotron emission. Since spectra are unchanged in shape, though translated to lower amplitudes and lower frequencies, by adiabatic expansion (Scheuer \& Williams 1968) - e.g. from the hotspot into the lobe - the Lorentz factors of the break frequencies of the particles injected into the lobes at some time $t_{\text {inj }}$ may be derived. Scheuer \& Williams showed that if the factor by which the linear scale of the emitting population has changed after undergoing adiabatic expansion is $f$, then the factor relating the break frequency after expansion to that before is $f^{-4}$. It is assumed that at the instant that an elemental population of particles is injected from the hotspot into the lobe, they undergo expansion which is approximately adiabatic. The break frequency of the synchrotron population as it is injected into the lobe is related to its break frequency in the

${ }^{6}$ We remind the reader of the distinction we draw between injection index and acceleration index as discussed in \$5.1. 
hotspots by a factor of $\left(p_{\text {hs }} / p_{\text {lobe }}\left[t_{\text {inj }}\right]\right)^{-4 /(3 \Gamma)}$, where $\left(p_{\text {hs }} / p_{\text {lobe }}\left[t_{\text {inj }}\right]\right)^{1 /(3 \Gamma)}$ is their linear scaling factor $(\Gamma$ is the usual adiabatic index of 4/3) and where $t_{\text {inj }}$ is the time at which a population of particles is injected into the lobe. This may be expressed in terms of the magnetic energy densities instead of the pressures i.e., the break frequency of the synchrotron spectrum of the particles freshly injected into the lobes is $\left(B_{\mathrm{hs}}^{2} /\left(2 \mu_{\circ} u_{B}\left[t_{\mathrm{inj}}\right]\right)\right)^{-4 /(3 \Gamma)} \times \nu_{\mathrm{bh}}$ where $\nu_{\mathrm{bh}}$ was the break frequency in the hotspots and $u_{B}\left[t_{\mathrm{inj}}\right]$ is the magnetic energy density in the lobes at this time $t_{\text {inj }}$ (which is directly proportional to the pressure in the lobe and head given in equation 9).

The energy of such a particle, freshly injected into the lobe, which is emitting at the break frequency $\nu_{\mathrm{bl}}$ is therefore given by $\gamma_{\mathrm{bl}} m_{\circ} c^{2}$ where $\gamma_{\mathrm{bl}}$ is given by:

$\gamma_{\mathrm{bl}}\left[t_{\mathrm{inj}}\right]=\left(\frac{\nu_{\mathrm{bl}}\left[t_{\mathrm{inj}}\right]}{\nu_{\mathrm{g}}}\right)^{\frac{1}{2}}=\left(\frac{\nu_{\mathrm{bh}}}{\nu_{\mathrm{g}}}\right)^{\frac{1}{2}}\left(\frac{B_{\mathrm{hs}}^{2}}{2 \mu_{\circ} u_{B}\left[t_{\mathrm{inj}}\right]}\right)^{-\frac{2}{3 \Gamma}}$

where $\nu_{\mathrm{g}}$ is the non-relativistic gyro-frequency, given by $e B\left[t_{\text {inj }}\right] /\left(2 \pi m_{\mathrm{e}}\right)$.

The exponent of the energy spectrum in the low $\gamma$ regime $\left(p_{1}\right)$ is taken to be 2 (corresponding to a frequency spectral index $\alpha$ of 0.5 ) assuming the particle acceleration mechanism in the hotspots to be via the first-order Fermi mechanism (Bell 1978). We note however that it is quite likely that the value of the acceleration index might be lower than 0.5 (Heavens \& Drury 1988) although we do not implement values lower than this in our model.

The injection spectrum is derived from the two break frequencies in the hotspot emission at $\nu_{\mathrm{bs}}$ and $\nu_{\mathrm{bf}}$. The former is the point in the spectrum where the population of particles which has lingered for the longest time in the hotspot changes from a spectral index of $\alpha=0.5$ to 1 (or $p=2$ to 3 , since $p=2 \alpha+1$ ). The latter is the point in the spectrum where the population of particles which escapes most quickly from the magnetic field of the hotspot changes from a spectral index of $\alpha=0.5$ to 1 (or $p=2$ to 3 ).

We deem the maximum $\gamma$ (i.e. $\gamma_{\mathrm{bs}}$ ) in the $p=2$ regime to be the Lorentz factor corresponding to the break frequency of the electrons which linger longest in the strong magnetic field of the hotspot before being injected into the lobes.

The exponent of the energy spectrum in the high $\gamma$ regime $\left(p_{\mathrm{h}}\right)$ is taken to be 3 in identifying a hotspot as an example of continuous injection (Carilli et al. 1991). We deem the minimum $\gamma$ (which is $\gamma_{\mathrm{bf}}$ ) in

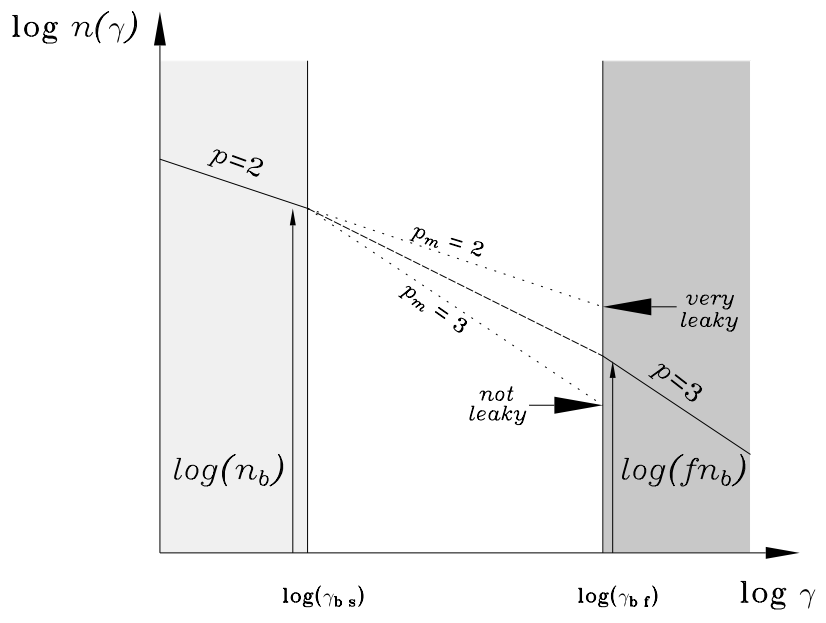

Fig. 11. - This schematic illustration shows the different energy regimes within a hotspot. The low energy spectrum is described by a region with index $p=2$ (in light grey). The high energy spectrum is governed by a region with index $p=3$ (in dark grey). The middle region is described by a single power law with index $p=p_{\mathrm{m}}$ (in white). The precise value of this index depends on how leaky the hotspot is deemed to be and the difference in dwell-times in the hotspot of the particles which leak out most quickly and those which leak out most slowly.

this regime to be the Lorentz factor corresponding to the break frequency of the electrons which leak out most quickly from the strong magnetic field of the hotspot into the lobe (see Figure 11). The shape of the spectrum between $\gamma_{\mathrm{bs}}$ and $\gamma_{\mathrm{bf}}$ is determined by the relative rates at which particles with different dwell-times in the hotspot leak out into the lobe.

A refined model of the details of this mechanism might suggest that this region should have quite a curved spectrum, but in the interests of simplicity, we approximate this region by a straight line whose gradient can take a maximum value of $3(\alpha=1)$ and a minimum value of $2(\alpha=0.5)$. 
The values of $\gamma_{\mathrm{bs}}$ and $\gamma_{\mathrm{bf}}$ are derived by considering the longest and shortest times for which particles reside under the influence of the magnetic field of the hotspot and deriving the appropriate break frequencies using equation 12. These dwell-times were taken to be $10^{5}$ years and 1 year respectively corresponding to, in the former case a backflow speed of $0.03 \mathrm{c}$ and, in the latter case, to particles which do not fully cross the hotspot. The break frequencies from these may be converted to Lorentz factors corresponding to breaks in the energy spectrum using equation 13. The derivation of $n_{\mathrm{b}}\left(=n\left[\gamma_{\mathrm{bs}}\right]\right.$ ) (the number density of particles with a given Lorentz factor $\gamma_{\mathrm{bs}}$ per unit Lorentz factor, see Figure 11) at a time $t_{\text {inj }}$ is obtained by equating the energy density at time $t_{\text {inj }}$ with the integral over the whole energy range, from $\gamma_{\min }$ to $\gamma_{\max }$ for each particle of energy $\gamma$ appropriately weighted as follows:

$$
u_{\mathrm{e}}\left[t_{\mathrm{inj}}\right]=\int_{\gamma_{\mathrm{bf}}}^{\gamma_{\max }} n_{\mathrm{h}}(\gamma) \gamma m_{\mathrm{e}} c^{2} d \gamma+\int_{\gamma_{\mathrm{bs}}}^{\gamma_{\mathrm{bf}}} n_{\mathrm{m}}(\gamma) \gamma m_{\mathrm{e}} c^{2} d \gamma+\int_{\gamma_{\min }}^{\gamma_{\mathrm{bs}}} n_{\mathrm{l}}(\gamma) \gamma m_{\mathrm{e}} c^{2} d \gamma
$$

with the weighting factors $n_{\mathrm{l}}(\gamma), n_{\mathrm{m}}(\gamma)$ and $n_{\mathrm{h}}(\gamma)$ from the three regimes in $\gamma$ respectively:

$$
\begin{array}{ll}
n_{\mathrm{l}}(\gamma)=n_{\mathrm{b}}\left(\frac{\gamma}{\gamma_{\mathrm{bs}}}\right)^{-2} \quad \text { if } \gamma \leq \gamma_{\mathrm{bs}}, \\
n_{\mathrm{m}}(\gamma)=n_{\mathrm{b}}\left(\frac{\gamma}{\gamma_{\mathrm{bs}}}\right)^{-p_{\mathrm{m}}} \quad \text { if } \gamma_{\mathrm{b} \mathrm{s}}<\gamma \leq \gamma_{\mathrm{bf}}, \\
n_{\mathrm{h}}(\gamma)=f n_{\mathrm{b}}\left(\frac{\gamma}{\gamma_{\mathrm{bf}}}\right)^{-3} \quad \text { if } \gamma>\gamma_{\mathrm{bf}}
\end{array}
$$

where $f=\left(\gamma_{\mathrm{bs}} / \gamma_{\mathrm{b} f}\right)^{p_{\mathrm{m}}}$, as shown in Figure 11 .

Thus we find that the normalisation factor $n_{\mathrm{b}}$ is given by:

$$
n_{\mathrm{b}}\left[t_{\mathrm{inj}}\right]=\frac{u_{\mathrm{e}}\left[t_{\mathrm{inj}}\right]}{m_{\mathrm{e}} c^{2}}\left(\gamma_{\mathrm{bs}}^{2}\left[\ln \frac{\gamma_{\mathrm{bs}}}{\gamma_{\min }}\right]+\frac{\gamma_{\mathrm{bs}}^{p_{\mathrm{m}}}}{p_{\mathrm{m}}-2}\left[\frac{1}{\gamma_{\mathrm{bs}}^{p_{\mathrm{m}}-2}}-\frac{1}{\gamma_{\mathrm{bf}}^{p_{\mathrm{m}}-2}}\right]+f \gamma_{\mathrm{bf}}^{3}\left[\frac{1}{\gamma_{\mathrm{bf}}}-\frac{1}{\gamma_{\max }}\right]\right)^{-1} .
$$

The energy density in the lobe at time $t, u_{\mathrm{e}}[t]$, is directly proportional to the pressure in the lobe and the head at time $t$, given in equation 9, with coefficients of proportionality which are of order unity (see Kaiser et al. 1997).

We take $\gamma_{\min }$ to be 1 and $\gamma_{\max }$, the maximum value of the Lorentz factor in the hotspots, to be $10^{14}$ since emission in the intense magnetic fields of the hotspots is observed to extend to frequencies of several tens of GHz.

We now consider the thermodynamics of the transition of the relativistic plasma from the jet, to the hotspot (meaning the post-jet shock region), and then through the head to its eventual home in the lobe itself. Following Kaiser et al. (1997) we equate the final energy state of an elemental volume $\delta V_{1}$ of plasma in the lobe with the energy it had when the jet delivered it to the hotspot (a dominant fraction of which is assumed to be in the form of bulk kinetic energy) minus the work done in adiabatically expanding from the hotspot to the lobe. We assume that the jet-power $Q_{\mathrm{o}}$ is completely converted into thermal energy at the hotspot. This seems reasonable given that the flow speeds into hotspots are close to $c$ (Wardle \& Aaron 1997) while those out of hotspots can be up to $\sim 0.2 c$ and are oppositely directed (Liu et al. 1992). [The energy lost to radiation mechanisms is neglected and can be shown to be many orders of magnitude smaller than the other quantities involved.] Hence,

$$
\frac{p_{1} \delta V_{1}}{\Gamma_{1}-1}=Q_{\mathrm{o}} \delta t_{\mathrm{inj}}+\left(\frac{p_{1} \delta V_{1}}{\Gamma_{1}-1}-\frac{p_{\mathrm{hs}} \delta V_{\mathrm{hs}}}{\Gamma_{\mathrm{hs}}-1}\right)
$$

where $\delta V_{\mathrm{l}}$ is the volume element injected into the lobe, $\delta V_{\mathrm{hs}}$ is the volume which this lobe element occupied when it was in the hotspot, $\delta t_{\text {inj }}$ is the time interval at $t_{\text {inj }}$ over which this element is injected into the lobe, $p_{\text {hs }}$ and $p_{1}$ are the pressure in the hotspot and lobe respectively and $\Gamma_{1}$ and $\Gamma_{\mathrm{hs}}$ are the adiabatic indices of the lobe and hotspot 
respectively each taken to be $4 / 3$. Since the expansion out of the hotspot is approximated to be adiabatic, we may write that:

$$
\frac{p_{\mathrm{hs}}}{p_{\mathrm{l}}} \frac{\delta V_{\mathrm{hs}}}{\delta V_{\mathrm{l}}}=\left(\frac{p_{\mathrm{hs}}}{p_{\mathrm{l}}}\right)^{\frac{\Gamma_{1}-1}{\Gamma_{\mathrm{l}}}}
$$

and using equation 10 we may thus write the contribution to the volume of the lobe from the element injected in $\delta t_{\text {inj }}$ at $t_{\text {inj }}$ as:

$$
\delta V_{\mathrm{l}}=\left[\frac{\left(\Gamma_{\mathrm{l}}-1\right) Q_{\mathrm{o}}}{p_{\mathrm{l}}}\left(\frac{Q_{\mathrm{o}}}{c A_{\mathrm{hs}} p_{\mathrm{l}}}\right)^{\frac{1-\Gamma_{\mathrm{l}}}{\Gamma_{\mathrm{l}}}}\right] \delta t_{\mathrm{inj}} .
$$

After multiplying by a term to allow for the further (adiabatic) expansion of the element as the source evolves (namely $\left[t / t_{\mathrm{inj}}\right]^{a_{1}}$, where $a_{1}$ is a constant which relates the expansion of volume $V$ after time $t$ as $V \propto t^{a_{1}}$ ), the integral of equation 19 over $t_{\text {inj }}$ may be identified as the volume $V_{l}(t)$ now occupied at time $t$ which has been injected into the lobe since time $t_{\min }$, and hence as the volume term in equation 2; this is given by:

$$
V_{\mathrm{l}}(t)=\int_{t_{\mathrm{min}}}^{t}\left[\frac{\left(\Gamma_{\mathrm{l}}-1\right) Q_{\mathrm{o}}}{p_{\mathrm{l}}\left[t_{\mathrm{inj}}\right]}\left(\frac{Q_{\mathrm{o}}}{c A_{\mathrm{hs}} p_{\mathrm{l}}\left[t_{\mathrm{inj}}\right]}\right)^{\frac{1-\Gamma_{1}}{\Gamma_{1}}}\right]\left(\frac{t}{t_{\mathrm{inj}}}\right)^{a_{1}} d t_{\mathrm{inj}}
$$

Note that the net result of this expansion is that only a fraction $\mu$ of the thermal energy deposited in the hotspots by the jet appears as stored energy in the lobes, the remainder being lost as work done against the shocked ambient medium in the bow-shock. The magnitude of $\mu$ can be estimated from equation 18 which is is essentially the reciprocal of the ratio of the internal energy of a given element in the lobe to that which it had in the hotspot. For $\Gamma_{\mathrm{l}}=\Gamma_{\mathrm{hs}}=4 / 3$, and typical values of $p_{\mathrm{hs}} / p_{\text {lobe }} \sim 10-100, \mu$ varies between $0.6-0.3$.

Figure 12 indicates the six possible ways in which the slope of the energy distribution of the relativistic particles injected into the lobe (i.e. the gradient at $\gamma_{\mathrm{inj}}$ ) may change with time, according to the relative value of $\gamma_{\mathrm{inj}}$ compared to $\gamma_{\mathrm{bs}}$ and $\gamma_{\mathrm{bf}}$.

We now consider the relation between the Lorentz factor of a particle injected into the lobe which will later contribute to the emission we observe at $t_{\mathrm{obs}}$, and its Lorentz factor at the time when the particle is injected. At $t_{\text {obs }}$ (the point when the light we observe leaves the object, i.e. when the object intercepts our light-cone) the Lorentz factor of the particles injected at that point in time, must be given by equation 3 for a given emitted frequency. The particles which contribute to the emission leaving the object at $t_{\mathrm{obs}}$ but which were injected into the lobe at a time $\Delta t$ prior to $t_{\mathrm{obs}}$ must have had a Lorentz factor higher when they were injected than they have at $t_{\mathrm{obs}}$, to compensate for the energy depletion during $\Delta t$. The maximum value of $\Delta t$ gives $t_{\mathrm{min}}$, the time before which no injected particles contribute to $t_{\mathrm{obs}}$. As in Kaiser et al. (1997) $t_{\mathrm{min}}$ is the time before which no particles which give rise to the emission observed (at the chosen frequency) at $t_{\mathrm{obs}}$ can be injected because the losses they suffer, given their Lorentz factors, would be too catastrophic. Thus for the ensemble of particles contributing to the radiation at a given emitted frequency at $t_{\mathrm{obs}}$, those with the largest Lorentz factors at injection are injected at $t_{\min }$ and those with the lowest are those actually injected at $t_{\mathrm{obs}}$.

We now consider the relation between the Lorentz factors injected at time $t_{\text {min }}$ and $t_{\text {obs }}$ and the corresponding breaks in the injected energy spectrum (depicted in Fig. 11). If the Lorentz factor of the injected electrons is always above these two or always below or always between them then the calculation is straightforward involving just one integral with the weighting function in each case being just either the bottom or the top or the middle form respectively of the full form of the weighting function listed in equation 15. If, during the time between $t_{\text {min }}$ and $t_{\text {obs }}$ the gamma-break should overlap with two distinct regimes in the spectrum, the integral must be performed in two steps. If during the time between $t_{\min }$ and $t_{\text {obs }}$ the gamma-break should overlap with all three distinct regimes in the spectrum, the integral must be performed in three steps (see Figure 12), thus: 

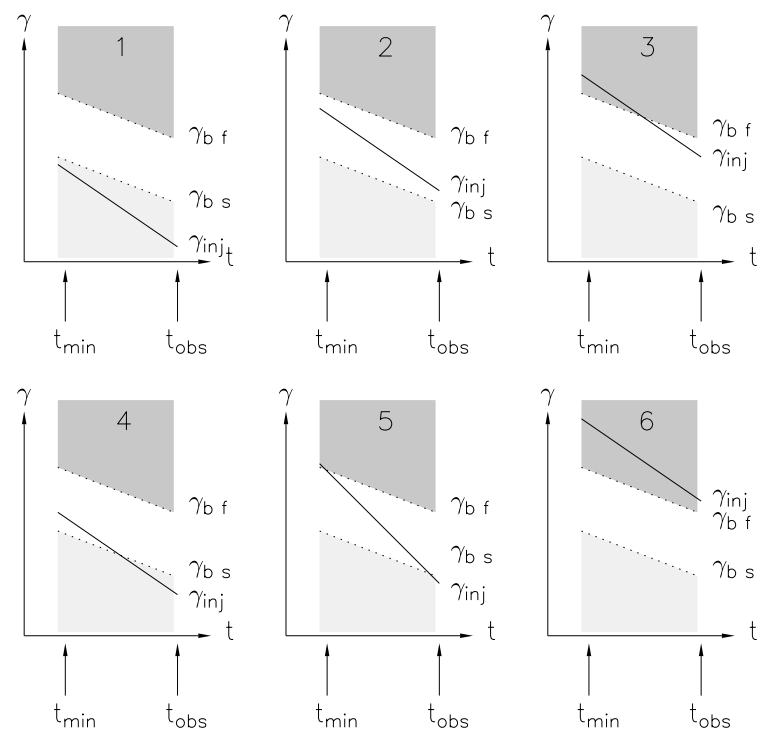

Fig. 12.- Schematic depiction of the six possible ways in which the Lorentz factor at injection of those particles which ultimately contribute to the radiation we observe (emitted at $t_{\mathrm{obs}}$ ) may relate to the Lorentz factors at the breaks in the injected spectrum. As in Figure 11, the light grey regions are where $p=2$, the dark grey regions are where $p=3$ and the white regions are where $p=p_{\mathrm{m}}$.

$$
\begin{aligned}
& P_{\nu}\left[t_{\mathrm{obs}}\right]=\frac{1}{6 \pi \nu} \sigma_{\mathrm{e}} c \times \\
& \left(\int_{t_{\gamma_{\mathrm{bs}}}}^{t_{\mathrm{obs}}} u_{\mathrm{B}}\left[t_{\mathrm{inj}}\right] \gamma^{3}\left[t_{\mathrm{inj}}\right] n_{\mathrm{b}}\left(\frac{\gamma\left[t_{\mathrm{inj}}\right]}{\gamma_{\mathrm{bs}}}\right)^{-2} \frac{Q_{\mathrm{o}}}{p_{\mathrm{l}}}\left(\frac{Q_{\mathrm{o}}}{c A_{\mathrm{hs}} p_{\mathrm{l}}}\right)^{\frac{1-\Gamma_{1}}{\Gamma_{1}}}\left(\frac{t_{\mathrm{obs}}}{t_{\mathrm{inj}}}\right)^{-a_{1}\left(1 / 3+\Gamma_{1}\right)} d t_{\mathrm{inj}}\right. \\
& +\quad \int_{t_{\gamma_{\mathrm{bf}}}}^{t_{\gamma_{\mathrm{bs}}}} u_{\mathrm{B}}\left[t_{\mathrm{inj}}\right] \gamma^{3}\left[t_{\mathrm{inj}}\right] n_{\mathrm{b}}\left(\frac{\gamma\left[t_{\mathrm{inj}}\right]}{\gamma_{\mathrm{bs}}}\right)^{-p_{\mathrm{m}}} \frac{Q_{\mathrm{o}}}{p_{\mathrm{l}}}\left(\frac{Q_{\mathrm{o}}}{c A_{\mathrm{hs}} p_{\mathrm{l}}}\right)^{\frac{1-\Gamma_{1}}{\Gamma_{\mathrm{l}}}}\left(\frac{t_{\mathrm{obs}}}{t_{\mathrm{inj}}}\right)^{-a_{1}\left(1 / 3+\Gamma_{\mathrm{l}}\right)} d t_{\mathrm{inj}} \\
& \left.+\quad \int_{t_{\text {min }}}^{t_{\gamma_{\mathrm{bf}}}} u_{\mathrm{B}}\left[t_{\mathrm{inj}}\right] \gamma^{3}\left[t_{\mathrm{inj}}\right] f n_{\mathrm{b}}\left(\frac{\gamma\left[t_{\mathrm{inj}}\right]}{\gamma_{\mathrm{bf}}}\right)^{-3} \frac{Q_{\mathrm{o}}}{p_{\mathrm{l}}}\left(\frac{Q_{\mathrm{o}}}{c A_{\mathrm{hs}} p_{\mathrm{l}}}\right)^{\frac{1-\Gamma_{\mathrm{l}}}{\Gamma_{\mathrm{l}}}}\left(\frac{t_{\mathrm{obs}}}{t_{\mathrm{inj}}}\right)^{-a_{1}\left(1 / 3+\Gamma_{\mathrm{l}}\right)} d t_{\mathrm{inj}}\right)
\end{aligned}
$$

where the volume element in equation 2 has been replaced by the integrand on the right-hand side of equation 20, and where $u_{\mathrm{B}}\left[t_{\mathrm{inj}}\right]$ represents the magnetic energy density in the lobe at the time a given element of particles is injected. The pressure in the lobe $p_{1}$ is taken to be a constant factor of six lower than the pressure in the head $p_{\text {head }}$ given by equation 8. We use Kaiser et al.'s (1997) expression for $\gamma\left[t_{\mathrm{inj}}\right]$ in their equation 10. 


\subsection{Predictions for the evolution of individ- ual sources}

\subsubsection{Sizes and energy budgets}

The volume of the radio source at the time of 'observation' may be found by integration of the volume elements which have been injected over the life of the radio source from a very early time in its life (e.g. $t_{\text {min }}=1 \times 10^{4}$ years $)$, up to $t_{\text {obs }}$, i.e. by performing the integral of equation 20, to allow for the expansion of these elements between the time they were injected and the time the 'observation' is made.

The energy transported, $E_{\mathrm{t}}$, by the jets in a radio source, after they have been switched on for a time $t$, is given simply by

$$
E_{\mathrm{t}}=2 Q_{\mathrm{o}} t
$$

The energy, $E_{\mathrm{s}}$, which is stored in the lobes according to the usual minimum energy arguments depends on the luminosity and the volume of the lobes as follows:

$$
E_{\mathrm{s}}=\chi P_{151}^{4 / 7} \frac{D^{9 / 7}}{R_{\mathrm{T}}^{6 / 7}}
$$

where $\chi$ is a constant throughout the life of a source which depends on assumptions about the low-energy cut-off of particles and their filling factor in the radio lobes (see Miley 1980), $P_{151}$ is the luminosity at rest-frame $151 \mathrm{MHz}, D$ is the total linear size of the radio source and where $R_{\mathrm{T}}$ is the axial ratio of the radio source, defined as the total length divided by the width, as one would measure on a radio map.

In our model, throughout a source's life, it does not store energy at a rate which is proportional to its age; this arises because the rate at which work is done by the expanding source itself depends on the age of the source. This follows from equation 18 and the fact that hotspot pressure does not scale with time in the same way as lobe pressure (unlike self-similar models). The prediction of axial ratios by our model comes from finding (the square root of $\pi / 4 \times$ ) the quotient of the cube of the linear size derived from equation 6 and the volume obtained from the integration of equation 20. We find that throughout the lifetime of an individual source its axial ratio steadily increases thus, for an individual source its expansion is not self-similar. For sources with $Q_{\mathrm{o}}=10^{38} \mathrm{~W}$, we find that the axial ratios increase from 2 to 8 , while for sources with $Q_{\mathrm{o}}=10^{40} \mathrm{~W}$, the axial ratios increase from 4 to 13 . Our model thus reproduces axial ratios which are larger in sources with higher jet-powers, consistent with the findings of Leahy \& Williams (1984) and Leahy et al. (1989) who found axial ratios between 4 and 12 (using the definition that the axial ratio is the total projected length of the source divided by its width). We note that the closer the jet-axis is to the line-of-sight, the smaller the measured axial ratio of a source will be because of projection effects. Both Leahy \& Williams and Leahy et al. found evidence of a trend of increasing axial ratio with increasing source luminosity and commented that the scatter was greater than that expected from projection effects alone. Leahy \& Williams' study was for a subset of the $3 \mathrm{C}$ sample (see $\$ 3.1$ ) with angular sizes greater than $45^{\prime \prime}$. With the elimination of these smaller (hence, given the sample, younger) sources it is then unsurprising in the context of our model that they found their more powerful sources to have higher axial ratios.

\subsection{2. $P-D$ tracks}

Figure 13 shows the tracks across the $P-D$ plane of sources according to our model described above with various values of jet-power and redshift and compares them with the predictions of the model of Kaiser et al. (1997). Note that in each case the tracks may be approximately described by two power-laws, either side of the knee. In each case on the left-hand side of the knee the gradient of our tracks are steeper than those of Kaiser et al. This arises because in our model the synchrotron emitting particles adiabatically expand out of a constant pressure hotspot into a lobe whose pressure falls throughout the source lifetime, thus the losses due to adiabatic expansion increase with time. In the model of Kaiser et al. (1997), the expansion of the synchrotron emitting population is from a head into a lobe whose pressures are in a constant ratio throughout the lifetime of the source. Thus the adiabatic losses in this latter model do not increase with time. The increasing adiabatic expansion losses with time in our model ensure that the tracks in the $P-D$ plane can be very steep even in an environment whose $\beta$ exponent is not particularly large (e.g. 1.5 here as described in 8.2 . This point plays a crucial rôle in determining the ease with which (particularly large, powerful) sources fall through the survey flux-limit.

The 'knee' between the two regimes of a $P-D$ track occurs earlier in the lives of sources at higher redshifts. Below the knee the magnetic field in the lobes $B_{\text {lobe }}$ is stronger than the equivalent field due to the 


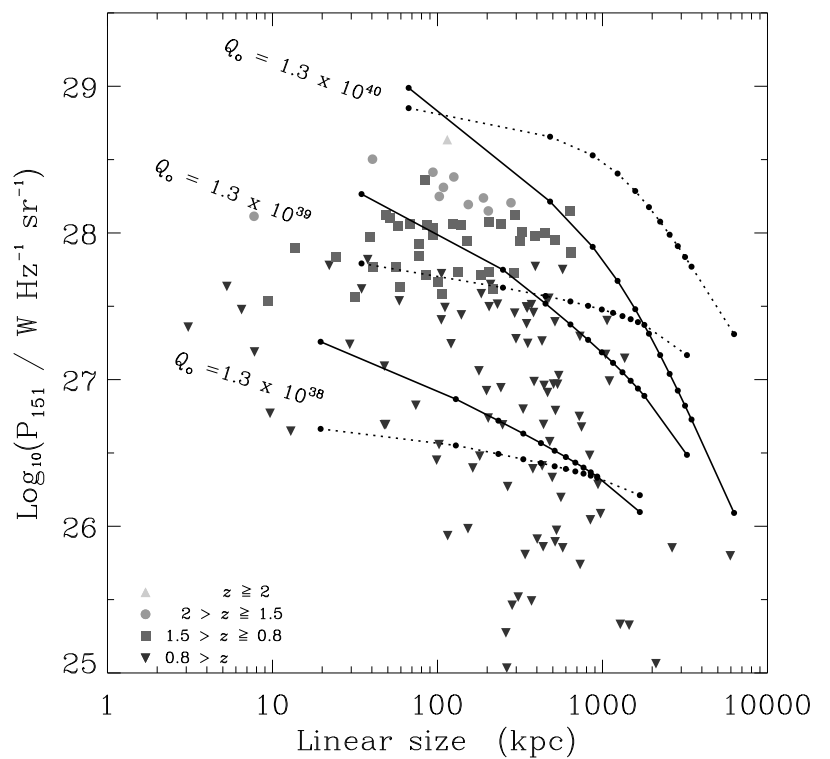

Fig. 13. - We overlay on this plot in solid lines the tracks through the $P-D$ plane of three sources (upper to lower) with $Q_{\mathrm{o}}=1.3 \times 10^{40} \mathrm{~W}$ at $z=2$, $Q_{\mathrm{o}}=1.3 \times 10^{39} \mathrm{~W}$ at $z=0.5, Q_{\mathrm{o}}=1.3 \times 10^{38} \mathrm{~W}$ at $z=0.2$. We overlay in dotted lines the tracks from the model of Kaiser et al. (1997) for sources with the same jet-powers and redshifts. The larger symbols in this plot show the rest-frame luminosities at $151 \mathrm{MHz}$ of members of the $3 \mathrm{C}$ complete sample (\$3.1) against their projected linear sizes. Since this is a logarithmic plot, the differences in the distributions between the projected and the true linear sizes of sources will be slight. Small dots lie on the tracks to indicate the times 1, 10, 20..100, 200 Myr through the source's life. All tracks in this figure were generated assuming an environment as described in $\$ 8.2$, i.e. with $\beta=1.5$ and with $c_{1}=1.8$.

cosmic microwave background $B_{\mathrm{CMB}}$. Spectral losses for a particle of Lorentz factor $\gamma$ go as $\gamma^{2} B_{\text {lobe }}^{2}$, but for a given $\gamma$ this rate of energy loss decreases with time since $B_{\text {lobe }}$ is decreasing with time. Throughout the lifetime of the source, $B_{\text {lobe }}$ will be falling thus for radiation at a fixed frequency which we might observe in our survey, the Lorentz factor $\gamma$ will be proportional to the reciprocal of the root of the lobe magnetic field (see equation 3). Thus as the source ages, increasingly energetic particles are responsible for the emission observed at some fixed frequency. Once the magnetic field of the lobe has dropped below $B_{\mathrm{CMB}}$, the inverse Compton losses will dominate the rate at which the high $\gamma$ particles are depleted, in this sec- ond regime in the source's life. This magnetic field is constant and not decreasing as $B_{\text {lobe }}$ is, so the spectral losses thereafter remain at a fixed, not a decreasing, rate. However, the lobe magnetic field $B_{\text {lobe }}$ continues to fall requiring yet higher Lorentz factors for emission at a given frequency, and the high Lorentz factor population is being depleted at a steady rate due to inverse Compton scattering from the cosmic microwave background.

Figure 14 shows tracks across the $P-D$ plane derived from our model for various values of jet-power and redshift. The solid lines of the tracks become dotted when the luminosity and redshift of that source are such that they fall below the survey flux-density limit of $0.5 \mathrm{Jy}$. Comparison of the time markers along the different tracks show that the tracks of the more powerful, higher-redshift sources become steeper, this together with the consequences of the flux-limit, means that these sources fall below a survey flux-limit at an earlier stage in their lives than do sources at lower power and redshift. Here then, is an indication of the origins of the effect we discuss in more detail in $\$ 9.5$, the "youth-redshift degeneracy" which is responsible for linear size evolution, on the assumption that radio sources are intercepted by our light-cone in a manner which is essentially random. We examine in detail the correct way to calculate the interception of the source population with our lightcone in $\$ 8.6$ and then see clearly how these two effects arise.

\subsection{Redshift distribution}

In this section we consider how to model the number of radio sources born at a sequence of cosmic times, and their subsequent interception with our light-cone. Successive populations of radio sources are hatched every $10^{6}$ years, and are evolved, starting at some initial high redshift ( $z=10$ is used).

We assume that the number of sources born at a given cosmic time $(t)$, per unit cosmic time, per unit co-moving volume element (defined as having a proper volume of one cubic Gpc at redshift zero) is given by $\rho(t) d t=\rho(z) d z$ which may be parameterised by:

$$
\rho(z) d z \propto \exp \frac{1}{2}\left(\frac{z-z_{1}}{\Delta z_{1}}\right)^{2} d z .
$$

We further assume that at any given cosmic time, this description holds at any spatial point in the Universe, since this is presumed to be homogeneous 


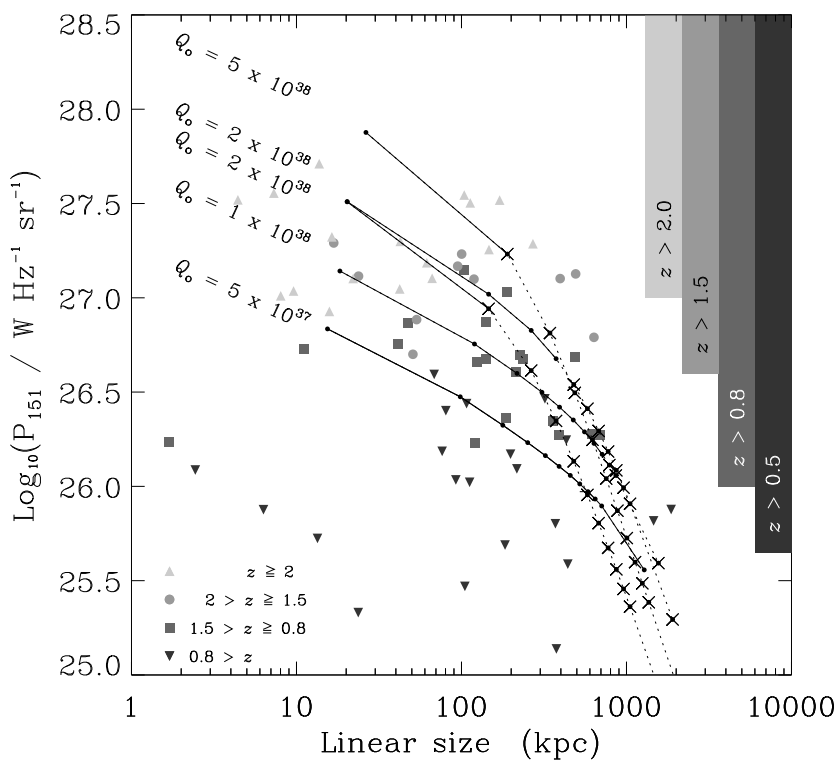

Fig. 14. - We overlay on this plot the tracks through the $P-D$ plane of five sources (upper to lower) with $Q_{\mathrm{o}}=5 \times 10^{38} \mathrm{~W}$ at $z=3, Q_{\mathrm{o}}=2 \times 10^{38} \mathrm{~W}$ at $z=1.5, Q_{\mathrm{o}}=2 \times 10^{38} \mathrm{~W}$ at $z=2.5, Q_{\mathrm{o}}=1 \times 10^{38}$ $\mathrm{W}$ at $z=0.8$, and $Q_{\mathrm{o}}=5 \times 10^{37} \mathrm{~W}$ at $z=0.5$. The left-most light-grey bar indicates that for an object to be brighter than the flux-limit of the $7 \mathrm{C}$ sample $(0.5 \mathrm{Jy})$, if it is at $z>2$ then its luminosity must be in the range indicated by the bar; the corresponding bars going to lower luminosities are for the lower redshifts $1.5,0.8$ and 0.5 respectively. The solid lines of the tracks become dotted when the luminosity and redshift of that source are such that they fall below the survey flux-limit of $0.5 \mathrm{Jy}$. The larger symbols in this plot show the rest-frame luminosities at $151 \mathrm{MHz}$ of members of the $7 \mathrm{C}$ complete sample (\$3.3) against their projected linear sizes. Small dots lie on the tracks to indicate the times 1, 10, 20...100, $200 \mathrm{Myr}$ through the source's life; these become cross-symbols when an object falls below the flux-limit. It can be seen that because the tracks of the more powerful, higher-redshift sources become steeper, and because of the consequences of the flux-limit, these sources fall below a survey flux-limit at an earlier stage in their lives than do sources at lower power and redshift. The same environment and value of $c_{1}$ are assumed as for Figure 13.

and isotropic. We make the approximation that all sources are precisely following the Hubble flow.

We wish to consider the total number of sources born at a given cosmic time which will be intercepted by our light cone, within a certain interval in cosmic time from the time when they were born. [This interval is chosen to be the maximum length of time for which the beams in each radio source are switched on. We assume that in each radio source this is $5 \times 10^{8}$ years (see justification of this number in $\$ .3 .2$ ), presuming no dependence on redshift or on intrinsic jetpower.] If a source born at a given point in cosmic time is to be intercepted by our light-cone at some point between the time when it was born and the time when its beam is switched off this has implications for the distance it must be from us at the point when it is born. If the source is intercepted by our light-cone at the time when it is born, then its distance from us is simply given by the distance along our light-cone to this redshift (which is hereafter referred to as $z_{\text {birth }}$ ). If the source is intercepted by our light-cone at the moment when the beam is switched off (i.e. $5 \times 10^{8}$ years after the source was born) then its distance from us at this later point in cosmic time must be the distance along the light-cone to this lower redshift, but this must correspond to a specific distance from us at the earlier cosmic time when the source was born. With this information a thick radial shell of a specific proper volume whose maximum and minimum radii are defined by the maximum distance and the minimum distance a source may be from us if it is born at a given $z_{\text {birth }}$ and if it is intercepted by our lightcone within a cosmic time of $5 \times 10^{8}$ years from $z_{\text {birth }}$. This region, for a given $z_{\text {birth }}$, is hereafter called the 'relevant co-moving volume element'. The relevant co-moving volume element is the maximum size, for a given solid angle, of a box which contains all the sources which must be intercepted within an interval of $5 \times 10^{8}$ years of being born at the redshift $z_{\text {birth }}$ (and no sources born at this redshift which are not intercepted by our light-cone within this interval).

The form of the metric which we use in this analysis is:

$d s^{2}=c^{2} d t^{2}-\frac{R^{2}(t)}{\left(1+\frac{1}{4} k r^{2}\right)^{2}}\left[d r^{2}+r^{2}\left(d \theta^{2}+\sin ^{2} \theta d \phi^{2}\right)\right]$,

where $R(t)$ is the scale factor of the Universe at cosmic time $t, r$ is a radial co-moving co-ordinate, $\theta$ and $\phi$ are the polar and azimuthal co-ordinates; $k$ refers to the curvature of the Universe $(k=0$ for a spatially flat Universe, $k=1$ for an open Universe and $k=-1$ for a closed Universe). Following standard practice, we introduce an alternative radial co-moving co-ordinate 
$\chi$ defined in terms of $r$ by:

$$
\begin{array}{lll}
\chi & =r & \text { if } k=0 \\
\sin \chi & =r /\left[1+r^{2} / 4\right] & \text { if } k=1 \\
\sinh \chi & =r /\left[1-r^{2} / 4\right] & \text { if } k=-1
\end{array}
$$

writing the space-time metric as

$$
d s^{2}=c^{2} d t^{2}-d l^{2},
$$

we have for $k=0$ :

$$
d l^{2}=R^{2}(t)\left[d \chi^{2}+\chi^{2}\left(d \theta^{2}+\sin ^{2} \theta d \phi^{2}\right)\right]
$$

while for $k=1$, we write:

$$
d l^{2}=R^{2}(t)\left[d \chi^{2}+\sin \chi^{2}\left(d \theta^{2}+\sin ^{2} \theta d \phi^{2}\right)\right],
$$

and for $k=-1$, we write:

$$
d l^{2}=R^{2}(t)\left[d \chi^{2}+\sinh \chi^{2}\left(d \theta^{2}+\sin ^{2} \theta d \phi^{2}\right)\right] .
$$

In the three cases the proper volume $\Delta V$ of a spherical shell, whose inner and outer edges are at $\chi_{1}$ and $\chi_{2}$ respectively, (a co-moving volume element) is given by:

$$
\Delta V= \begin{cases}2 \pi R^{3}(t)\left[\frac{2}{3} \chi^{3}\right]_{\chi_{1}}^{\chi_{2}} & \text { for } k=0 \\ 2 \pi R^{3}(t)\left[\chi-\frac{1}{2} \sin 2 \chi\right]_{\chi_{1}}^{\chi_{2}} & \text { for } k=+1 \\ 2 \pi R^{3}(t)\left[\frac{1}{2} \sinh 2 \chi-\chi\right]_{\chi_{1}}^{\chi_{2}} & \text { for } k=-1\end{cases}
$$

Along a light-ray, the interval $d s=0$, and along a radial light-ray

$$
d s^{2}=c^{2} d t^{2}-R^{2}(t) d \chi^{2}
$$

is independent of the value of $k$ so we may write

$$
\chi=c \int_{t}^{t_{\text {now }}} \frac{1}{R(t)} d t .
$$

The limits of integration, $\chi_{2}$ and $\chi_{1}$, are respectively the radial co-ordinates to the outer and inner surfaces of the thick radial shell and are given by:

$$
\chi_{2}=c \int_{t_{\mathrm{birth}}}^{t_{\mathrm{now}}} \frac{1}{R(t)} d t,
$$

and

$$
\chi_{1}=c \int_{t_{\mathrm{birth}}+5 \times 10^{8} \mathrm{yr}}^{t_{\mathrm{now}}} \frac{1}{R(t)} d t
$$

where $t_{\text {birth }}$ is the cosmic time when this batch of sources is born.

In order to convert proper volumes measured in cubic Gpc at the time of birth into units which will be cubic Gpc at redshift zero, the proper volumes are multiplied by $\left(1+z_{\text {birth }}\right)^{3}$.

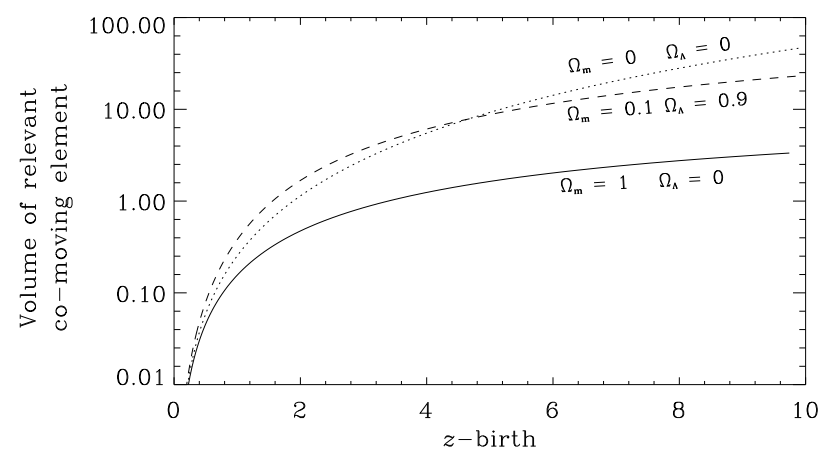

Fig. 15. - This figure shows the volume of the 'relevant co-moving element' (see text for definition; the units are those which will be cubic Gpc at redshift zero) at a given redshift, in each of the three different cosmological models we consider in this paper.

Once the volume of this co-moving element is calculated in a chosen cosmological model, it is then simple to calculate the number of sources born [per solid angle born in the chosen interval in cosmic time] at a given $z_{\text {birth }}$ which will ultimately be intercepted by our light-cone, by multiplying this by $\rho(z)$ given in equation 24. Precisely when each source intercepts our light-cone is determined by its radial distance from us at the cosmic time corresponding to $z_{\text {birth }}$ within the radial shell described above. Since we assume spatial homogeneity we deem these sources to be randomly distributed within the volume of the box. Thus the length of time which elapses after a given source is born before it intercepts the lightcone, (called $t_{\text {age }}$ ) is randomly drawn between 0 and $5 \times 10^{8}$ years, but not from a uniform distribution. The uniformity of space requires that within equal (infinitesimal) volumes within the thick radial shell there should be equal numbers of objects born at $z_{\text {birth }}$. Sources are randomly allocated to equal volume shells, then their radial co-ordinate $(\chi)$ is derived by inverting the equation 31 appropriate for the value of $k$ chosen, and solving this using an iterative method. We see that this process means that a source is therefore more likely to have a larger radial co- 
ordinate within a given spherical shell than a smaller one, since the sources are uniformly distributed in volume rather than in their radial co-ordinate, and hence there is a mild bias arising from geometry for a source to be seen at an earlier rather than a later stage in its life. Once the appropriate radial co-ordinate is drawn, the cosmic time $\left(t_{\text {obs }}\right)$ at which the source is intercepted by our light-cone may be determined from

$$
\chi=c \int_{\mathrm{t}_{\mathrm{obs}}}^{t_{\mathrm{now}}} \frac{1}{R(t)} d t
$$

By subtracting the cosmic time when the source is intercepted by the light-cone from the cosmic time when the source was born, the time for which a source should evolve i.e. $t_{\text {age }}$, according to its initial parameters, is known. Thus,

$$
t_{\text {age }}=t_{\text {obs }}-t_{\text {birth }} .
$$

Therefore, at a given point in cosmic time, we know the exact number of sources born which will intercept the light-cone thereafter within a maximum time-period of $5 \times 10^{8}$ years, and the length of time for which each evolves until the light ultimately observed by us is emitted. The redshift of such an object is then derived by considering the cosmic time at which the source was born, plus this time for which the source lived $\left(t_{\text {age }}\right)$ until the light which we ultimately observe on the Earth, left the source.

\subsection{Parameters and results}

A jet-power is allocated to each jet in a simulated object which has been drawn randomly from a distribution of the form:

$p\left(Q_{\mathrm{o}}\right) d Q_{\mathrm{o}}= \begin{cases}Q_{\mathrm{o}}^{-x} d Q_{\mathrm{o}} & \text { if } Q_{\min }<Q_{\mathrm{o}}<Q_{\max }, \\ 0 & \text { if } Q_{\mathrm{o}} \geq Q_{\max } \text { or } Q_{\mathrm{o}} \leq Q_{\min }\end{cases}$

where $x$ is a positive number. Each source is then allowed to evolve in luminosity (according to the prescription discussed in $\$ 8.4$ ) at a (rest-frame) frequency $(1+z) \times$ the observing frequency for the length of time $t_{\text {age }}$. If the luminosity of the source at this frequency is such that its flux is above the deemed survey fluxlimit (e.g., $0.5 \mathrm{Jy}$ for a simulated $7 \mathrm{C}$ sample) given its redshift, it is regarded as being detected by the survey. Sources which do not meet the survey flux-limit are thereafter ignored. Note that we do not simulate the effect of surface brightness, as distinct from flux density, in these simulated surveys. However, as Riley (1989) discusses the numbers of sources omitted from real surveys due to surface brightness effects in large angular size sources is small indeed.

For those sources which do lie within the sample flux-limits, the following calculations are performed: the luminosities at two other frequencies (including rest-frame $151 \mathrm{MHz}$ ), for the same point in the source's lifetime, are derived as is the projected linear size (according to the prescription discussed in 8.3.2). From three (luminosity, frequency) points now derived for each source in the sample, a quadratic expression may be fitted from which the spectral index at rest-frame $151 \mathrm{MHz}$ is derived, together with the $a_{1}$ and $a_{2}$ coefficients of spectral shape discussed in Section 4.2.

Figures 16 and 17 show real and simulated $3 \mathrm{C}$ data. Figures 18 and 19 show real and simulated $7 \mathrm{C}$ data. In these simulations the 'sky-areas' (i.e. the solid angle covered by each survey) differ by a factor of 430 , which is of the same order as the factor by which the true sky areas covered by $3 \mathrm{C}$ and $7 \mathrm{C}$ differ $(4.2 \mathrm{sr}$ versus $0.013 \mathrm{sr}$ ). For the simulations presented here the values of the $Q_{0}-$ related quantities included in equation 38 are $x=2.6, Q_{\max }=5 \times 10^{42} \mathrm{~W}$ and $Q_{\min }=5 \times 10^{37} \mathrm{~W}$. The values of the $z$-related quantities are $z_{1}=2.2$ and $\Delta z_{1}=0.6$.

\subsection{Discussion of simulations}

A comparison of the simulated and real $3 \mathrm{C}$ data suggests that to first order there is agreement in the basic quantities. Sources are detected out to approximately the same redshift, the maximum size of sources detected is approximately correct, the dependence between linear size and redshift shows encouraging correspondence and spectral indices increase with luminosity rather than with redshift. These correspondences also seem to be broadly present in the $7 \mathrm{C}$ datasets though there is of course considerably less scatter seen in the spectral index distributions; note that we have not included in our model the possibility that acceleration indices within the hotspot might be less than 0.5 [as suggested by the work of Heavens $\&$ Drury (1988)] so the small angular size, $\alpha<0.5$ objects from $3 \mathrm{C}$ with $\log _{10} P_{151} \sim 27.5$ will not appear in our simulations. Particularly in the case of the $7 \mathrm{C}$ sample, there is a discrepancy between the minimum spectral indices observed and those simulated and it is unlikely that all of the discrepant sources could be explained by invoking synchrotron self-absorbtion at these frequencies. In the case of the $3 \mathrm{C}$ sample, the simulation shown in Figure 17 has 
too high a proportion of sources with linear sizes $<10$ kpc. It is likely that the simplicity of our assumptions about the environments of radio sources at these very small separations from the AGN are responsible for this, in particular the rapidity of source expansion in these regimes may be underestimated (as discussed in \$8.3.2). We do not include in our simulations the analogues of those sources which we believe to be free-free absorbed or synchrotron self-absorbed which may be responsible for the absence of these small sources in real samples. The bimodality in the simulated spectral indices is likely to arise in part because we do not model any variation in hotspot size in the lifetime of an individual source, or in different sources.

We defer detailed statistical comparison of the differently parameterised simulated datasets and an investigation of the constraints on the birth-function in the different cosmological models and the purported existence of a redshift cutoff in the radio source population, to a forthcoming paper.

\section{Remarks on interpretation}

\subsection{Linear size evolution}

The anti-correlation of linear size with redshift arises naturally as a consequence of imposing a survey flux-limit on a population of sources each of whose luminosity falls off with age. For high-power sources at high-redshift, the $P-D$ tracks are seen to have steepening slopes throughout the life of the source. This arises partly because of the effect of inverse Compton losses to the cosmic microwave background (as found by Kaiser et al. 1997) and partly because of increasing adiabatic expansion losses of successively injected particles emerging from a constant pressure hotspot into a lobe whose pressure decreases throughout the life of the source. In the case of the highest power sources, the injection index itself is steeper which causes further steepening of the luminosity of a radio source with time. The interception of our light cone with a particular object is random throughout its lifetime, but high redshift sources in the later stages of their lives are increasingly less likely to be detected by surveys because of the steep decline of their luminosity with age. Thus the only high redshift objects we tend to detect are younger (more luminous) ones — which being younger, are shorter; hence 'linear size evolution' is seen. This means that in a survey, there is a strong correlation between youth and redshift in the sense that the higher the redshift of a source, the earlier in its lifetime we are observing it. Such a degeneracy must be taken into account when explaining "redshift effects", which we explore in $\S 9.5$.

\subsection{Injection indices and the $P-\alpha$ correlation}

The $P-\alpha$ correlation may arise in the way we have suggested with the injection spectrum of particles, and the steepness of this distribution, governed in a way which depends on the jet-power. It is possible however, that the acceleration index of immediate post-shock particles is not necessarily 0.5 as implied by first-order Fermi mechanisms, but that this spectrum itself is governed by the jet-power in some way depending on the detailed physical mechanisms of the hotspot. Whatever the details of the underlying mechanism, an injection spectrum dependent on the jet-power is required to give the observed dependence of spectral index at rest-frame $151 \mathrm{MHz}$ on luminosity; if the dominant effective break frequency were comparable with rest-frame $151 \mathrm{MHz}$ then the drastic depletion of the power reservoir of the source would have caused it to drop below the flux-limit of a typical flux-limited survey.

Possible evidence that the $P-\alpha$ correlation arises in the way modelled in this paper, namely through the steepening of the energy distribution of particles which are ultimately injected into the lobes by radiative losses in the enhanced magnetic fields of the hotspots of sources with more powerful jets, comes from the non-detection of optical synchrotron emission associated with the hotspots of very powerful radio sources. The number of hotspots which emit optical synchrotron radiation is small, for example there is a deficit of optical hotspots in very powerful radio sources such as Cygnus A (Röser, Meisenheimer \& Yates 1996), whereas optical hotspots are detected in low-power radio sources (Meisenheimer et al. 1989). If it is the case, as we have modelled in this paper, that more powerful sources have higher magnetic fields in their hotspots than do less powerful sources, then the absence of optical synchrotron emission from the hotspots of the former arises simply because the spectrum of the hotspot emission is so steep towards this frequency regime. 

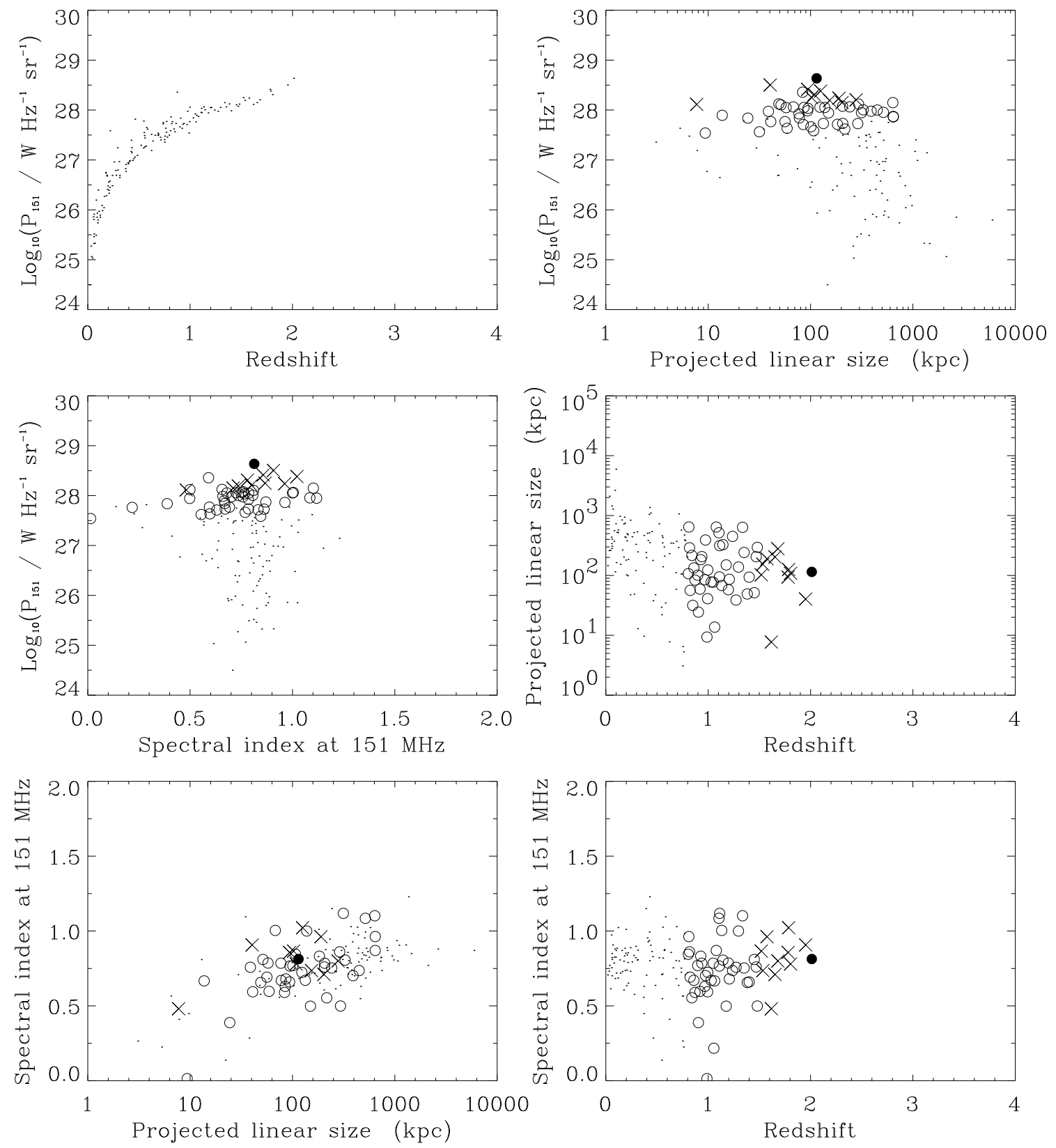

Fig. 16.- Figure showing real 3C data, for FR IIs and DAs, assuming $\Omega_{\mathrm{M}}=1 \& \Omega_{\Lambda}=0$, for the six planes in $[P D z \alpha]$ parameter space. Solid circles indicate objects at $z \geq 2$, crosses indicate objects with $2>z \geq 1.5$, open circles indicate $1.5>z \geq 0.8$ and dots indicate sources with $z<0.8$. 

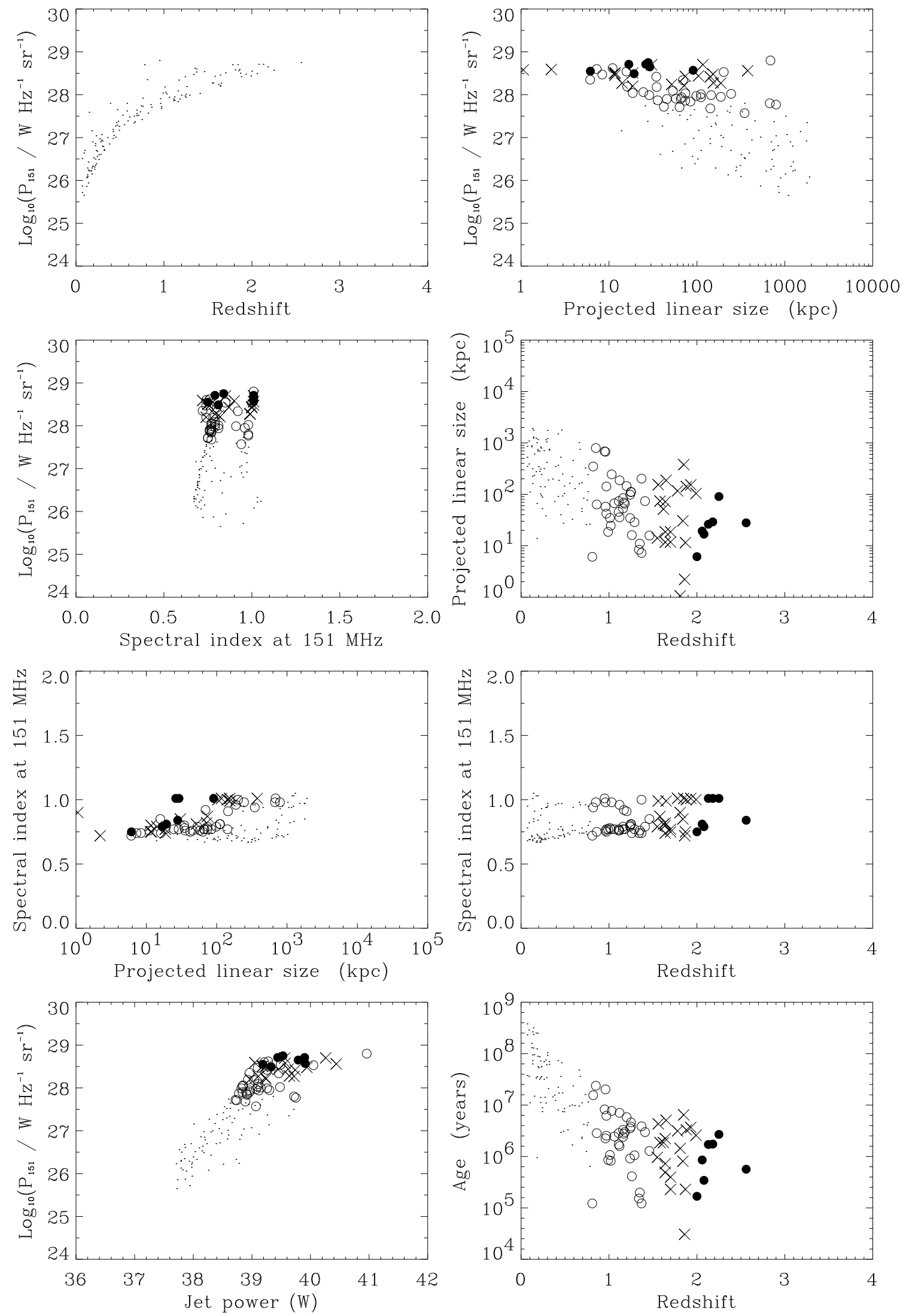

Fig. 17.- Figure showing simulated $3 \mathrm{C}$ data, assuming $\Omega_{\mathrm{M}}=1 \& \Omega_{\Lambda}=0$, for the six planes in $[P D z \alpha]$ parameter space and additionally a plot of the jet-power $Q_{\mathrm{o}}$ vs rest-frame luminosity at $151 \mathrm{MHz}$ and a plot of the age of each source at the point we observe it against redshift. Solid circles indicate objects at $z \geq 2$, crosses indicate objects with $2>z \geq 1.5$, open circles indicate $1.5>z \geq 0.8$ and dots indicate sources with $z<0.8$. 

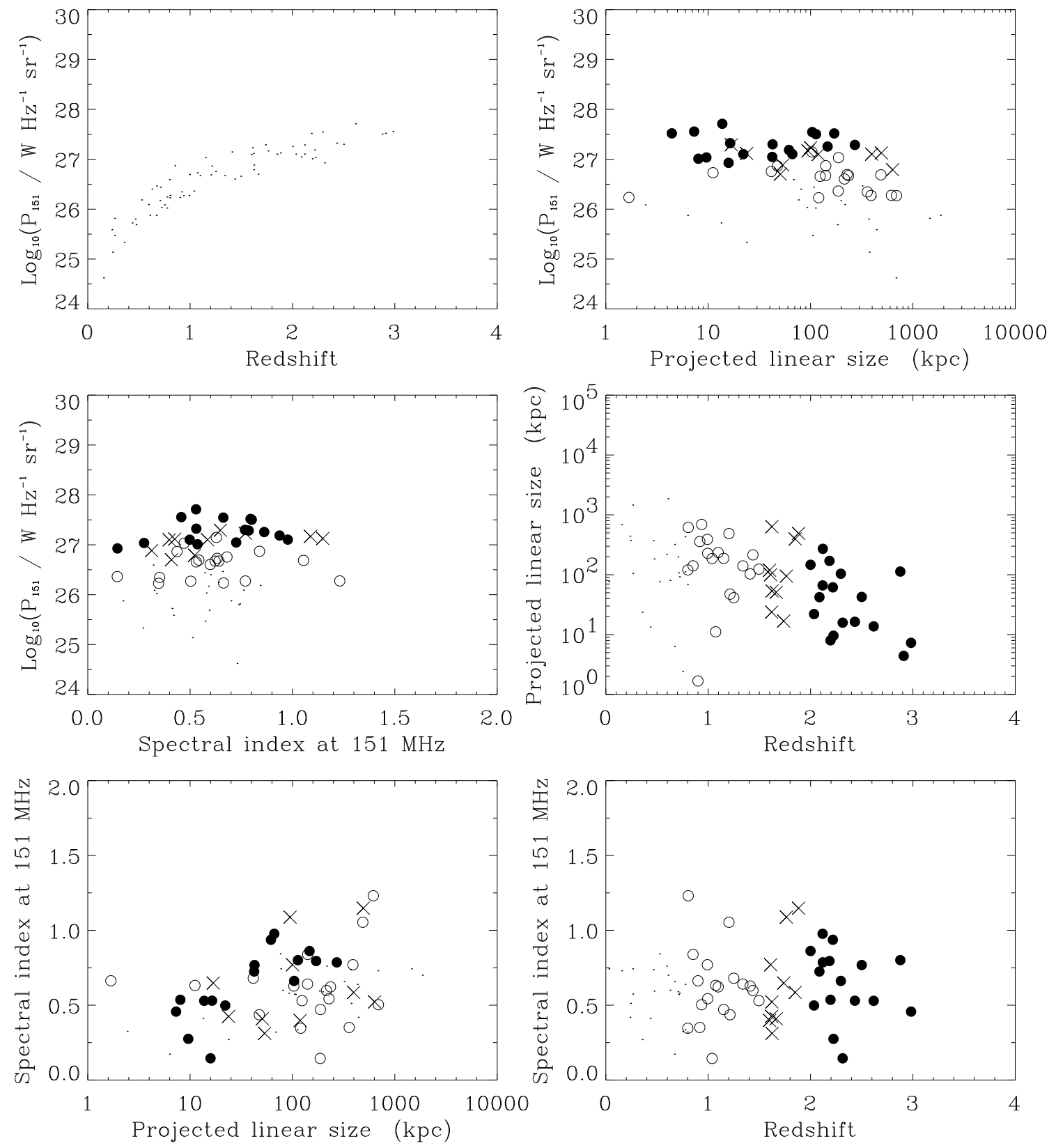

Fig. 18.- Figure showing real 7C data, for FR IIs and DAs, assuming $\Omega_{\mathrm{M}}=1 \& \Omega_{\Lambda}=0$ for the six planes in $[P D z \alpha]$ parameter space. Solid circles indicate objects at $z \geq 2$, crosses indicate objects with $2>z \geq 1.5$, open circles indicate $1.5>z \geq 0.8$ and dots indicate sources with $z<0.8$. 

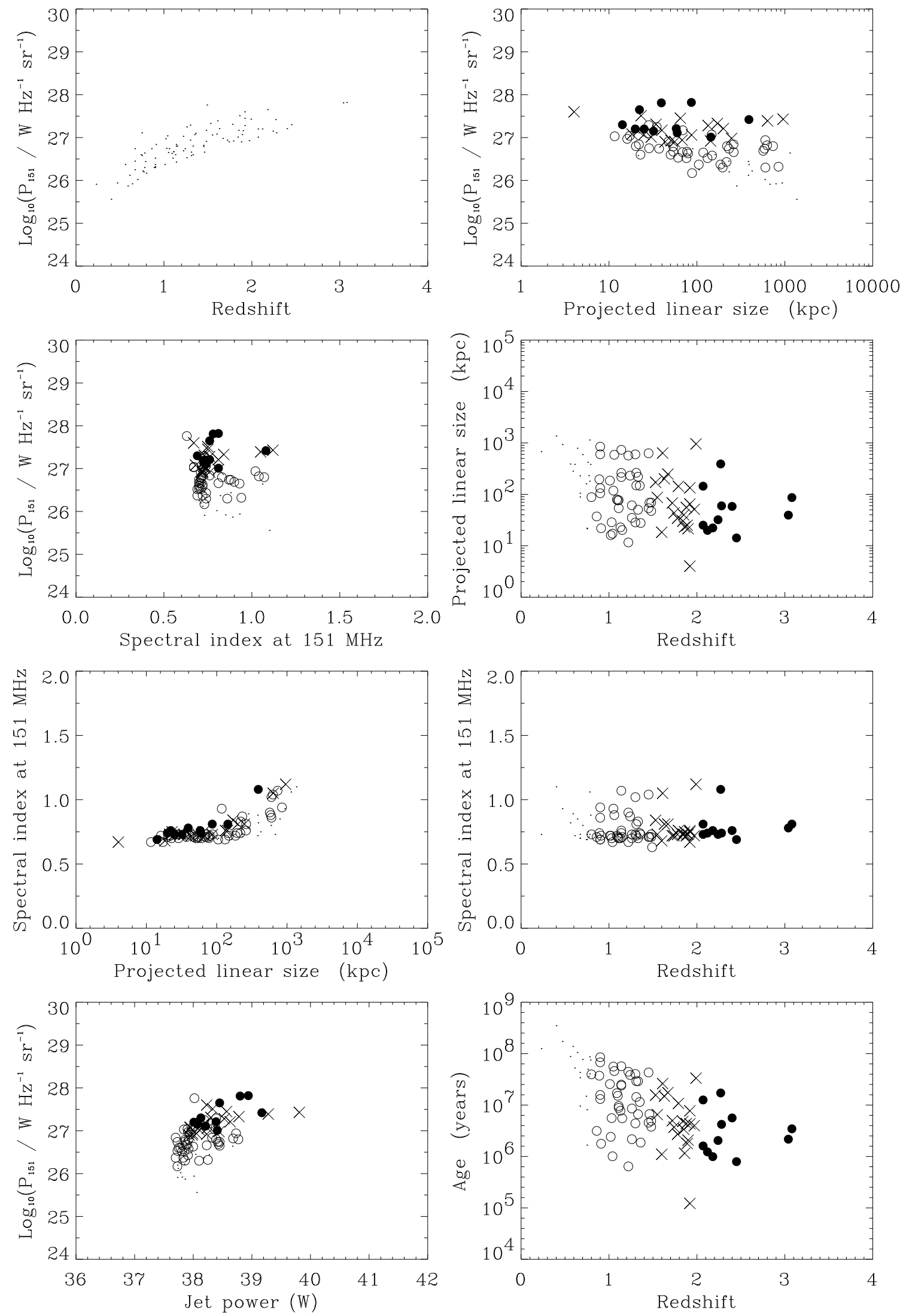

Fig. 19.- Figure showing simulated $7 \mathrm{C}$ data, assuming $\Omega_{\mathrm{M}}=1 \& \Omega_{\Lambda}=0$, for the six planes in $[P D z \alpha]$ parameter space and additionally a plot of the jet-power $Q_{\mathrm{o}}$ vs rest-frame luminosity at $151 \mathrm{MHz}$ and a plot of the age of each source at the point we observe it against redshift. Solid circles indicate objects at $z \geq 2$, crosses indicate objects with $2>z \geq 1.5$, open circles indicate $1.5>z \geq 0.8$ and dots indicate sources with $z<0.8$. 
If the $P-\alpha$ correlation arises in a way resembling that which we have modelled here, then it follows that hotspots with more powerful magnetic fields being fed by jets which transport higher powers, should themselves have steeper spectra than the hotspots in sources with lower jet-powers. Such a finding has been pointed out by Scheuer (1998), that the pairs of hotspots in powerful radio sources have steeper spectra than those in less powerful sources.

Previous work [e.g. Baldwin 1982] has shown that simple models of radio source evolution predict far more high $P$, large $D$ sources than are actually observed. We are able to generate a distribution of points on the $P-D$ plane which does not give rise to such a bulge. This is in part because the higher $Q_{\mathrm{o}}$ sources have steeper spectra and so are more easily lost below the survey flux-limits; this is in addition to the effect whereby the sample selection process itself discriminates against sources which have suffered large adiabatic losses.

We consider the question posed by Jenkins \& Scheuer (1976) 'what docks the tails of radio source components?'. Jenkins \& Scheuer found no evidence that the tails (i.e. bridge towards the core) of radio sources were longer at low-frequency than at highfrequency, as might be expected if synchrotron losses play a major part in limiting the lengths of tails. We contend that a break in the energy spectrum of particles injected into the lobe is an increasingly relevant factor in depleting the bridges of radio sources in increasingly powerful sources. An alternative explanation is that the backflow speeds in these sources are too slow for lobe emission to ever reach the core; a possible test between these two explanations is by consideration of the polarisation patterns in the lobes of these sources (Miller 1985). Inverse Compton losses arising from the AGN's own radiation field will also deplete the lobe emission close into the core (see \$.1.6.

\subsection{The tightness of the $P-z$ correlation}

Application of a flux-limit to a survey means that at the lower luminosity edge there will be a tight correlation between luminosity and redshift. Just how tight the upper edge of the correlation is depends on (i) the steepness and/or curvature of the distribution in jet-powers (see equation 38) (ii) the proximity to the upper-limit of the distribution in jet-powers (note how the $3 \mathrm{C} P-z$ distribution is much tighter than that of the $7 \mathrm{C}$ distribution comparing Figures 16 and 18., (iii) the energy distribution of the particles injected into the lobes as discussed in $\$ 8.4 .2$ and (iv) the birth function itself. The lack of high redshift sources in $3 \mathrm{C}$ may arise in part because of an intrinsic maximum jetpower (= maximum black hole mass) in these objects (as discussed in Willott et al. 1998b).

\subsection{Dependence of spectral index on linear size}

The tendency for radio sources with larger linear sizes to have steeper spectra, as seen in Figure 8, is not hard-wired into our simulations, yet it does appear (e.g. Figure 19). We interpret this tendency as being due to the decrease in magnetic field as the lobes expand. For a fixed observing frequency, the Lorentz factor of particles contributing most of their emission at this frequency will be higher for a lower magnetic field (see equation 3). Thus particles with a higher Lorentz factor are required for the chosen emitting frequency and given the power-law exponent of typical energy distributions, the number of high Lorentz factor particles is smaller than the number of less energetic particles. Moreover, the adiabatic expansion process itself, while preserving the shape of the spectrum, will shift to lower frequencies any features in the spectrum such as a break frequency.

\subsection{Ramifications of the 'youth-redshift link'}

Each of the Figures 17 and 19 show (bottom right hand corners) plots of the ages of sources in the given simulated sample versus the redshifts. There is a very steep (negative) gradient seen in the distribution of points on these plots: objects close to a redshift of 2 have ages approaching two orders of magnitude smaller than objects in the local Universe; the correspondence in the simulated fainter sample (Figure 19) is the same but less pronounced. We thus conclude that in any study of the importance of redshift to a given phenomenon, it is essential to take into account the fact that the higher the redshift at which an object is observed, the younger (on average) it is. Two such issues where this is an important consideration are (i) the higher distortion in radio structure of Barthel and Miley's (1988) high-redshift sample of steep-spectrum quasars compared with their low-redshift sample and (ii) the alignment effect [McCarthy et al. 1987; Chambers, Miley \& van Breugel 1987]. For case (i) Barthel \& Miley suggested that at higher redshifts the environments of radio sources were more dense and inhomogeneous than at low redshift. In case (ii) it has 
been proposed that the origin of the optical and infrared light which is aligned with the radio axes arises either because of star-formation stimulated by the passage of the jet through the medium [McCarthy et al. 1987; Rees 1989] or by quasar light escaping along the radio axes scattered into the line-of-sight by dust or electrons [Barthel 1989; Fabian 1989].

Sources which are younger may have the interactions of their jets occuring much closer in to the host galaxy where there is a higher density and likely greater inhomogeneity in the ambient matter; thus it is possible that star-formation is more easily triggered here than at distances further out from the central engine and possible that the radio structures seen at these times are more distorted than those seen much later in the life of a radio source. Although a young source is not necessarily a short source (consider the derivative of equation (6), in the case of sources which are young but quite long on account of their high jet-power $Q_{\mathrm{o}}$, the higher jet-power may well be more efficient at triggering star formation.

We note Dey et al.'s (1997) spectropolarimetric study of $4 \mathrm{C} 41.17$ presents a compelling case for jettriggered star formation in this young, high jet-power, radio galaxy at $z=3.8$.

Moreover if it is the case that mergers of galaxies trigger radio galaxy or quasar activity (see e.g. Sanders et al. 1988), then observations of young radio sources (and thus those at high redshift) will be revealing the emergence of a radio source through recent merger products. Such an environment is more likely to be favourable to the triggering of star-formation than the rather more 'steady-state' environment of a radio source which has been in existence for longer than $10^{8}$ years.

Consistent with this picture is the finding of Best, Longair and Röttgering (1996) that the smallest sources in their sample of $z \sim 1$ radio galaxies (all with very similar luminosities) are those which are most aligned with optical emission. Best et al. (1996) remarked that the sequence of changing optical aligned structure with increasing radio size could be naturally interpreted by comparing it with different phases of the interaction of the radio jets with the interstellar and intergalactic medium as the radio sources age.

We have not needed to invoke any systematic change in the environments of radio sources with redshift in order to reproduce good approximations to the six planes in $[P D z \alpha]$ space from our observations.
However, we note that Garrington \& Conway (1991) found that there was a tendency for depolarisation to be higher in sources with a higher $P$ (and hence $z$, since their sample mainly comprised objects in the $3 \mathrm{C}$ sample or similar samples). It is possible that this is related to the above discussion: objects in their sample with higher $P$ or $z$ which are younger may well be in much more recently merged environments with the consequence that inhomogeneous regions of higher ambient density will more readily depolarise polarised intensity radiated from the lobes in these environments.

Note that a result of this work is that we are unavoidably driven to the conclusion that the high redshift sources we find in a radio survey are inevitably a younger population than their low redshift counterparts. Comparison of the simulated samples in Figures 17 and 19 suggest that the strong dependence of age on redshift is alleviated significantly by studies using more than one complete sample e.g. the new 7C redshift survey in tandem with the $3 \mathrm{C}$ sample.

Even if some details of our model are not fully correct and for example, the inclusion of a source into a survey is governed much more by its low-frequency emission being dominated by its hotspots than we infer to be the case on the basis of current data, then in order to avoid simulating an excess of very powerful large linear size radio sources over that which is seen, it would be necessary to invoke that sources at higher redshift have their beams switched on for shorter times. The "youth-redshift degeneracy" would then be truely hard-wired into the model. As it is, in our model the "youth-redshift degeneracy" arises because older sources do not survive above the survey fluxlimit because the spectral losses due to (especially) inverse Compton and also adiabatic expansion and synchrotron losses deplete the luminosity of an object at the relevant emitted frequency $[(1+z) \times$ survey frequency].

The model we have presented here, which reproduces the nature of radio sources which are included in low-frequency selected radio surveys, does not need to invoke the 'death' of radio sources. This gives us a distribution in the ages of the sources which make it into our samples but does not require any strong statement about their lifetimes. It has been suggested that radio sources which are on death row should be seen as a population of sources which simply appear as widely separated pairs of "naked hotspots". We suggest that this is unlikely: the inclusion of such 
objects into low-frequency (e.g. $151 \mathrm{MHz}$ ) surveys is most unlikely to be via their hotspots because, as discussed in 8.1 .5 hotspots do not radiate strongly at these low frequencies; although such hotspots will radiate at frequencies like $1.4 \mathrm{GHz}$ [e.g. as in the FIRST survey of Becker et al. (1998)] it is most unlikely that emission will be found to link apparently unrelated isolated hotspots.

\section{Summary of conclusions}

New observational results

The population of radio sources which are detected by a given survey cannot be naively identified with the real population of radio sources out there: our study of a number of complete samples of radio sources selected at frequencies close to $151 \mathrm{MHz}$, with a coverage of the luminosity-redshift plane which is substantially improved over previous studies, has led us to conclude that the principal dependences between properties of these radio sources selected in complete samples are as follows:

1. The rest-frame spectral index at low-frequency depends on the luminosity of a source $(P-\alpha$ correlation).

2. The rest-frame spectral index at high-frequency $(\mathrm{GHz})$ depends on the redshift of a source.

3. Observed-frame spectral indices are also governed by the redshift of a source.

4. Sources with larger physical sizes have steeper spectra ( $D-\alpha$ correlation).

5. Sources at higher redshift have smaller physical sizes $(D-z$ anti-correlation).

New views of radio sources

6. We have presented an intuitive picture for the random interception of radio sources with our light-cone, which does not necessitate the use of assumptions concerning the dependences of the lifetimes of radio-sources.

7. The $P-\alpha$ correlation (result 1 , listed above) can be understood if the hotspot is viewed as the governor of the energy distribution injected into the lobes of radio sources, and if the jetpower determines the magnetic field density in the hotspots.

8. The $D-z$ anti-correlation (result 4 ) arises because of a more fundamental dependence, namely that the high-redshift sources in a given sample will be substantially younger than their low redshift counterparts, an effect which we call the 'youth-redshift degeneracy'. 


\section{Acknowledgments}

We warmly thank collaborators past and present: Dave Rossitter, Steve Eales, Mark Lacy and Julia Riley, for their contributions to the acquisition of data. It is a pleasure to thank Beatriz Bengoechea, Stephen Blundell, Christian Kaiser, Paddy Leahy, John Miller, Lance Miller, Prasenjit Saha, Alan Sanders, Chris Simpson, Devinder Sivia and Graham Woan for useful discussions and/or assistance. This research has made use of the NASA/IPAC Extragalactic Database, which is operated by the Jet Propulsion Laboratory, Caltech, under contract with the National Aeronautics and Space Administration and of the Atlas of $3 \mathrm{CRR}$ radio sources. KMB thanks Balliol College, Oxford for a Research Fellowship.

\section{REFERENCES}

Allington-Smith, J.R., 1982, MNRAS, 199, 611

Allington-Smith, J.R., 1984, MNRAS, 209, 665

Allington-Smith, J.R., Lilly, S.J., \& Longair, M.S., 1985, MNRAS, 213, 243

Allington-Smith, J.R., Spinrad, H., Djorgovski, S., Liebert, J., 1988, MNRAS, 234, 1091

Baars, J.W.M., Genzel, R., Pauliny-Toth, I.I.K., \& Witzel, A., 1977, A\&A, 61, 99

Baldwin, J.E., 1982, in 'Extragalactic Radio Sources' eds Heeschen, D.S., Wade C.M., Reidel, Dordecht, 21

Barthel, P.D. \& Miley, G.K., 1988, Nature, 333, 319

Barthel, P.D., 1989, ApJ, 336, 606

Becker, R.H., Helfand, D.J., White, R.L., Gregg, M.D., Laurent-Muehleisen, S.A., 1998, http://sundog.stsci.edu

Begelman, M.C., Blandford, R.D., \& Rees, M.J., 1984, Rev. Mod. Phys., 56, 280

Begelman, M.C. \& Cioffi, D.F., 1989, ApJ, 345, 21

Bell A.R., 1978, MNRAS, 182, 147

Bennett A.S., 1962, MmRAS, 68, 163

Best, P., Bailer, D.M., Longair, M.S., \& Riley, J.M., 1995, MNRAS, 275, 1171

Best, P.N., Longair, M.S., \& Röttgering, H.J.A., 1996, MNRAS, 280, L9

Best, P.N., Longair, M.S., \& Röttgering, H.J.A., 1998, MNRAS, 295, 549

Black, A.R.S., Baum, S.A., Leahy, J.P., Perley, R.A., Riley, J.M., Scheuer, P.A.G., 1992, MNRAS, 256, 186

Blumenthal, G. \& Miley, G., 1979, A\&A, 80, 13

Blundell, K.M. \& Lacy M., 1995, MNRAS, 274, L9

Blundell, K.M., Rawlings, S., Eales, S.A., Taylor, G.B., \& Bradley, A.D., 1998, MNRAS, 295, 265 [Blundell et al. 1998a] 
Blundell, K.M., Rawlings, S., Riley, J.M., Willott, C.J., \& Laing, R.A., 1998, to be submitted to New Ast. [Blundell et al. 1998b]

Bridle, A.H., Davies, M.M., Fomalont, E.B., Lequeux, J., 1972, AJ, 77, 405

Bridle, A.H., Hough, D.H., Lonsdale, C.J., Burns, J.O., \& Laing, R.A., 1994, AJ, 108, 766

Brunetti, G., Setti, G., \& Comastri, A., 1997, A\&A, 325,898

Buchalter, A., Helfand, D.J., Becker, R.H., \& White, R.L., 1998, ApJ, 494, 503

Carilli, C.L., Perley, R.A., Dreher, J.W., \& Leahy, J.P., 1991, ApJ, 383, 554

Carroll, S.M., Press, W., \& Turner, E.L., 1992, ARA\&A, 30, 499

Chambers, K.C., Miley, G.K., \& van Breugel, W.J.M., 1987, Nature, 329, 604

Chambers, K.C., Miley, G.K., \& van Breugel, W.J.M., 1990, ApJ, 363, 21

Clarke, D.A., Norman, M.L., \& Burns, J.O., 1989, ApJ, 342, 700

Colla, G., Fanti, C., Fanti, R., Ficarra, A., Formiggini, L., Gandolfi, E., Gioia, I., Lari, C., Marano, B., Padrielli, L., Tomasi, P., 1973, AASS, 11,291

Condon J.J., Helou G., Sanders D.B., \& Soifer, B.T., 1990, ApJS, 73, 359

Condon, J.J., Cotton, W.D., Greisen, E.W., Yin, Q.F., Perley, R.A., Taylor, G.B., \& Broderick, J.J., 1998, AJ, 115, 1693 http://www.cv.nrao.edu/ jcondon/nvss.html

Crawford, C.S. \& Fabian, A.C., 1996, MNRAS, 282, 1483

De Young, D.S., 1997, ApJ, 490, L55

Dey, A., van Breugel, W., Vacca, W.D., \& Antonucci, R., 1997, ApJ, 490, 698

Dunlop, J.S., \& Peacock, J.A., 1990, MNRAS, 247, 19

Dunlop, J.S. \& Peacock J.A., 1993, MNRAS, 263, 936
Eales, S.A., 1985, MNRAS, 217, 149

Eales, S.A. \& Rawlings, S., 1993, ApJ, 441, 67

Eales, S.A., Alexander, P., \& Duncan W.D., 1989, MNRAS, 240, 817

Eales, S.A., Rawlings S., Dickinson, M., Spinrad H., Hill, G.J., \& Lacy, M., 1993, ApJ, 409, 578

Eales, S., Rawlings, S., Law-Green, D., Cotter, G., Lacy, M., 1997, MNRAS, 291, 593

Eales, S.A., \& Rawlings, S., 1996, ApJ, 460, 68

Fabian, A.C., 1989, MNRAS, 238, 41P

Falle, S.A.E.G., 1991, MNRAS, 250, 581

Fanaroff, B.L. \& Riley, J.M., 1974, MNRAS, 167, 31P

Ficarra, A., Grueff, G., \& Tomassetti, G., 1985, AASS, 59, 255

Garrington, S.T. \& Conway, R.G., 1991, MNRAS, 250,198

Hales, S.E.G., Baldwin, J.E., \& Warner, P.J., 1988, MNRAS, 234, 919

Hales, S.E.G., Baldwin, J.E., \& Warner, P.J., 1993, MNRAS, 263, 25

Hales, S.E.G., Waldram, E.M., Rees, N., \& Warner, P.J., 1995, MNRAS, 274, 447

Hardcastle, M.J., Alexander, P., Pooley, G.G., Riley, J.M., 1997, MNRAS, 288, 859

Harris, D.A., Leighly, K.M., \& Leahy, J.P., 1998, ApJ, 499, 149

Heavens A.F. \& Drury L.O'C., 1988, MNRAS, 235, 997

Heavens, A.F. \& Meisenheimer K., 1987, MNRAS, 225,335

Heeschen, D.S., 1960, PASP, 72, 368

Hewitt, A. \& Burbidge, G., 1980, ApJS, 43, 57

Hill, G.J. \& Lilly, S.J., 1991, ApJ, 367, 1

Jenkins, C.J. \& Scheuer, P.A.G., 1976, MNRAS, 174, 327 
Kaneda, H., Tashiro, M., Ikebe, Y., Ishisaki, Y., Kubo, H., Makishima, K., Ohashi, T., Saito, Y., Tabara, H. \& Takahashi, T., 1995, ApJ, 453, L13

Kaiser, C.R. \& Alexander, P., 1997, MNRAS, 286, 215

Kaiser, C.R., Dennett-Thorpe J., \& Alexander, P., 1997, MNRAS, 292, 723

Kapahi, V.K., 1987, AJ, 97, 1

Komissarov, S.S. \& Falle, S.A.E.G., 1998, MNRAS, 297, 1087

Krolik, J.H. \& Chen, W., 1991, AJ, 102, 1659

Lacy, M., Hill, G.J., Kaiser M.-E. \& Rawlings, S., 1993, MNRAS, 263, 707

Laing, R.A., Riley, J.M., \& Longair, M.S., 1983, MNRAS, 204, 151

Laing, R.A. \& Peacock, J.A., 1980, MNRAS, 190, 903

Landau, L.D. \& Lifshitz, E.M., 1959, Fluid Mechanics, pg 335, Pergamon Press

Law-Green, J. D. B., Leahy, J. P., Alexander, P., Allington-Smith J. R., van Breugel W.J.M., Eales S.A., Rawlings S.G., \& Spinrad, H., 1995, MNRAS, 274, 939

Leahy, J.P., 1991, in Hughes P.A., ed., Beams and Jets in Astrophysics, pg 119, CUP, Cambridge

Leahy, J.P., Black, A.R.S., Dennett-Thorpe, J., Hardcastle, M.J., Komissarov, S., Perley, R.A., Riley, J.M., \& Scheuer, P.A.G., 1997, MNRAS, 291, 20

Leahy, J.P. \& Perley R.A., 1991, AJ, 102, 537

Leahy, J.P., Muxlow, T.W.B., \& Stephens, P.W., 1989, MNRAS, 239, 401

Leahy, J.P. \& Williams, A.G., 1984, MNRAS, 210, 929

Lilly, S.J., 1989, ApJ, 340, 77

Lilly, S.J. \& Longair, M.S., 1984, MNRAS, 211, 833

Lind, K.R., Payne, D.G., Meier, D.L., \& Blandford, R.D., 1989, ApJ, 344, 89

Liu, R., Pooley, G. \& Riley, J.M., 1992, MNRAS, 257, 545
Longair, M.S. \& Riley, J.M., 1979, MNRAS, 188, 625

Longair M.S., Ryle M. \& Scheuer P.A.G., 1973, MNRAS, 164, 243

Machalski, J. \& Condon, J.J., 1983, AJ, 88, 143

Machalski, J. \& Condon, J.J., 1985, AJ, 90, 973

Macklin, J.T., 1982, MNRAS, 199, 1119

McCarthy, P.J., 1991, AJ, 102, 518

McCarthy, P.J., van Breugel, W..J.M., Spinrad, H., \& Djorgovski, S., 1987, ApJ, 321, L29

McCarthy, P.J., van Breugel, W., Kapahi, V.K., \& Subrahmanya, C.R., 1991, AJ, 102, 522

McGilchrist, M.M., Baldwin, J.E., Riley, J.M., Titterington, D.J., Waldram, E.M., \& Warner, P.J., 1990, MNRAS, 246, 110

Meisenheimer, K., Röser, H.-J., Hiltner, P.R., Yates, M.G., Longair, M.S., Chini, R., \& Perley, R.A., 1989, A\&A, 219, 63

Miley, G., 1980, ARA\&A, 18, 165

Miller, L., 1985, MNRAS, 215, 773

Mulchaey, J.S. \& Zabludoff, A.I., 1998, ApJ, 496, 73

Naundorf, C.E., 1992, M.Phil. Thesis, University of Cambridge

Naundorf C. E., Alexander P., Riley J.M., \& Eales S.A., 1992, MNRAS, 258, 647

Neeser, M.J., Eales, S.A., Law-Green, J.D., Leahy, J.P., \& Rawlings, S., 1995, ApJ, 451, 76

Nilsson, K., Valtonen, M.J., Kotilainen, J., \& Jaakkola, T., 1993, ApJ, 413, 453

Norman M.L., Winkler K.-H.A., Smarr L., \& Smith M.D., 1982, A\&A, 113, 285

O’Dea, C.P., Baum, S.A., \& Stanghellini, C., 1991, ApJ, 380, 66

Oort, M.J.A., Katgert, P., \& Windhorst, R.A., 1987, Nature, 328, 500

Owsianik, I. \& Conway, J.T., 1998, A\&A, 337, 69

Peacock, J.A. \& Wall, J., 1985, MNRAS, 216, 173 
Pearson, T.J. \& Kus, A.J., 1978, MNRAS, 182, 273

Purvis, A., Tappin, S.J., Rees, W.G., Hewish, A., \& Duffett-Smith, P.J., 1987, MNRAS, 229, 589

Rawlings, S., Lacy, M., Blundell, K.M., Eales, S.A., Bunker, A.J., \& Garrington, S.T., 1996, Nature, 383, 502 [Rawlings et al. 1996a]

Rawlings S., Lacy M., Leahy J.P., Dunlop J.S., Garrington S.T., \& Ludke E., 1996, MNRAS, 279, L13 [Rawlings et al. 1996b]

Readhead, A.C.S., Taylor, G.B., Pearson, T.J., \& Wilkinson, P.N., ApJ, 460, 634

Rees, M.J., 1989, MNRAS, 239, 1P

Rees, N., 1990, MNRAS, 244, 233

Riley, J.M., 1989, MNRAS, 238, 1055

Roche, N., Eales, S.A., \& Rawlings, S., 1998, MNRAS, 297, 405

Röser, H.-J., Meisenheimer, K. \& Yates, M., 1996, in 'Cygnus A - Study of a Radio Galaxy', eds C.L. Carilli and D.E. Harris, p121, C.U.P.

Rossitter, D., 1987, Ph.D. Thesis, University of Cambridge

Sanders, D.B., Soifer, B.T., Elias, J.H., Madore, B.F., Matthews, K., Neugebauer, G., \& Scoville, N.Z., 1988, ApJ, 325, 74

Scheuer, P.A.G., 1982, in 'Extragalactic Radio Sources' eds Heeschen, D.S., Wade C.M., Reidel, Dordecht, 21

Scheuer, P.A.G., 1987, in Superluminal Radio Sources, ed. J.A. Zensus \& T.J. Pearson

Scheuer, P.A.G., 1995, MNRAS, 277, 331

Scheuer, P.A.G., 1998, priv. comm.

Scheuer, P.A.G. \& Williams, P.J.S., 1968, ARA\&A, 6,321

Simpson, C., Rawlings, S., \& Lacy, M., 1998, submitted to MNRAS

Singal, A.K., 1993, MNRAS, 263, 139

Sivia, D.S., 1996, Data Analysis: a Bayesian Tutorial, p 22, OUP.
Tansley, D., 1997, M.Sc. Thesis, University of Manchester

Taylor, G.B., \& Perley, R.A., 1992, A\&A, 262, 417

Ubachukwu, A.A., 1995, Ap\&SS, 228, 195

Vermeulen R.C. \& Cohen M.H., 1994, ApJ, 430, 467

Vigotti, M., Grueff, G., Perley, R., Clark, B.G., Bridle, A.H., 1989, AJ, 98, 419

Wardle, J.F.C. \& Aaron, S.E., 1997, MNRAS, 286, 425

Weiler, K.W. \& Johnson, K.J., 1980, MNRAS, 190, 269

Willott, C.J., Rawlings, S., Blundell, K.M., \& Lacy, M., 1998a, MNRAS, 300, 625

Willott, C.J., Rawlings, S., Blundell, K.M., \& Lacy, M., 1998b, submitted to MNRAS

Windhorst, R.A., van Heerde, G.M., \& Katgert, P., 1984, AASS, 58, 1

Williams. A., 1991, in Hughes P.A., ed., Beams and Jets in Astrophysics, CUP, pg 356, Cambridge

Yates, M.G., Miller, L. \& Peacock, J.A., 1989, MNRAS, 240, 129

Xu, W., Lawrence, C.R., Readhead, A.C.S., Pearson, T.J., 1994, AJ, 108, 395

This 2-column preprint was prepared with the AAS LATEX macros $\mathrm{v} 4.0$. 
Table 1: This table lists the names, redshifts, projected linear sizes, flux densities at $178 \mathrm{MHz}$ on the scale of Baars et al. (1977)] and at $38 \mathrm{MHz}$ [Hales et al. (1995) and spectral indices between 38 and 178 $\mathrm{MHz}$ of those sources which would not have been selected were the sample criteria to have been $\delta>60^{\circ}$ and $S_{38}>25$, where $S_{38}$ is the flux density in Jy at $38 \mathrm{MHz}$.

\begin{tabular}{llrrrr}
\hline \hline Name & $z$ & $\begin{array}{c}\text { Size } \\
(\mathrm{kpc})\end{array}$ & $\begin{array}{r}S_{178} \\
(\mathrm{Jy})\end{array}$ & $\begin{array}{c}S_{38} \\
(\mathrm{Jy})\end{array}$ & $\alpha_{38}^{178}$ \\
\hline & & & & & \\
3C 268.3 & 0.371 & 9 & 11.7 & 12.3 & 0.0 \\
3C 343 & 0.998 & 9 & 13.5 & 6.3 & -0.5 \\
3C 343.1 & 0.75 & 3 & 12.5 & 9.4 & -0.2 \\
\hline \hline
\end{tabular}


Table 2: Table of radio sources from the sample of Allington-Smith (1982) selected at $408 \mathrm{MHz}$ in a region of sky exactly overlapping with part of the $151-\mathrm{MHz} 6 \mathrm{C}$ sample ( $\$ 3.2$ ) but not common to the $6 \mathrm{C}$ sample. [Objects which would be too bright at $151 \mathrm{MHz}$ to lie within the flux-limits of the $6 \mathrm{C}$ sample are not listed here.] We list their names, redshifts, projected linear sizes in kpc, their flux densities at $151 \mathrm{MHz}$ and $408 \mathrm{MHz}$ and their spectral indices between $151 \mathrm{MHz}$ and $408 \mathrm{MHz}$. Those sources below the horizontal rule are those whose flux densities at $408 \mathrm{MHz}$ fall below $1.1 \mathrm{Jy}$; this flux-limit corresponds to those sources with a flux density of $2 \mathrm{Jy}$ at $151 \mathrm{MHz}$ and a spectral index of 0.6. The angular sizes of B3 0822+39, B2 0927+35, B3 1056+39, B3 1106+37, B2 1129+37, B3 1207+38 and B2 1225+36 were taken from Law-Green et al. (1995); the angular size of B3 1101+38 was taken from the NVSS survey (Condon et al. 1998) and all the remainder were taken from the FIRST survey (Becker et al. 1998). The redshifts indicated by a $\ddagger$ are quoted from Allington-Smith et al. (1988). The redshifts marked by $* \dagger$ are quoted from Allington-Smith, Lilly \& Longair (1985). The redshifts for sources marked with a single asterisk have been measured by S. Rawlings. The redshifts marked with two asterisks are quoted from Eales \& Rawlings (1996); note that the redshift of B2 1132+37 is highly uncertain. The redshift of B31101+38 is quoted from Weiler \& Johnson (1980). The redshift of B2 1204+34 is quoted from Allington-Smith (1984). The redshift of B2 1225+36 is quoted from Xu et al. (1994). The redshift of B2 0927+35 was not available to us and so we considered two limiting values for this. The $151 \mathrm{MHz}$ flux densities of all these objects were taken from the $6 \mathrm{C}$ survey of Hales et al. (1988) and Hales et al. (1993) which are complete to $\gtrsim 0.2 \mathrm{Jy}$. For those sources whose names begin with B2, their flux densities at $408 \mathrm{MHz}$ are taken from the B2.3 catalogue (Colla et al. 1973) and for those sources whose names begin with B3, their flux densities at $408 \mathrm{MHz}$ are taken from the B3 catalogue (Ficarra et al. 1985).

\begin{tabular}{|c|c|c|c|c|c|}
\hline Name & $z$ & $\begin{array}{c}\text { Size } \\
(\mathrm{kpc})\end{array}$ & $\begin{array}{l}S_{151} \\
(\mathrm{Jy})\end{array}$ & $\begin{array}{l}S_{408} \\
(\mathrm{Jy})\end{array}$ & $\alpha_{151}^{408}$ \\
\hline B3 $0822+39$ & $1.2^{*}$ & 8.6 & 0.50 & 1.76 & -1.3 \\
\hline B2 $0835+37$ & $0.396^{\ddagger}$ & 32.0 & 1.62 & 1.26 & 0.3 \\
\hline B2 $0927+35$ & $\left\{\begin{array}{l}0.100 \\
1.000\end{array}\right\}$ & $\left\{\begin{array}{l}11.1 \\
38.3\end{array}\right.$ & 1.70 & 1.15 & 0.4 \\
\hline B3 $1049+38$ & $1.018^{f}$ & 8.5 & 0.99 & 1.24 & -0.2 \\
\hline B3 $1101+38$ & 0.030 & 41.4 & 1.70 & 1.14 & 0.4 \\
\hline B3 $1106+37$ & $2.290^{* *}$ & 5.6 & 1.70 & 1.29 & 0.3 \\
\hline B2 $1129+37$ & $1.060^{*}$ & 162.7 & 1.30 & 1.20 & 0.1 \\
\hline B2 $1132+37$ & $2.880^{* *}$ & 7.4 & 1.10 & 1.52 & -0.3 \\
\hline B3 $1201+39$ & $0.445^{* \dagger}$ & 6.8 & 1.90 & 1.11 & 0.5 \\
\hline B3 $1056+39$ & $2.200^{*}$ & 96.2 & 1.70 & 0.82 & 0.7 \\
\hline B2 1204+34 & 0.11 & 105.0 & 1.97 & 1.01 & 0.7 \\
\hline B3 $1207+38$ & $0.790^{* \dagger}$ & 221.6 & 1.90 & 0.94 & 0.7 \\
\hline B2 $1225+36$ & 1.975 & 0.6 & 0.50 & 1.03 & 0.7 \\
\hline B2 $1245+34$ & $0.409^{\ddagger}$ & 39.0 & 1.80 & 1.06 & 0.5 \\
\hline
\end{tabular}


Table 3: The results of the statistical analysis, run for three different sets of cosmological parameters, on all members of the complete samples used in the analyses.

FR IIs and DAs $\quad \Omega_{\mathrm{M}}=0 \& \Omega_{\Lambda}=0$

\begin{tabular}{|c|c|c|c|}
\hline & $P$ & $D$ & $z$ \\
\hline$\alpha$ & $\begin{aligned} r_{P \alpha \mid z D} & =0.34 \\
\sigma & =5.83\end{aligned}$ & $\begin{aligned} r_{D \alpha \mid P z} & =0.48 \\
\sigma & =8.59\end{aligned}$ & $\begin{aligned} r_{z \alpha \mid P D} & =-0.32 \\
\sigma & =-5.34\end{aligned}$ \\
\hline$z$ & $\begin{array}{r}r_{P z \mid \alpha D}=0.75 \\
\sigma=16.01\end{array}$ & $\begin{aligned} r_{D z \mid P \alpha} & =-0.06 \\
\sigma & =-1.01\end{aligned}$ & \\
\hline $\bar{D}$ & $\begin{aligned} r_{P D \mid z \alpha} & =-0.13 \\
\sigma & =-2.09\end{aligned}$ & & \\
\hline
\end{tabular}

FR IIs and DAs $\quad \Omega_{\mathrm{M}}=1 \& \Omega_{\Lambda}=0$

\begin{tabular}{|c|c|c|c|}
\hline & $P$ & $D$ & $z$ \\
\hline$\alpha$ & $\begin{aligned} r_{P \alpha \mid z D} & =0.34 \\
\sigma & =5.75\end{aligned}$ & $\begin{aligned} r_{D \alpha \mid P z} & =0.48 \\
\sigma & =8.58\end{aligned}$ & $\begin{aligned} r_{z \alpha \mid P D} & =-0.24 \\
\sigma & =-4.05\end{aligned}$ \\
\hline$z$ & $\begin{array}{r}r_{P z \mid \alpha D}=0.65 \\
\sigma=12.62\end{array}$ & $\begin{aligned} r_{D z \mid P \alpha} & =-0.19 \\
\sigma & =-3.06\end{aligned}$ & \\
\hline$D$ & $\begin{aligned} r_{P D \mid z \alpha} & =-0.13 \\
\sigma & =-2.09\end{aligned}$ & & \\
\hline
\end{tabular}

FR IIs and DAs $\quad \Omega_{\mathrm{M}}=\mathbf{0 . 1} \& \boldsymbol{\Omega}_{\Lambda}=\mathbf{0 . 9}$

\begin{tabular}{|c|c|c|c|}
\hline & $P$ & $\bar{D}$ & $z$ \\
\hline$\alpha$ & $\begin{aligned} r_{P \alpha \mid z D} & =0.33 \\
\sigma & =5.65\end{aligned}$ & $\begin{aligned} r_{D \alpha \mid P z} & =0.48 \\
\sigma & =8.46\end{aligned}$ & $\begin{aligned} r_{z \alpha \mid P D} & =-0.32 \\
\sigma & =-5.40\end{aligned}$ \\
\hline$z$ & $\begin{array}{r}r_{P z \mid \alpha D}=0.76 \\
\sigma=16.24\end{array}$ & $\begin{aligned} r_{D z \mid P \alpha} & =-0.05 \\
\sigma & =-0.85\end{aligned}$ & \\
\hline$D$ & $\begin{aligned} r_{P D \mid z \alpha} & =-0.12 \\
\sigma & =-1.88\end{aligned}$ & & \\
\hline
\end{tabular}


Table 4: The results of the statistical analysis, run for three different sets of cosmological parameters, on just the sources with certain FR II structure from the complete samples.

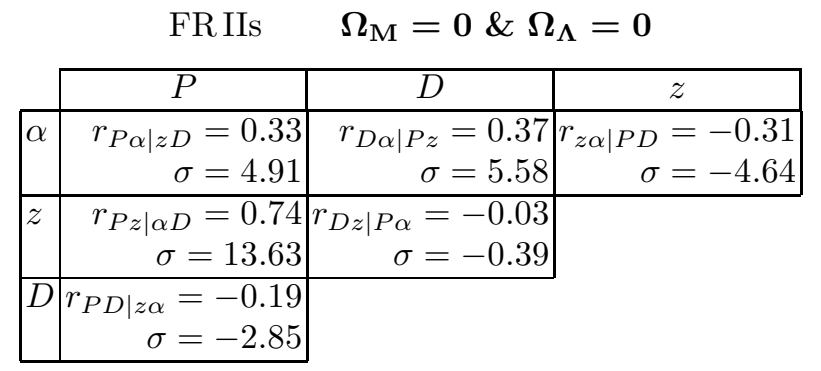

\begin{tabular}{|c|c|c|c|}
\hline \multicolumn{2}{|r|}{ FR IIs } & \multicolumn{2}{|c|}{$\Omega_{M}=1 \& \Omega_{\Lambda}=0$} \\
\hline & $P$ & $D$ & $z$ \\
\hline$\alpha$ & $\begin{aligned} r_{P \alpha \mid z D} & =0.32 \\
\sigma & =4.86\end{aligned}$ & $\begin{aligned} r_{D \alpha \mid P z} & =0.37 \\
\sigma & =5.57\end{aligned}$ & $\begin{aligned} r_{z \alpha \mid P D} & =-0.24 \\
\sigma & =-3.59\end{aligned}$ \\
\hline$z$ & $\begin{array}{r}r_{P z \mid \alpha D}=0.61 \\
\sigma=10.32\end{array}$ & $\begin{aligned} r_{D z \mid P \alpha} & =-0.18 \\
\sigma & =-2.57\end{aligned}$ & \\
\hline $\bar{D}$ & $\begin{aligned} r_{P D \mid z \alpha} & =-0.19 \\
\sigma & =-2.81\end{aligned}$ & & \\
\hline
\end{tabular}

FR IIs $\quad \Omega_{\mathrm{M}}=0.1 \& \Omega_{\Lambda}=0.9$

\begin{tabular}{|c|c|c|c|}
\hline & $P$ & $D$ & $z$ \\
\hline$\alpha$ & $\begin{aligned} r_{P \alpha \mid z D} & =0.32 \\
\sigma & =4.75\end{aligned}$ & $\begin{aligned} r_{D \alpha \mid P z} & =0.36 \\
\sigma & =5.44\end{aligned}$ & $\begin{aligned} r_{z \alpha \mid P D} & =-0.31 \\
\sigma & =-4.67\end{aligned}$ \\
\hline$z$ & $\begin{array}{r}r_{P z \mid \alpha D}=0.74 \\
\sigma=13.87 \\
\end{array}$ & $\begin{aligned} r_{D z \mid P \alpha} & =-0.02 \\
\sigma & =-0.25\end{aligned}$ & \\
\hline$D$ & $\begin{aligned} r_{P D \mid z \alpha} & =-0.18 \\
\sigma & =-2.63\end{aligned}$ & & \\
\hline
\end{tabular}


Table 5: The results of the statistical analysis, run for three different sets of cosmological parameters, on the 3C sample alone.

\begin{tabular}{|c|c|c|c|}
\hline \multicolumn{4}{|c|}{$\Omega_{\mathrm{M}}=0 \& \Omega_{\Lambda}=0$} \\
\hline & $\bar{P}$ & $\bar{D}$ & $z$ \\
\hline$\alpha$ & $\begin{aligned} r_{P \alpha \mid z D} & =0.02 \\
\sigma & =0.20\end{aligned}$ & $\begin{aligned} r_{D \alpha \mid P z} & =0.56 \\
\sigma & =7.42\end{aligned}$ & $\begin{aligned} r_{z \alpha \mid P D} & =0.01 \\
\sigma & =0.12\end{aligned}$ \\
\hline$z$ & $\begin{array}{r}r_{P z \mid \alpha D}=0.97 \\
\sigma=25.24 \\
\end{array}$ & $\begin{aligned} r_{D z \mid P \alpha} & =-0.05 \\
\sigma & =-0.63\end{aligned}$ & \\
\hline$D$ & $\begin{aligned} r_{P D \mid z \alpha} & =-0.03 \\
\sigma & =-0.38\end{aligned}$ & & \\
\hline
\end{tabular}

\begin{tabular}{|c|c|c|c|}
\hline \multicolumn{4}{|c|}{$\Omega_{\mathrm{M}}=1 \& \Omega_{\Lambda}=0$} \\
\hline & $P$ & $D$ & $z$ \\
\hline$\alpha$ & $\begin{aligned} r_{P \alpha \mid z D} & =0.02 \\
\sigma & =0.28\end{aligned}$ & $\begin{aligned} r_{D \alpha \mid P z} & =0.56 \\
\sigma & =7.42\end{aligned}$ & $\begin{aligned} r_{z \alpha \mid P D} & =0.03 \\
\sigma & =0.33\end{aligned}$ \\
\hline$z$ & $\begin{array}{r}r_{P z \mid \alpha D}=0.96 \\
\sigma=22.77 \\
\end{array}$ & $\begin{aligned} r_{D z \mid P \alpha} & =-0.12 \\
\sigma & =-1.39\end{aligned}$ & \\
\hline $\bar{D}$ & $\begin{aligned} r_{P D \mid z \alpha} & =-0.02 \\
\sigma & =-0.20\end{aligned}$ & & \\
\hline
\end{tabular}

\begin{tabular}{|c|c|c|c|}
\hline \multicolumn{4}{|c|}{$\Omega_{\mathrm{M}}=0.1 \& \Omega_{\Lambda}=0.9$} \\
\hline & $P$ & $D$ & $z$ \\
\hline$\alpha$ & $\begin{aligned} r_{P \alpha \mid z D} & =0.03 \\
\sigma & =0.35\end{aligned}$ & $\begin{aligned} r_{D \alpha \mid P z} & =0.55 \\
\sigma & =7.32\end{aligned}$ & $\begin{aligned} r_{z \alpha \mid P D} & =-0.01 \\
\sigma & =-0.13\end{aligned}$ \\
\hline$z$ & $\begin{array}{r}r_{P z \mid \alpha D}=0.98 \\
\sigma=26.20\end{array}$ & $\begin{aligned} r_{D z \mid P \alpha} & =-0.03 \\
\sigma & =-0.33\end{aligned}$ & \\
\hline $\bar{D}$ & $\begin{aligned} & r_{P D \mid z \alpha}=-0.04 \\
& \sigma=-0.47 \\
&\end{aligned}$ & & \\
\hline
\end{tabular}


Table 6: The parameters $n$ and $m$ from equation 1 which have been fitted to the data for the three different assumed cosmologies.

$$
\begin{gathered}
\Omega_{\mathrm{M}}=0 \& \Omega_{\Lambda}=0 \\
\text { FRIIs and DAs }
\end{gathered}
$$

$$
\begin{aligned}
& n[r d z \mid p a]=0.40 \pm 0.30 \\
& n[r d z \mid p]=1.52 \pm 0.30 \\
& n[r d z \mid a]=0.86 \pm 0.20 \\
& m[r p d \mid z a]=0.02 \pm 0.15 \\
& m[r p d \mid z]=0.01 \pm 0.20 \\
& m[r p d \mid a]=0.07 \pm 0.20 \\
& \mathbf{\Omega}_{\mathbf{M}}=\mathbf{1} \& \mathbf{\Omega}_{\boldsymbol{\Lambda}}=\mathbf{0} \\
& \text { FRIIs and DAs }
\end{aligned}
$$

$$
\begin{aligned}
n[r d z \mid p a] & =0.96 \pm 0.25 \\
n[r d z \mid p] & =1.87 \pm 0.30 \\
n[r d z \mid a] & =1.31 \pm 0.20 \\
m[r p d \mid z a] & =0.01 \pm 0.15 \\
m[r p d \mid z] & =0.09 \pm 0.30 \\
m[r p d \mid a] & =0.26 \pm 0.20
\end{aligned}
$$

\section{$\Omega_{\mathrm{M}}=0.1 \& \Omega_{\Lambda}=0.9$ \\ FRIIs and DAs}

$$
\begin{aligned}
n[r d z \mid p a] & =0.35 \pm 0.30 \\
n[r d z \mid p] & =1.41 \pm 0.35 \\
n[r d z \mid a] & =0.76 \pm 0.20 \\
m[r p d \mid z a] & =0.02 \pm 0.20 \\
m[r p d \mid z] & =0.07 \pm 0.30 \\
m[r p d \mid a] & =0.18 \pm 0.20
\end{aligned}
$$

